\title{
IDEOLOGICAL AND THREAT-BASED PREDICTORS OF CYBER VIOLENCE AGAINST WOMEN AND GIRLS
}

\author{
by \\ Arvin Jagayat \\ Bachelor of Science, York University, 2014
}

\begin{abstract}
A thesis
presented to Ryerson University
\end{abstract}

in partial fulfillment of the requirements for the degree of Master of Arts in the program of Psychology

Toronto, Ontario, Canada, 2017 (C) Arvin Jagayat 2017 


\section{AUTHOR'S DECLARATION FOR ELECTRONIC SUBMISSION OF A THESIS}

I hereby declare that I am the sole author of this thesis. This is a true copy of the thesis, including any required final revisions, as accepted by my examiners.

I authorize Ryerson University to lend this thesis to other institutions or individuals for the purpose of scholarly research.

I further authorize Ryerson University to reproduce this thesis by photocopying or by other means, in total or in part, at the request of other institutions or individuals for the purpose of scholarly research.

I understand that my thesis may be made electronically available to the public. 
Ideological and Threat-based Predictors of Cyber Violence Against Women and Girls

Master of Arts, 2017

Arvin Jagayat

Psychology

Ryerson University

\begin{abstract}
Approximately $11 \%$ of women have received some form of unwanted or offensive sexually explicit e-mails, text messages or advances on social networking sites - examples of the many gendered forms of cyber-aggression that constitute cyber violence against women and girls (cyber VAWG). The present thesis developed a cyber VAWG scale and examined sociopolitical ideologies, perceived threats, and ambivalent sexist attitudes as predictors of endorsement of and engagement in cyber VAWG. Study 1 was administered to a university sample of male gamers $(n=46)$, and Study $2(n=276)$ and Study $3(n=6381)$ recruited participants from online video gaming communities. In all three studies, exploratory factor analyses suggested cyber VAWG is a unidimensional psychological construct. Further, path analysis consistently showed that greater hostile sexism predicted greater endorsement, endorsement predicted greater engagement, and greater SDO predicted greater endorsement and engagement. Implications for future research are discussed.
\end{abstract}




\section{Acknowledgements}

I would like to foremost thank both of my parents for everything they've done throughout my life that has allowed me to reach a point where I can undertake graduate studies and be successful in doing so. From the sacrifices they have made to ensure I succeed to their unwavering support of my academic pursuits, I am lucky to have such great role models in my life, and am eternally grateful for the opportunities and unconditional support they continue to give me. The other great role model I would like to thank is my supervisor, Dr. Becky Choma, who has similarly, and constantly, provided me with invaluable opportunities, insight, support, and guidance that have been instrumental in me reaching this milestone in my life. To my role models, to my family, to my friends who have always been there for me, and to all of my peers and colleagues, you have all played a significant part in helping me reach my goals in some form or another over the past two years, and I am forever indebted to you all.

This research was supported by the Social Sciences and Humanities Research Council of Canada. 


\section{Table of Contents}

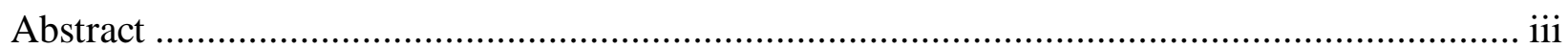

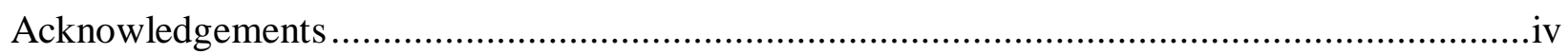

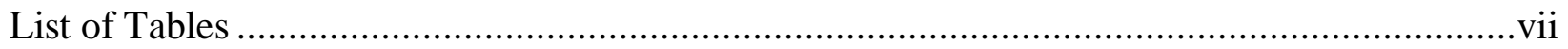

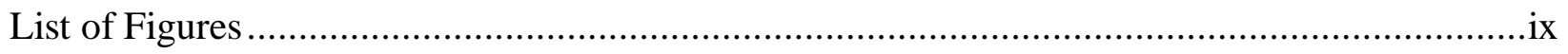

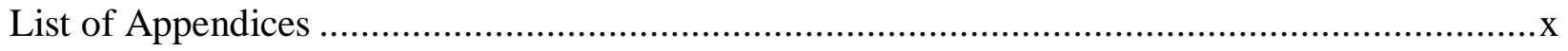

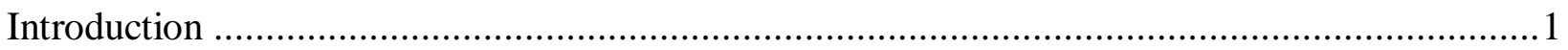

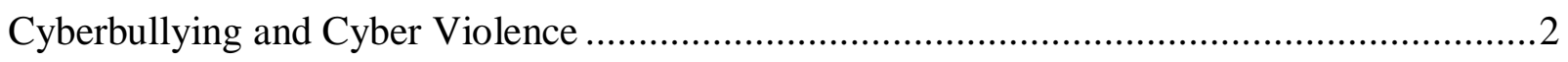

The Role of Gender in Cyber-Aggression ........................................................................6

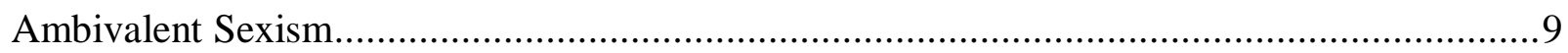

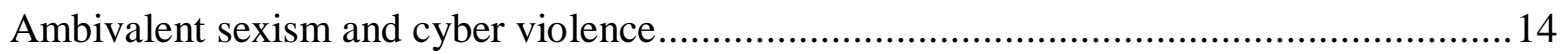

Sociopolitical Ideology: Social Dominance Orientation and Right-Wing Authoritarianism ....15

Perceived Threat and Intergroup Attitudes ………………..............................................26

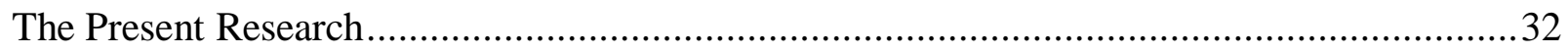

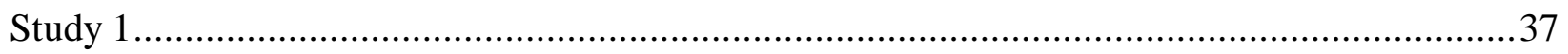

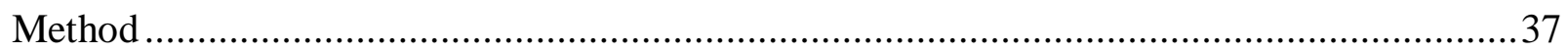

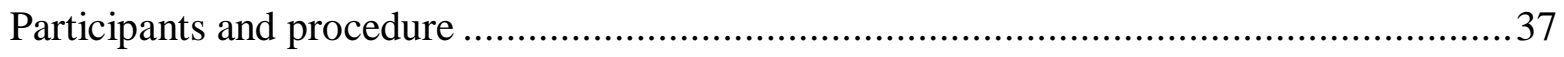

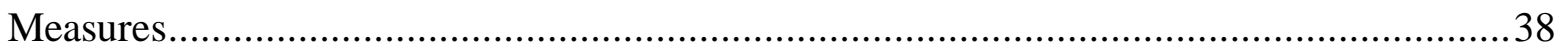

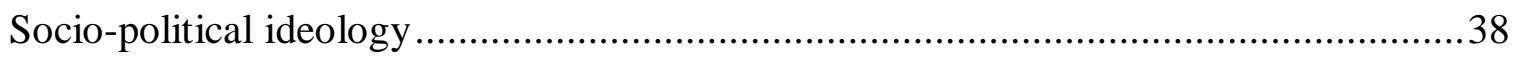

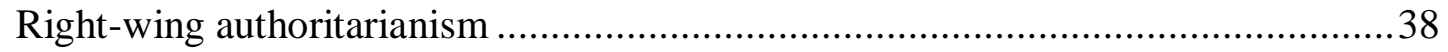

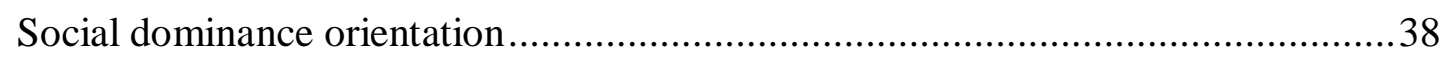

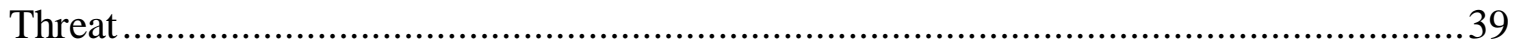

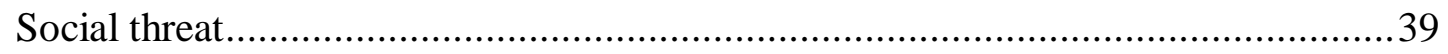




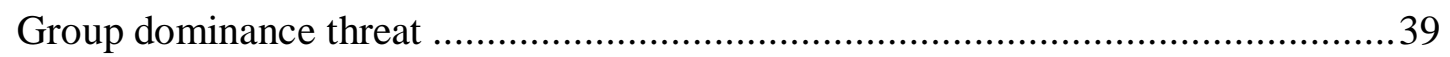

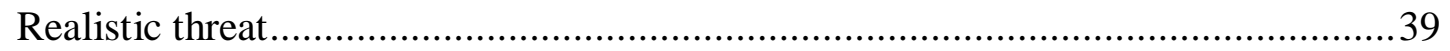

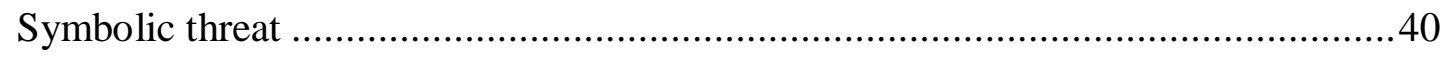

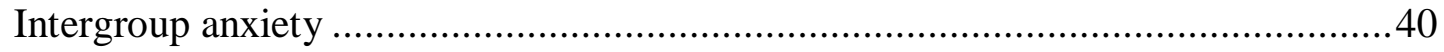

Group esteem threat .................................................................................. 40

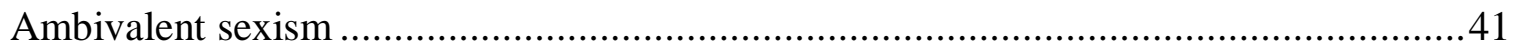

Cyber violence against women and girls ..................................................... 41

Endorsement of cyber violence against women and girls .................................41

Engagement in cyber violence against women and girls ................................42

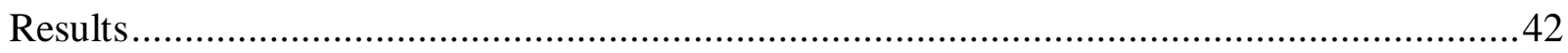

Cyber VAWG endorsement scale development ............................................... 42

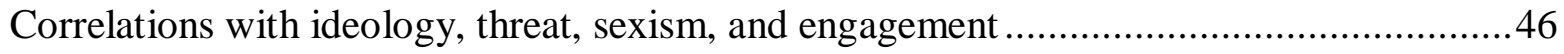

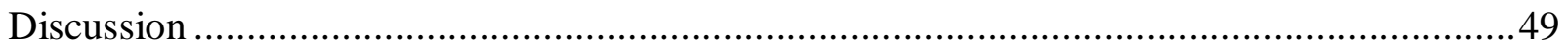

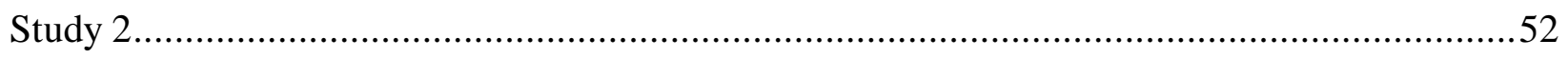

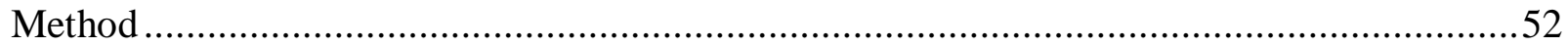

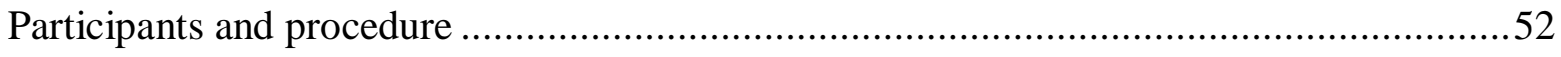

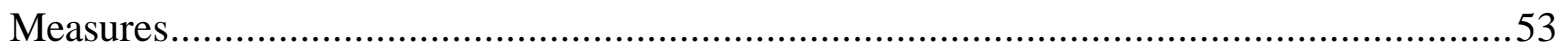

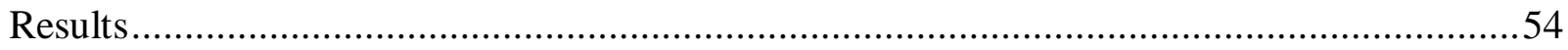

Cyber VAWG endorsement scale development .................................................54

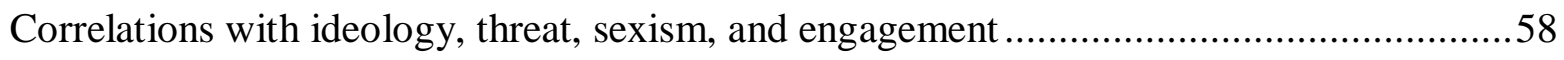

Testing the proposed two-stage mediation model .............................................61

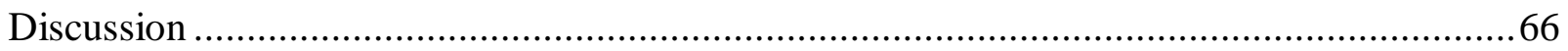




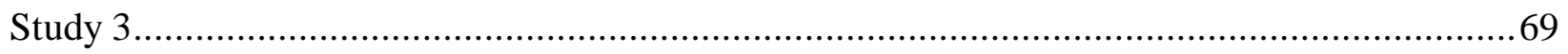

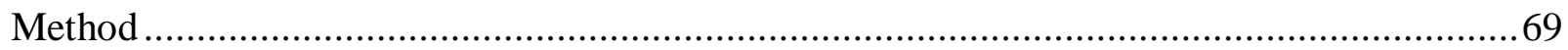

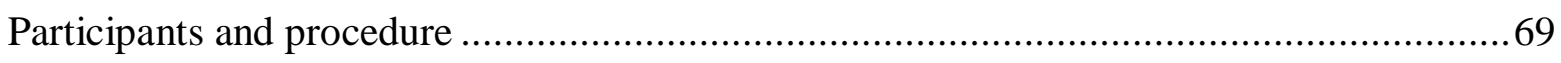

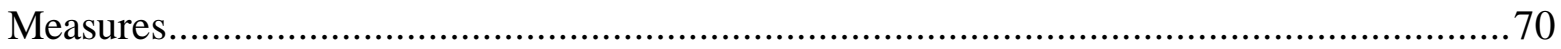

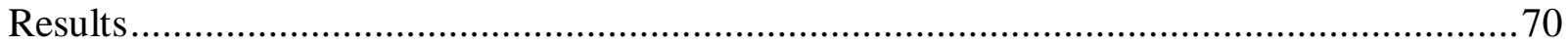

Cyber VAWG endorsement scale development ................................................ 70

Correlations with ideology, threat, sexism, and engagement .................................... 73

Testing the proposed two-stage mediation model …............................................... 76

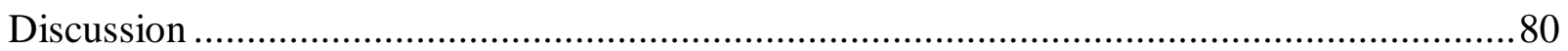

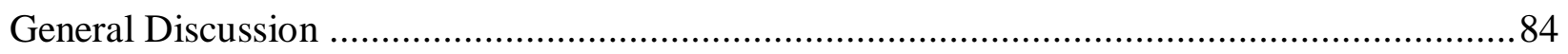

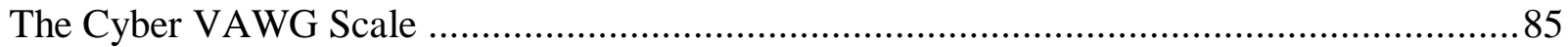

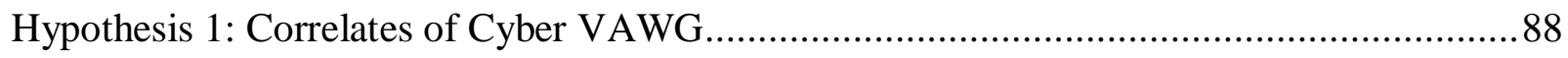

Hypothesis 2: Relations between Ideology with Threat and Sexist Attitudes .......................90

Hypothesis 3: DPM-inspired and ITT-inspired Mediation Models ..................................93

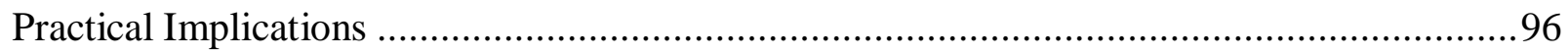

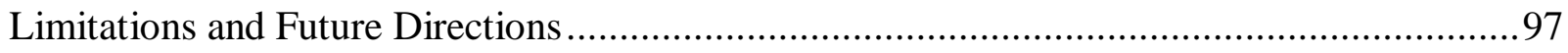

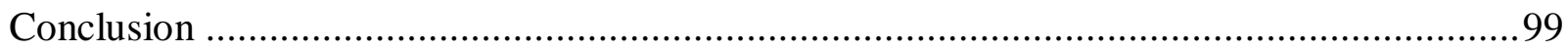

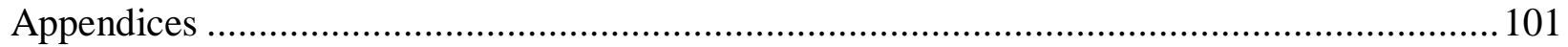

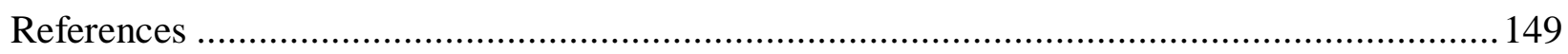




\section{List of Tables}

Table 1.1: Loadings of cyber VAWG items on the single forced factor in Study 1

Table 1.2: Means, standard deviations, skewness, kurtosis, minimum, and maximum values of

Study 1 variables

Table 1.3: Zero-order correlations between Study 1 variables

Table 2.1: Loadings of cyber VAWG items on the single forced factor in Study 2.

Table 2.2: Means, standard deviations, skewness, kurtosis, minimum, and maximum values of

Study 2 variables.

Table 2.3: Zero-order correlations between Study 2 variables.

Table 2.4: Standardized direct and indirect effects of ideology, DPM threats, and sexist attitudes

on endorsement of and engagement in cyber VAWG in Study 2.

Table 2.5: Standardized direct and indirect effects of ideology, non-DPM threats, and sexist

attitudes on endorsement of and engagement in cyber VAWG in Study 2

Table 3.1: Loadings of cyber VAWG items on the single forced factor in Study 3

Table 3.2: Means, standard deviations, skewness, kurtosis, minimum, and maximum values of

Study 3 variables.

Table 3.3: Zero-order correlations between Study 3 variables

Table 3.4: Standardized direct and indirect effects of ideology, DPM threats, and sexist attitudes

on endorsement of and engagement in cyber VAWG in Study 3

Table 3.5: Standardized direct and indirect effects of ideology, DPM threats, and sexist attitudes

on endorsement of and engagement in cyber VAWG in Study 3 


\section{List of Figures}

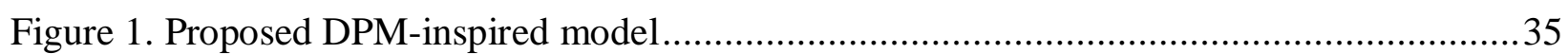

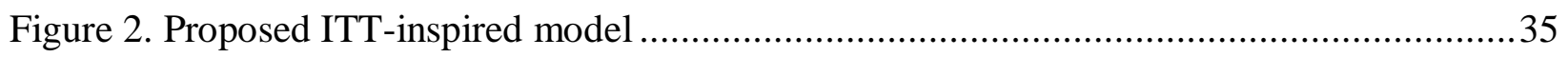

Figure 3. Scree plot produced from principal axis factoring of items assessing endorsement of

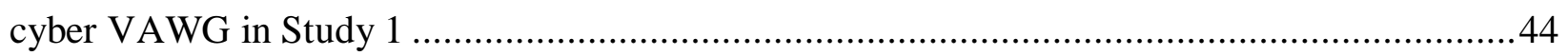

Figure 4. Scree plot produced from principal axis factoring of items assessing endorsement of

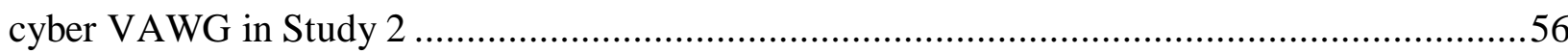

Figure 5. Scree plot produced from principal axis factoring of items assessing endorsement of cyber VAWG in Study 3 


\section{List of Appendices}

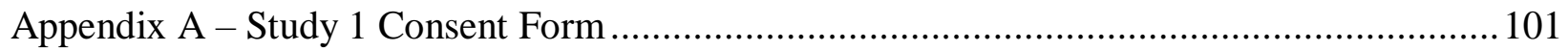

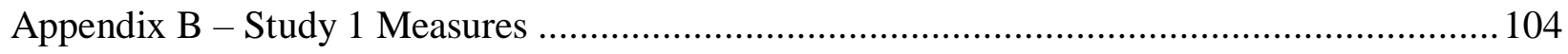

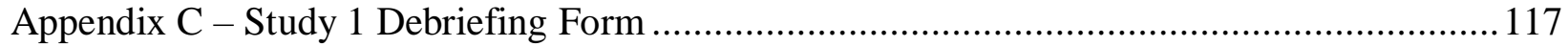

Appendix D - Factor Loadings Table for the first PAF conducted in Study 1 ..................... 120

Appendix E - Sample Online Recruitment Post...................................................... 123

Appendix F - Contact Message sent to Community Leaders .............................................. 124

Appendix $\mathrm{G}$ - Studies 2 and 3 Consent Form ...................................................... 125

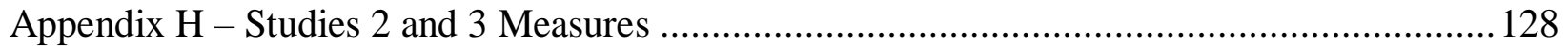

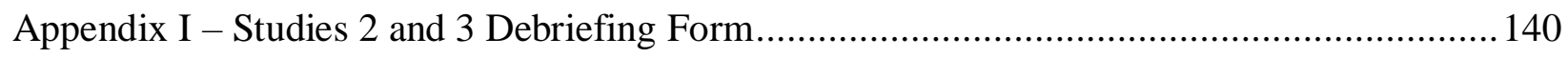

Appendix J - Studies 2 and 3 Incentive Draw Survey ................................................ 142

Appendix K - Factor Loadings Table for the first PAF conducted in Study 2 ...................... 143

Appendix L - Factor Loadings Table for the first PAF conducted in Study 3....................... 146 


\section{Introduction}

Despite the rise in Internet access and social media usage worldwide opening up innumerable opportunities for people to meet and connect with others, the same digital environments that facilitate these positive social interactions can also be exploited to carry out harmful acts of cyber-aggression. More often than not, these victims are women. The European Union's Agency for Fundamental Rights estimates that $11 \%$ of women have been subject to some form of unwanted or offensive sexually explicit e-mails, text messages, or advances on social networking sites (European Union Agency for Fundamental Rights, 2014). Further, a recent report by the United Nations, entitled "Cyber Violence Against Women and Girls," presents a call-to-action for the titular phenomenon, which has seen a rise in high profile cases in the past few years. Cyber violence against women and girls (cyber VAWG) is any electronic, gendered violence that is likely to lead to physical, sexual or psychological harm or suffering, and encompasses a wide variety of behaviours (United Nations Entity for Gender Equality and the Empowerment of Women and United Nations Development Programme, 2015).

Currently, psychological research investigating who engages in or endorses cyber VAWG is limited. The present thesis contributes to this literature by utilizing social psychological theory and research on intergroup relations to expand current understanding of cyber VAWG and create a measure for assessing engagement in and endorsement of cyber VAWG. Specifically, research on intergroup attitudes informs the notion that people behave differently towards other groups depending on how they perceive them relative to one's own group (Duckitt \& Sibley, 2009; Pratto, Sidanius, Stallworth, \& Malle, 1994; Riek, Mania, \& Gaertner, 2006; Sibley, Wilson, \& Duckitt, 2007; Stephan, 2014; Stephan \& Stephan, 2000). In particular, it has been demonstrated that higher levels of right-wing authoritarianism (RWA), or 
the belief that conventionalism, social norms, and authorities should be respected, have been demonstrated to predict more subtle, subjugating expressions of sexism (Altemeyer, 1981; Sibley et al., 2007). Similarly, higher levels of social dominance orientation (SDO), or the belief that a social hierarchy of power exists between groups of people, can predict prejudice, including sexism, particularly overt, hostile expressions of sexism (Pratto et al., 1994; Sibley et al., 2007). Various perceived threats account for these negative gender attitudes (Riek et al., 2006; Sibley et al., 2007). These threats, beliefs, and sexist attitudes can potentially catalyze acts of cyber VAWG, whereby the women and girls who are victimized represent a threatening outgroup to the perpetrators, and are subject to intergroup attitudes that result in negative outcomes. The current thesis proposes that sociopolitical ideologies, mediated by perceived threat, predict sexist attitudes, which subsequently predict endorsement of and engagement in cyber VAWG.

\section{Cyberbullying and Cyber Violence}

Cyber-aggression can take many forms, with the most familiar and well-researched form being cyberbullying. As a relatively new social phenomenon, there is little consensus among social scientists as to what constitutes cyberbullying. Cyberbullying generally refers to intentional and repeated aggressive behaviour that is communicated electronically towards an individual who cannot adequately defend themselves (Kowalski, Giumetti, Schroeder, \& Lattanner, 2014; Smith et al., 2008; Willard, 2007). This conception adheres to Olweus' (1993) classic definition of traditional bullying, whereby the one or more persons repeatedly engage in acts of aggression towards one or more others amidst an "imbalance of power" between the two parties (Cleemput \& Grigg, 2010; Olweus, 1993). Both of these conceptions characterize behaviour as aggressive, intentional, repeated, and occurring in the context of a power differential between perpetrators and victims, with the primary difference being that 
cyberbullying occurs electronically (Cleemput \& Grigg, 2010). Cyber-aggression, in comparison, more broadly refers to any intentionally aggressive behaviour that occurs electronically, with the presence of a power imbalance and repetition further specifying behaviour as cyberbullying (Cleemput \& Grigg, 2010).

The specificity and taxonomy of cyberbullying behaviours varies across psychological literature (Berne et al., 2013). A taxonomic structure created by Willard (2007) provides one of the more comprehensive attempts to define cyberbullying, and classifies behaviour into seven different types: Flaming (i.e., inciting fights online), harassment (i.e., a constant series of intentionally offending messages towards a specific target), outing and trickery (i.e., acquiring and electronically distributing personal information of another person without their consent), exclusion (i.e., refusing an individual from participating in group online activities), impersonation (i.e., pretending to be another person online and placing that person in compromising situations through the impersonator's actions), cyber-stalking (i.e., following another person electronically and repeatedly pestering them with threatening messages), and sexting (i.e., maliciously distributing private nude photos or videos of others without their consent). This structure provides a concrete and encompassing basis for the recognition of cyberbullying behaviour, and aids in its study.

Similar to how conceptions of cyberbullying overlap considerably with those of face-toface bullying, cyber VAWG has been conceptualized as a form of violence against women. The phrase "violence against women" has been used for decades prior to the United Nations formalizing the definition in 1993:

“...the term "violence against women" means any act of gender-based violence that results in, or is likely to result in, physical, sexual or psychological harm or suffering to 
women, including threats of such acts, coercion or arbitrary deprivation of liberty, whether occurring in public or in private life" (Kilpatrick, 2004; General Assembly, 1993).

The word "women" refers to women of all ages, but is commonly extended to "women and girls" for clarity. This general definition was expanded in a 2013 report on technologyrelated violence against women by The Learning Network to encompass six broad domains of behaviour that would later be designated as components of cyber VAWG: hacking, surveillance and tracking, impersonation, harassment and spamming, recruitment, and malicious distribution (Baker, Campbell, \& Baretto, 2013; United Nations Entity for Gender Equality and the Empowerment of Women and United Nations Development Programme, 2015). Hacking refers to the unauthorized or illegal access to systems and resources in an attempt to acquire, manipulate or maliciously use women's personal information (i.e., acquiring account passwords, remotely controlling computer functions, enlisting data brokers to steal information). Surveillance and tracking refers to the use of technology to stalk and track women's actions (i.e., GPS tracking via mobile phones, monitoring e-mails, recording keystrokes). Impersonation refers to adopting a woman's identity online in pursuit of acquiring private information, damaging social relationships, terrorizing the victim or creating fraudulent identity documents (i.e., impersonating someone in instant messaging applications, creating fake accounts or profiles using the identity of the victim, creating counterfeit passports). Harassment and spamming refer to repeated attempts to contact, agitate, threaten or scare women online (i.e., incessant messaging via text, e-mail, or social media). Recruitment refers to the act of luring women into compromising situations online (i.e., tricking women into human trafficking, using fake online job postings to bait women into meeting in real life). Finally, malicious distribution refers to the 
unwanted distribution of information from or about women that is intended to cause harm to them (i.e., using intimate photos or video as blackmail, making defamatory posts on social networking sites, posting propaganda supporting violence against women). Similar to Willard's (2007) taxonomy of cyberbullying behaviour, this taxonomy of cyber VAWG provides an encompassing description and classification of gendered aggression experienced by women and girls through the Internet.

It is clear that there is considerable overlap between Willard's (2007) subcategorizations of cyberbullying and The Learning Network's (2013) categorization of cyber VAWG behaviour; both share distinctions for harassment, malicious distribution of damaging material, stalking, targeted fraud, impersonation, malicious access and inflammatory interference in social relationships. The primary difference between cyberbullying and cyber VAWG, then, is that cyberbullying concerns all repeated and intentional acts of harm towards victims whereas cyber VAWG does not require acts to be repeated to fall under its umbrella. Lone acts of aggression towards a woman, such as sharing private, intimate photos of her online, can have devastating and enduring effects on her interpersonal relationships, self-esteem, sense of security, and more. In this example, because it is not a repeated behaviour, it would not classify as cyberbullying, however it is a poignant example of cyber VAWG. Furthermore, cyber VAWG specifies women and girls as the victims, informing the different grouping of behaviours within its subcategories relative to cyberbullying and recognizing the potential for cyber-aggressive behaviour to target entire populations. More specifically, whereas the targets of cyberbullying behaviour can be a group of people, acts of cyber VAWG do not necessarily have to be towards a specific group of women and can be targeted at all women. An example of this would be sharing and perpetuating misogynistic beliefs on public forums and social networking sites; doing so would not be an 
attempt to harm specific women, but an effort to discredit and threaten the welfare of women everywhere.

\section{The Role of Gender in Cyber-Aggression}

Gendered online harassment is a well-documented phenomenon in psychological literature (Barak, 2005; Barlett \& Coyne, 2014; Chisholm, 2006b; Henry \& Powell, 2015). Although there are mixed reports as to whether cyberbullying and cybervictimization occur disproportionately for different genders (for a review, see Tokunaga, 2010), a recent metaanalysis of 77 psychological studies on cyberbullying determined that gender was a small, but significant predictor of cybervictimization, with females being significantly more likely to be victimized online than males (Guo, 2016). Some studies report that nearly $60 \%$ of cyberbullying victims are female (Li, 2007). Gender differences in cyberbullying behaviour have been suggested to be moderated by age, such that females are more likely to be cyberbullies in early adolescence, but at a later point in adolescence onwards, males comprise the majority of cyberbullies (Barlett \& Coyne, 2014; Cappadocia, Craig, \& Pepler, 2013). This finding is particularly notable because traditional bullying research has historically suggested the opposite, whereby boys both bully and are bullied more than girls (Cappadocia et al., 2013; Dooley, Pyżalski, \& Cross, 2009; Forero, Mclellan, Rissel, \& Bauman, n.d.; Nansel et al., 2001).

There is also support for the notion that perceptions of harassment differ across genders and contexts, and these differences can shape people's responses (Cober et al., 2002; Fichman \& Sanfilippo, 2015). Biber and colleagues (2002) had participants rate the degree to which various behaviours represented harassment in traditional and online academic contexts. Results found that scenarios involving misogyny, the use of nicknames, and professors requesting company from their students were perceived as being more harassing when they occurred online versus in 
traditional settings. Additionally, in online contexts specifically, women were more likely than men to perceive scenarios involving sexual pictures, sexual jokes and professors requesting company of their students as being exhibitive of harassment. Similarly, Fichman and Sanfilippo (2015) presented participants with a variety of online trolling scenarios, including limited information about the troll such as their gender; participants indicated what they thought the trolls' motivations were. Notably, the authors found that men and women reacted to online trolling differently. Men were more likely to either participate in discussions with the trolls or block communications with them outright, whereas women showed a greater variety of responses, most often avoiding confrontation with or blocking the trolls in favour of ignoring them and deterring potential debates that may arise. These studies provide insight into gender differences in the perception of and reaction to cyber-aggressive behaviour, substantiating the importance of gender when examining deviant online behaviour.

Gendered harassment has also been documented in virtual online worlds. Female avatars, or $3 \mathrm{D}$ representations of users in virtual worlds, were significantly more likely to be victims of sexual harassment in comparison to their male counterparts (Behm-Morawitz \& Schipper, 2015). This finding in a context where the bully's true identity is detached from their actions bears resemblance to research in traditional bullying literature where girls are significantly more likely than boys to be victims of indirect forms of bullying, such as being the target of rumours, jokes and teasing, and significantly more likely to be the victim of these acts on a repeated basis (Carbone-Lopez, Esbensen, \& Brick, 2010). Additionally, sexual harassment, name-calling and targeting of obscene comments was increasingly likely for more sexualized female avatars, but not present at all for male ones. Gendered cyber-harassment occurring in virtual environments is a clear reflection of the uneasy reality that even when our communications are mediated through 
computers and interfaced by abstracted, partially anonymized, virtual representations of ourselves, women experience cyber-aggression differently and to a greater extent than men; further exemplifying how imperative it is that investigations of cyber VAWG are informed by these gendered differences that contrast the phenomenon from broader cyberbullying behaviour (Behm-Morawitz \& Schipper, 2015). Altogether, there is a reasonable case to be made that cyber-aggression occurs and is experienced differently when the victims are female, warranting an investigative approach that is sensitive to and focused around the unique cyberbullying experiences of female victims. Cyber VAWG adheres to these needs.

Although the effects of cyber VAWG are unmistakable, who the perpetrators are, and their psychological reasons for engaging in cyber VAWG, remain open questions. In her metaanalysis on the predictors of cyberbullying behaviour across psychological literature, Guo (2016) identified several traits that seem to be typical of cyberbullies. Typically, cyberbullies: are older males; have experience with traditional bullying behaviour; display noticeable behavioural issues; support aggression as a means to an end; frequently participate in activities online; are victims of traditional bullying; report symptoms of internalization; exhibit anti-social personality profiles; possess relatively little remorse or empathy for others; have been exposed to high parental conflict or experienced low parental supervision; are in negative school climates; have poor relationships with their peers; and are vulnerable to violent or deviant peers (Guo, 2016).

Conversely, the typical cyberbullying victim: is female; has been victimized by traditional bullying; displays high levels of depression, helplessness, stress, or loneliness; frequently participates in activities online; engages in traditional bullying; strongly internalizes psychological issues, demonstrated by resulting avoidant responses; participates in various problem behaviours; has anti-social personality disorders or psychopathic traits, low self- 
satisfaction, self-concept or self-esteem; has positive attitudes towards aggression; lives in negative family environments; shows less engagement in school; and has experienced both rejection and isolation from their peers (Guo, 2016).

Typical cyberbullies and victims of cyberbullying share many traits, including experience with traditional bullying, strong views on the value of aggression, high rates of activity online, anti-social personalities, and various symptoms of poor familial and peer interpersonal relationships (Guo, 2016). Despite this overlap, gender stands out as one of the prime distinguishers between cyberbullies and their victims, corroborating a growing body of literature that identifies males as more commonly being the cyberbullies and more involved in actual acts

of cyberbullying (Barlett \& Coyne, 2014; Calvete et al., 2010; Dehue, Bolman, \& Völlink, 2008; Dooley et al., 2009; Li, 2007). This frequent gender distinction between cyberbully and cybervictim demonstrates the need for a specialized measure of cyberbullying when females are the victims. Amidst this finding that males are more often the perpetrators and given the genderfocus of cyber VAWG in comparison to cyberbullying, it is necessary to consider the potential, more general function of sexist attitudes in this phenomenon. Importantly, both men and women can endorse sexist attitudes.

\section{Ambivalent Sexism}

Psychological conceptualizations and the measurement of sexism, or people's attitudes towards women, has evolved considerably over the past four decades (McHugh \& Frieze, 1997). Some of the most popular and well-validated measures include the Attitude Toward Women Scale (Spence \& Helmreich, 1972), The Sex Role Egalitarianism Scale (Beere, King, Beere, \& King, 1984), the Modern Sexism Scale (Swim, Aikin, Hall, \& Hunter, 1995), and the Ambivalent Sexism Inventory (Glick \& Fiske, 1996). The Attitude Toward Women Scale was 
one of the earliest attempts to determine the composition of sexist attitudes. The scale measures attitudes towards the roles of women, as well as related attributes of those gender roles, by assessing individuals' reactions to non-traditional female roles. The Sex Role Egalitarianism Scale built upon the foundation of the Attitude Toward Women Scale by including more comprehensive representations of gender roles and extending them to encompass male gender roles as well (Eagly \& Mladinic, 1989; McHugh \& Frieze, 1997). In doing so, the Sex Role Egalitarianism Scale sought to identify negative attitudes towards women, and more broadly evaluate the degree to which an individual would respond to a target person regardless of the target's sex.

Recognizing changes in the forms and expressions of sexist behaviour over the years, the Modern Sexism Scale was developed alongside the Old Fashioned Sexism Scale by Swim and colleagues (1995) to discern two conceptually different kinds of sexism. Sexism was regarded as not being uniform in its expression, and established as being either overt or covert in nature. Overt sexism, measured by the Old Fashioned Sexism Scale, represented more blatant and direct expressions of sexism that characterized historically negative attitudes towards women. These included the endorsement of traditional gender roles like previous measures of sexism, in addition to explicit beliefs that women should be treated differently from men, and are stereotypically less competent in comparison (Swim et al., 1995). However, the Modern Sexism Scale was intended to measure subtler, more covert expressions of sexism amidst growing societal and normative disapproval of overt forms of sexism, modeling itself after the Modern Racism Scale which reflected increasing societal pressure against blatant racism towards African-Americans (McConahay, 1986). People who hold modern sexist attitudes may not necessarily endorse old-fashioned sexist stereotypes and prejudices, but will instead express 
sexism through the denial of continued discrimination against women, the antagonizing women's demands and resenting women for the concessions made to compensate for institutional or societal norms of sexism (Swim et al., 1995).

Glick and Fiske (1996; 1997) proposed Ambivalent Sexism Theory after noticing a gap in existing conceptualizations of sexism. In all of the preceding conceptualizations of sexism detailed above, attitudes that are considered sexist towards women are synonymous with those that are hostile in intent. Glick and Fiske $(1996 ; 1997)$ considered sexism as being inherently ambivalent in nature because, in addition to the many forms of intentionally hostile behaviours towards women catalogued by prior interpretations of sexism, the authors proposed that behaviours that are subjectively positive in intent could also reflect sexist attitudes. These distinctions bear some similarity to those of Swim and colleagues' (1995) distinctions between overt and covert sexism in the Modern Sexism scale in that they both serve to distinguish more directly overt and explicit expressions of sexism from those that are less explicitly and outwardly antagonistic towards women.

Where they diverge, however, is that the less antagonistic forms of sexism, dubbed as being covert under Modern Sexism theory, are more often than not hostile in intent; whereas Glick and Fiske (1996) suggested that a class of sexist behaviours exist where the exhibitor believes that what they are doing is decidedly positive and wholly in the best interest of the victim. This class of behaviours and the attitudes that underlie them are perceived as being subjectively benevolent in intent, thus earning the label of benevolent sexism. Thus, whereas Swim and colleagues' (1995) sorted sexist behaviour based on how salient or concealed it was to victims, Glick and Fiske (1996)'s understanding of Ambivalent Sexism distinguishes behaviour 
based upon perpetrator's intent, and opens the concept to a new category of behaviours that ultimately lead to sexist outcomes.

As a result, Glick and Fiske (1996) created the Ambivalent Sexism Inventory, which has become one of the most widely used and comprehensive measures of sexism. It formally groups sexism into two types: hostile sexism and benevolent sexism (Glick \& Fiske, 1996, 1997). Hostile sexism encapsulates the aforementioned intentional acts of antipathy towards women that have traditionally dominated interpretations of sexism, referring specifically to attitudes that view women as incompetent and antagonistic. An example of hostile sexism would be a boss being unwilling to give a female employee a promotion to a managerial position because they do not believe that women are fit for and can be trusted with executive decision-making. Glick and Fiske (1996) noted that, generally, as a concept, hostile sexism falls in line with traditional conceptualizations of prejudice and discrimination. In comparison, benevolent sexism encompasses subjectively positive attitudes that patronize and relegate women into traditional gender roles. An example of benevolent sexism would be a co-worker complimenting a female colleague on how attractive she looks; the co-worker making the comment believes their comment is harmless, but is unaware that in making it they are potentially undermining the professionalism of the female colleague and supporting stereotypical, looks-based evaluations of women in professional settings (Glick \& Fiske, 1996). A key distinguishing aspect of benevolent sexism is that the perpetrators are not necessarily aware they are engaging in it, as the behaviours that reflect benevolent sexist attitudes are often camouflaged by prosocial behaviours (Glick \& Fiske, 1996). The subjective permissiveness of this behaviour is grounded in traditional stereotyping of women and male dominance being socialized in the perpetrators. 
Ambivalent Sexism has been demonstrated cross-culturally, lending credence to the notion that this most recent definition of sexism being the most descriptive and generalizable (Glick \& Fiske, 2001). Confirmatory Factor Analyses (CFA) of the Ambivalent Sexism Inventory (ASI) administered to 15,000 men and women, in 19 countries across 6 continents revealed that hostile sexism and benevolent sexism emerge as two distinct, but positively correlated factors (Glick et al., 2000). Furthermore, both of these factors predicted gender inequality cross-culturally and outperformed several alternative single and multi-factor (using protective paternalism, complementary gender differentiation, and heterosexual intimacy subfactors of benevolent sexism) models of sexism. The pervasiveness of this model cross-culturally is suggestive of a common societal and biological foundation for hostile and benevolent sexism across groups of people all over the world. Sexism is theorized to be rooted in the differential sexual reproductive capabilities of men and women that are a biological constant that inform gender roles globally, such as childbearing and rearing. This basis is one that may exist in online communities where geopolitical boundaries have less of an influence on social opportunities and group membership.

Both hostile and benevolent sexist beliefs relate to a variety of negative outcomes (Connor, Glick, \& Fiske, 2017), particularly rape-myth endorsement. In a study by Viki and Abrams (2002), male and female participants read vignettes describing female victims of acquaintance rape as either married or of unknown marital status. Participants higher in benevolent sexism were significantly more likely to blame the victims for the rape when the woman was identified as married. This was because victims were perceived as behaving in a deviant manner relative to how a woman should behave, reflecting support of socialized female gender norms of fidelity and purity (Abrams, Viki, Masser, \& Bohner, 2003; Viki, Abrams, Viki, 
\& Abrams, 2002). In a follow-up study, higher hostile sexism uniquely related to greater proclivity towards acquaintance rape, mediated by beliefs that the victims truly desired the sex but only declined to maintain an impression of chastity (Abrams et al., 2003).

Ambivalent sexism and cyber violence. Given the links between hostile sexism and other aggressive and violent behaviours toward women, it is reasonable to propose that hostile sexist behaviours in particular are more characteristic of the perpetrators of cyber VAWG, who often explicitly aim to subjugate and suppress female voices through aggressive displays of power and control. With cyber VAWG, there is seldom a subjective belief that the behaviours are positive and prosocial, as they are often explicitly targeted and malicious in intent. Hence, people who display higher levels of hostile sexism might be more likely to engage in or share beliefs supporting cyber VAWG, as those intentionally discriminatory attitudes towards women could serve to enable attitudes towards cyber VAWG. However, it is still possible that some of the behaviours that constitute cyber VAWG could indeed be perceived as subjectively positive, particularly those that fall under the categories of impersonation and surveillance (e.g. someone impersonating or tracking an individual without their consent believing that they are responsible for their safety). In these cases, people who display higher levels of benevolent sexism might also be more likely to engage in or share beliefs supporting cyber VAWG. It is important to note that even if a person shows higher levels of hostile sexism, it does not necessarily mean that they do not also display higher levels of benevolent sexism, as the expression of sexism is highly dependent on the social context and female victim in question. For example, a person could be highly patronizing and expectant of conformity to gender roles to their sister, but express intense antipathy towards women they do not know personally via social media outlets online. 


\section{Sociopolitical Ideology: Social Dominance Orientation and Right-Wing Authoritarianism}

Specific attitudes like hostile and benevolent sexism often originate from more general beliefs, or ideologies. Two prominent sociopolitical ideologies underlying attitudes about intergroup relations are RWA and SDO. Authoritarianism was originally conceptualized as a personality type and correspondingly measured by the Fascism, or F-Scale (Adorno, FrenkelBrunswik, Levinson, \& Sanford, 1950). After considerable scholarly review, however, the components that comprised the authoritarian personality displayed poor psychometric properties and shared more in common with a set of beliefs than dispositional traits, prompting a reconceptualization of authoritarianism as an ideology (Duckitt, 2001; Feldman \& Stenner, 1997; Goertzel, 1987; Stone, Lederer, \& Christie, 1993; Verkuyten \& Hagendoom, 1998). Altemeyer (1981) reformed the authoritarian personality as RWA - an ideological metric of authoritarianism. According to Altemeyer (1981), individuals that are higher in RWA strongly believe in obedience of, respect for, and forceful social control by authorities. Specifically, individuals that are higher in RWA display: greater conventionalism, or an attachment to social norms endorsed by society and their respective authorities; greater authoritarian submission, or a greater degree of submission to authorities that are viewed as being legitimate and endorsed by the societies they inhabit; and, greater authoritarian aggression, or aggression towards individuals or groups that authorities disapprove of.

RWA is believed to be a product of social learning, as opposed to rooting itself in improper rearing in early childhood, as theories of the authoritarian personality initially suggested (Adorno et al., 1950; Altemeyer, 1988). Altemeyer (1988, 1996) argued that this social learning begins in adolescence when cognitive development has progressed to the point where individuals can understand the sociopolitical issues that relate to the role of authorities in society 
- concepts that create the foundation for authoritarianism. However, social learning of RWA is also believed to occur in children, sourced from interactions with parents and personal experiences (Duckitt, 2001). Parents often teach their children to be obedient towards them, but also towards authorities in society. This notion of and value for obedience towards authorities consequently propagates from generation-to-generation, with each generation's parents instilling authoritarianism in their children, such that when they become parents themselves, they will engender those same beliefs in their own children. Additionally, according to Altemeyer (1981), personally witnessing interactions between people, not necessarily themselves, and authorities can shape authoritarian views. Adolescents may build positive associations between people who are obedient to authorities and positive traits or outcomes they may incur, and negative associations between people who show opposition to authorities and the negative traits or outcomes they provoke.

There is empirical support for this intergenerational transmission of authoritarianism. In a study on Belgian dyads consisting of a parent and their adolescent child, parents' higher RWA was predictive of higher RWA in their children, with each of their beliefs subsequently being predictive of modern racial prejudice (Dhont \& Van Hiel, 2012). However, the relation between authoritarian beliefs predicted by parental authoritarianism and racial prejudice was moderated by intergroup contact: adolescents with less intergroup contact showed stronger racial prejudice as a result of beliefs adopted from their parents than those with greater reported intergroup contact. Similar studies of this phenomenon have conceptually replicated this intergenerational effect of authoritarianism (Duriez \& Soenens, 2009). Additionally, studies have demonstrated that greater parental promotion of conservative goals (Duriez, Soenens, \& Vansteenkiste, 2008), need for closure (Dhont, Roets, \& Van Hiel, 2013), and engagement of social and political 
discussions with their children (Meeusen \& Dhont, 2015) contribute to the intergenerational adoption of authoritarian ideologies by children. Thus, there is considerable support of Altemeyer's notion that RWA is largely the result of social learning.

Complementing Altemeyer's work, Sidanius and Pratto (1999) proposed Social

Dominance Theory. According to Social Dominance Theory, conflicts between groups in society are minimized when those groups agree on ideologies that reinforce the superiority of one group over all others in society (Pratto et al., 1994; Jim Sidanius \& Pratto, 1999). Beliefs supporting social dominance inherently promote social inequalities, laying the foundation for subsequent discrimination, and can either be legitimized or attenuated depending on the "myths" (i.e. perceived ideologies that explain how the world works) people accept or reject. Hierarchylegitimizing myths are those that endorse and help justify social inequality between groups. These ideologies "legitimize" social inequality by normalizing the allocation of things of positive and negative social value based upon group membership. For example, under this myth, a dominant social group may be associated with the possession of wealth and gold, whereas a subordinate group may be associated with poverty and disease. Though ultimately in pursuit of reducing intergroup conflict through social reorganization, hierarchy-legitimizing myths promote discrimination and oppression, facilitating subsequent prejudice. Conversely, hierarchyattenuating myths are those that uphold social equality between groups, often by encouraging equal social standing between groups or neglecting to segregate people by group altogether. Under this myth, one may agree with statements such as "all Men are created equal," which break down divisions between people and advocate for inclusiveness and social equality. According to Social Dominance Theory, support of group-based social hierarchies emerge as a result of aggregated individual discrimination, aggregated institutional 
discrimination, and intergroup processes that facilitate discrimination (Pratto, Sidanius, \& Levin, 2006; Jim Sidanius \& Pratto, 1999). Individual acts of discrimination towards members of a different social group collectively accumulate over time, producing distinct and apparent differences in power between different social groups within a society. Similarly, the rules and actions of social institutions, such as hospitals and schools, that disproportionately and negatively affect members of a specific social group can have their effects compound over time and contribute to the development of hierarchies between differing social groups. Last, interactions between members of a subordinate social group and members of a dominant social group can contribute to the development of group-based social hierarchies. Members of subordinate groups can demonstrate behaviour asymmetry, where they engage in behaviours that contribute the subordination of their own group and supremacy of the dominant group. Examples of these behaviours include showing less in-group favouritism than those in the dominant groups do, showing out-group favouritism, demonstrating more destructive behaviour than those in dominant groups do, and possessing hierarchy-enhancing ideologies as a function of one's position within their own social group (Jim Sidanius \& Pratto, 1999). Hierarchy-legitimizing myths serve to reinforce these three processes and maintain group-based social hierarchies. SDO was developed as a way to measure endorsement of beliefs about social dominance, providing an ideological metric of how much an individual believes that intergroup relations should reflect a hierarchy of power, or inequality, between social groups (Pratto et al., 1994). Individuals who are higher in SDO believe that the ingroup they belong to is superior to and should dominate outgroups, and that this notion should be reflected in interactions between the ingroup and outgroups in the form of social inequality. SDO emerges as a result of five influential factors: status within groups, social context, stable individual differences in 
personality and temperament, gender, and socialization (Pratto et al., 2006; Sidanius \& Pratto, 1999). It is important to note that each of these factors explain effects where all other variables are assumed to be held constant, as in reality, many different factors can interact and contribute to levels of SDO. First, belonging to a dominant social group can engender greater SDO as members of the dominant group are motivated to retain the social benefits levied by the dominance of the ingroup, making them more likely to accept hierarchy-legitimizing myths that sustain that dominance. Members of dominant groups displaying higher SDO relative to subordinate groups has been demonstrated across gender, race, sexual orientation and socioeconomic status dimensions (for reviews, see: Sidanius, Cotterill, Sheehy-Skeffington, Kteily, \& Carvacho, 2017; Sidanius, Levin, Liu, \& Pratto, 2000; Sidanius \& Pratto, 1999).

Second, individual levels of SDO can vary depending on the specific group memberships that are made salient, as well as the perceived difference in social status or power between the group one identifies with and competing groups. Individuals' SDO may differ from when they are comparing their membership to a certain race (vs. other races) to when they are comparing their membership to a gender (vs. other gender) (Levin, 2004). Fundamentally different comparisons are being made when different group memberships are concerned that can inform different beliefs. Additionally, when the perceived gap in power between two social groups changes, so can the difference in SDO between members of each group. This was demonstrated in a study where among participants who perceived Protestants as having greater social status than Catholics, Protestants displayed higher SDO, and amongst participants who perceived Catholics as having greater social status than Protestants, Catholics displayed higher SDO (Levin, 2004). Third, individuals' personalities and temperaments can predict a relative stability in levels of SDO across both time and contexts. SDO has been demonstrated to show 
significantly strong $(r=.74)$ test-retest reliability in undergraduate participants across a 5-year period (Pratto et al., 2006), and strongly correlated, significant differences in mean SDO in members of various Israeli Jewish groups across primed ethnic and national contexts (Pratto et al., 1994).

Fourth, men have higher SDO relative to women as a function of societal expectations of women. Through their differing social roles in domestic labour, reproduction and child rearing, as domestic partners and relative to men, women are more likely to inhabit subordinate groups in group-based hierarchies than men, who typically assume more dominant societal roles; these differences have been observed cross-culturally (Pratto et al., 2006; Pratto \& Walker, 2004). The ultimate result of women occupying these different, more commonly subordinate societal roles in comparison to men is that men support generalized group inequalities significantly more, and likewise demonstrate higher levels of SDO relative to women (Levin, 2004; Pratto et al., 2006; Jim Sidanius et al., 2000; Jim Sidanius \& Pratto, 1999). Last, SDO is postulated to be influenced by socialization. For example, social interactions with individuals of different ethnicities could result in greater acceptance of hierarchy-attenuating myths and lower SDO, while being indoctrinated into discriminatory belief systems by others can conversely result in greater acceptance of hierarchy-legitimizing myths and increase levels of SDO (Pratto et al., 2006).

SDO is a strong predictor of generalized prejudice and more specific prejudices, such as racism or prejudice against housewives, the physically disabled, and feminists (Duckitt, 2006; Duckitt \& Sibley, 2009; Pratto, 1999; Sidanius, Bobo, Pratto, \& Bobo, 1996; Jim Sidanius \& Pratto, 1999). Furthermore, SDO is a powerful ideological predictor of intergroup attitudes towards groups that are disadvantaged, subordinate, and lower in power and status (Pratto et al., 2006); evaluations that are typical of the societal value placed on women relative to men 
throughout history (Duckitt, 2006; Duckitt \& Sibley, 2009; Sibley et al., 2007). These groups include immigrants (Esses, Dovidio, Jackson, \& Armstrong, 2001), foreigners living domestically (Wagner, van Dick, \& Zick, 2001), homosexuals (Davies, 2004; Whitley \& Lee, 2000), and women (Bates \& Heaven, 2001; Lippa \& Arad, 1999; Pratto et al., 2000; Russell \& Trigg, 2004).

Combining these robust ideological predictors of prejudice into a single model, Duckitt proposed the dual-process motivational (DPM) model of ideology and prejudice in 2001 that identified RWA and SDO as two related, but independent ideological dimensions that can together predict a variety of prejudices. Together, RWA and SDO account for a significant portion of the variance in generalized prejudice, explaining upwards of $50 \%$ of variance in some studies (Duckitt, 2001); however, little of this variance was shared, as each of the two ideologies accounted for significant, unique proportions of variance, establishing the view that they are relatively orthogonal to each other (Duckitt, 2001). Their independence was corroborated for three main reasons. First, meta-analyses of studies administering RWA and SDO measures to participants showed weak-to-non-significant overall correlations between the two (Roccato \& Ricolfi, 2005). Second, the content domains indicate that each of the scales are considerably different from each other, as RWA focuses on endorsement of within-group normative conformity and obedience to authorities and SDO focuses on beliefs towards intergroup social inequality and justifications for group dominance; reflecting distinct motivational bases for the two scales (Duckitt \& Sibley, 2009). Last, each of the two scales correlate uniquely with different social and personality variables. For example, people higher in SDO are likely to be hedonistic, value power, and view the world as being socially competitive, whereas people 
higher in RWA are uniquely likely to be religious, self-righteous, value structure and conformity, and view the world as being dangerous (Duckitt, 2001; Duckitt \& Sibley, 2009).

The DPM model specifies the relations between RWA and SDO with politics and prejudice along three hypotheses (Duckitt \& Sibley, 2009). First, according to the differential moderation hypothesis, RWA and SDO predict different reactions to social processes that impact politics and prejudice. People higher in RWA are thought to react strongly to threats to collective security. Threats to traditions and stable social structures are proposed to result in expressions of support for political parties, such as authorities, or policies that can mitigate those threats. People higher in SDO are thought to react strongly to challenges to group dominance and social inequality, similarly producing support for political parties or policies that can enforce and protect social inequalities between groups. Support for the differential moderation hypothesis has been demonstrated through differential priming of social group membership predicting different anti-immigrant attitudes for people higher in RWA and people higher in SDO. Whereas priming ingroup values and norms significantly predicted prejudice for people higher in RWA, it did not predict for those higher in SDO. Further, priming competition significantly predicted prejudice for people higher in SDO, but not those higher in RWA (Dru, 2007). Support for the differential moderation hypothesis has also been demonstrated in differential depictions of outgroups: whereas people higher in RWA hold negative attitudes toward outgroups are portrayed as being threatening to the ingroup, people higher in SDO hold negative attitudes toward outgroups portrayed as being competitive to the ingroup (Cohrs \& Asbrock, 2009).

Second, according to the differential mediation hypothesis, effects of RWA and SDO on negative outgroup attitudes should be mediated by different processes (Duckitt $\&$ Sibley, 2009). The effects of RWA should be mediated by perceived social threats such that when confronted 
with these threats from outgroups, people higher in RWA should support political institutions or policies that help lessen or protect against them. Comparatively, the effects of SDO should be mediated by the presence or threat of competitiveness or group dominance that these outgroups arouse, with people higher in SDO supporting political institutions or policies that strive to protect the social hierarchy that preserves the ingroup's dominance. Support for the differential mediation hypothesis has been found in surveying American students' reasons for supporting the Iraq War. Both those higher RWA and higher SDO predicted support for the war, but support was uniquely mediated by perceived threat of Iraq for those higher in RWA, and uniquely mediated by a competitive orientation towards Iraq for those higher in SDO (McFarland, 2005). Additionally, Duckitt (2006) showed that when outgroups were manipulated as either being threatening and socially deviant or subordinate and competitive for the dominance, participants higher in RWA and SDO, respectively, showed greater negative outgroup attitudes (Duckitt, 2006).

Last, according to the differential effect hypothesis, in addition to predicting similar perspectives on political policy and prejudice, RWA and SDO also predict distinct attitudes towards policies or outgroups (Duckitt \& Sibley, 2009). For example, with respect to politics, those higher in RWA and SDO would show greater preference for right-wing political parties; however, they would each hold exclusive reasons for their preferences. Specifically, people higher in RWA would support right-wing politics because of strong value placed on law enforcement and upholding traditional values, whereas people higher in SDO would support these same political institutions for their support of capitalist and anti-welfare policies that disproportionately favour the best interests of the dominant social class. With respect to prejudice, in addition to shared bases for negative outgroup attitudes, people higher in RWA 
would hold negative attitudes towards groups that are non-conforming and present social threats (i.e. criminals, terrorists). People higher in SDO would hold negative attitudes towards groups that are either subordinate or disadvantaged relative to the dominant group (i.e. unattractive people, obese people, mentally or physically disabled) or competing over dominance with it (i.e. protestors, feminists; Duckitt, 2006; Duckitt \& Sibley, 2007).

Of particular relevance to the present thesis, the differential effects hypothesis helps explain why RWA and SDO predict different forms of sexism, namely, benevolent and hostile sexism, respectively. As discussed above, both ideological dimensions in the DPM predict negative outgroup attitudes and prejudice in the face of different threats. Threats to ingroup norms and social cohesion motivate negative outgroup attitudes and subsequent prejudice for people higher in RWA, whereas competitive threats or threats to group dominance motivate negative outgroup attitudes and subsequent prejudice for people high in SDO. Sibley and colleagues (2007) theorized that in pursuit of the retention of tradition and maintenance of group cohesion, men who are higher in RWA should endorse benevolent sexist views that preserve traditional female gender roles and favour patriarchal social structures that have traditionally maintained order in societies. In contrast, men that are higher in SDO should endorse hostile sexist views, disparaging women in retaliation to the perceived gender competition and threats to male dominance that women pose. In a meta-analysis of six studies and test of the differential effects hypothesis on ambivalent sexism using structural equation modeling (SEM), Sibley and colleagues (2007) found moderately strong correlations between RWA and benevolent sexism and between SDO and hostile sexism. They also found that these relations held longitudinally over a period of six months. The model with predictive pathways from sociopolitical ideologies to ambivalent sexism showed a slightly better fit $(\mathrm{GFI}=.90)$ than an alternative model with 
predictive pathways from ambivalent sexism to sociopolitical ideologies $(\mathrm{GFI}=.87)$. These findings suggest that ideological beliefs may lead to sexist attitudes, and are consistent with the differential effects hypothesis of the DPM model.

Taking these findings into account, it is reasonable to expect that perpetrators of cyber VAWG hold ideological beliefs that support notions of group inequality. Specifically, gender inequality, and possessing higher SDO would contribute to individuals perceiving women as posing a competitive threat to males, subsequently serving as the mechanism behind hostile, sexist acts of cyber VAWG. Higher levels of hostile sexism and SDO predict greater sexism and sexual harassment in online video games (Fox \& Tang, 2014; Tang \& Fox, 2016). This finding is relevant to the present investigation where video gaming communities are the lenses through which I examine cyber VAWG. If higher levels of SDO elicit gendered harassment within peerto-peer or networked video games, it is possible that these behaviours exist outside of the games themselves and within the online communities that develop around them. Although unlikely, given that the acts that constitute cyber VAWG are primarily hostile in nature, it is additionally possible that people who possess higher RWA could perceive women as being socially threatening, thus eliciting benevolent sexism under the pretence that their actions are subjectively positive and in pursuit of a greater good. For example, a person may stalk or impersonate a woman via the Internet believing that their actions are in the best interests of her safety and protection, while oblivious to the notion that these actions undermine those interests, as well as her security and privacy.

To date, there has been little research on the link between cyber-aggression and prejudicial outgroup attitudes, and similarly less on cyber-aggressions where the outgroup constitutes any member of a specific gender. However, in traditional bullying research, there is 
some evidence that people who hold ideological views supporting group inequality, indexed by higher SDO, are more likely to engage in workplace bullying and discrimination (Parkins, Fishbein, \& Ritchey, 2006).

\section{Perceived Threat and Intergroup Attitudes}

As noted above, perceived threats to ingroup values and dominance are key mechanisms behind why people higher in RWA and people higher in SDO display sexist attitudes. Compared to how relevant a particular threat might be, how that threat is perceived and internalized by the individual or group to whom it is displayed is of greater psychological importance. Perceived threats can take many forms, but they all have the potential to influence intergroup attitudes and make people react negatively towards groups perceived as threatening (Riek et al., 2006).

Consistent with the differential mediation hypothesis of the DPM (Duckitt \& Sibley, 2009), there are two broad categories of perceived threats that could account for mechanisms underlying cyber VAWG: social threats and group dominance threats. As noted earlier, social threats are particularly relevant to those higher in RWA, and group dominance threats are particularly relevant to those higher in SDO (Duckitt \& Sibley, 2009). There is wide variety in the ways that these two types of threats are considered, as the particular groups, beliefs or resources being examined necessitate context-specific approaches and item development (Cohrs, 2013). In the context of the present thesis, the social threats experienced by gamers may be specific to the stability and adherence to norms of gaming communities and the broader culture

of gaming. Conversely, threats to the perceived superiority and dominance of male gamers could reflect group dominance threat.

In addition to the threats that drive negative outgroup attitudes in the DPM, Intergroup Threat Theory (Stephen \& Stephen, 2000) can also inform the different types of perceived threat 
that might predict attitudes towards cyber VAWG, as many of the threat types described within the theory reflect either social or group dominance threats, or both, as described by the DPM.

The Intergroup Threat Theory (also called Integrated Threat Theory; Stephan \& Stephan, 2000) encompasses several types of threats that fall under the umbrella of group dominance threats. In their Intergroup Threat Theory, Stephan and Stephan (2000) reconcile previous conceptions of intergroup threat together, positing that threats to ingroups can take one of three forms: perceived realistic threats, perceived symbolic threats and intergroup anxiety. Realistic threats, as described in Realistic Group Conflict Theory (Sherif \& Sherif, 1969), occur when groups compete for scarce resources and the potential for the outgroup to succeed and the ingroup to fail at securing those resources threatens the well-being of the ingroup, and results in negative attitudes towards the outgroup. Realistic threats, then, deeply reflect the competitive outlook on society that those higher in SDO hold, whereby groups are constantly competing for resources and there is a perception that the group that succeeds in acquiring them gains a competitive advantage and advances towards dominance over all other groups.

Expectedly, realistic threat correlates with higher SDO (Matthews, Levin, \& Sidanius, 2009; Morrison \& Ybarra, 2008) and mediates the relation between SDO and prejudice against Muslims (Uenal, 2016). In the context of the cyber VAWG, females who enter the traditionally male-dominated online culture of video games may represent the threatening outgroup to an ingroup comprised of a subset of male gamers who identify strongly with the traditional, male gamer image. Power and control over the traditional, male gamer identity that is at risk of becoming gender neutral (or female) could represent the resource that groups are in conflict over, prompting a realistic threat. Realistic threat accounts for a significant amount of the variance in RWA (Ruffman et al., 2016) and fully mediates the relation between RWA and prejudice 
towards international students in the United States (Charles-Toussaint \& Crowson, 2010). It is possible that the notion of outgroups succeeding where ingroups do not can threaten social cohesion of the ingroup and subsequently engender negative outgroup attitudes. Accordingly, the potential for female gamers to accrue power over male gamers, in the context of the DPM, could be perceived as a social threat to male gamers.

Symbolic threats, originally fashioned as a mechanism for explaining racism, occur when the cultural values and beliefs of an outgroup conflict with those of the ingroup, and can result in ingroup bias and negative outgroup attitudes (Sears, 1988). Similar to realistic threats, symbolic threats also describe a competitive worldview of intergroup relations, albeit instead of contending for the acquisition of resources, groups are competing over the dominance of beliefs and values. Power over dominant beliefs within a society can contribute to the power of the group that is their primary proponent. Thus, people higher in SDO should also experience greater symbolic threats from outgroups, functioning as a group dominance threat in the context of the DPM. This notion that is partially supported by the positive correlation between the two variables (Morrison \& Ybarra, 2009) and evidence that symbolic threat mediates the relation between SDO and dimensions of islamophobia (Uenal, 2016).

Further, conflicting cultural values and beliefs could also pose a threat to social conventions, cohesion, and norms, and potentially the effectiveness and collective respect for authority figures as well. In accordance with the DPM (Duckitt, 2001), it is also reasonable to expect that symbolic threat can function as a social threat in addition to being a group dominance threat. Thus, it is possible that people higher in RWA - who are sensitive to threats to traditional and stable social structures - could experience greater symbolic threat from outgroups. There is considerable evidence for this notion, as greater symbolic threat relates to greater RWA 
(Mirisola \& Russo, 2014; Ruffman et al., 2016) and mediates the relation between RWA and prejudice towards international students, gay men and lesbians when referred to as "homosexuals", and dimensions of islamophobia (Charles-Toussaint \& Crowson, 2010; Rios, 2013; Uenal, 2016). With respect to the present thesis, perceived female gamer norms, and beliefs and values that conflict with and threaten those of the male gamer status quo, could reflect a symbolic threat.

Prior to Intergroup Threat Theory, realistic and symbolic threats were thought of as competing reasons for intergroup threat (for a review, see Riek et al., 2006). Stephan and Stephan (2000), however, observed that each threat type of threat accounted for a significant, unique proportion of variance in negative outgroup attitudes. They suggested that accounting for both types of threat would provide a more comprehensive understanding of the effect of perceived threats on ingroups. In addition to reconciling two prevailing theories of threat, according to Intergroup Threat Theory, intergroup anxiety and negative stereotypes about outgroups can also uniquely contribute to variance in negative outgroup attitudes.

Intergroup anxiety comprises "feelings of uneasiness and awkwardness in the presence of outgroup members because of the uncertainty about how to behave around them" (Riek et al., 2006, p. 338; Stephan \& Stephan, 1985). This anxiety makes interactions with outgroup members threatening as they could result in socially compromising outcomes; in avoidance of these outcomes, negative outgroup attitudes are produced. This fear of being embarrassed or compromised during contact with outgroups can stem from a desire to maintain the strength of the ingroup as it is a by-product of its dominance over competing groups, sometimes fuelling avoidance or choosing not to acknowledge outgroups in an attempt to mitigate this threat. Thus, intergroup anxiety can be viewed as a group dominance threat when placed in the context of the 
DPM. Intergroup anxiety accounts for variance in SDO (Matthews et al., 2009) and mediates the relation between SDO and negative attitudes towards peers in high school with physical disabilities (Bustillos \& Silván-ferrero, 2013). The fear of repeated awkwardness or embarrassment in social interactions with outgroup members could also be interpreted as a broader threat to social cohesion and the stability of social structures. Of relevance to the present thesis, the presence of females in the gaming space may elicit intergroup anxiety; male gamers may find it uncomfortable having to accommodate female gamers within traditionally masculine gaming communities and seek to avoid this discomfort, developing and strengthening negative attitudes towards females to ensure that.

The last threat type included in Intergroup Threat Theory is the threat introduced by negative stereotypes. The presence of negative outgroup stereotypes results in the development of negative expectations of outgroup members' behaviour that can create dissonance and threatening feelings if they are not met, directly influencing negative outgroup attitudes (Eagly \& Mladinic, 1989). Negative stereotypes can function as both social threats and group dominance ones. People who are higher in RWA are more likely to value and adhere to traditional social structures that can encompass negative, traditional stereotypes; and with respect to cyber VAWG in the present investigation specifically, negative gender stereotypes. When these traditional stereotypes are threatened, people who are higher in RWA might feel a threat towards the traditional society and societal roles they are motivated to uphold, in addition to threat towards their subjective view of what makes for a cohesive, traditional society with as little deviation from norms as possible.

Conversely, this disruption of stereotypical outgroup expectations could also serve as a group dominance threat as described by the DPM. Marginalized outgroups that defy expectations 
and assume powerful or influential social roles could pose an economic, social, political or cultural threat to members of the dominant ingroup. As such, individuals that are higher in SDO and are inclined to preserve a certain hierarchy between social groups could experience a group dominance threat from a perceived incongruence between members of an outgroup and internally held negative stereotypes of them. With respect to the present research, ingroup members may stereotype females entering gaming communities, using existing terms such as "gamer girl" that hold negative connotations and subsequently create negative expectations of all females who identify as gamers. When these negative, stereotypical expectations of female gamers are not met, ingroup members may experience the aforementioned dissonance and feelings of threat towards them and fuel subsequent prejudice.

Finally, group esteem threat occurs when an outgroup threatens the ingroup's image (Branscombe \& Ellemers, 1998; Riek et al., 2006; Turner, Brown, \& Tajfel, 1979). This type of threat originates from the concept of group self-esteem, which asserts that in-group favouritism is the result of the positive effect belonging in a group has towards our individual self-esteem (Turner et al., 1979). Individuals who identify with the ingroup associate their own self-esteem with the group's image, or group esteem. Thus, in the face of a threat to the group image, members in the ingroup can choose to either no longer identify with or leave the ingroup to avoid damages to one's personal self-esteem. However, if ingroup members are not capable or motivated to do either, they can additionally choose to disparage outgroups as a defense (Branscombe \& Wann, 1994; Tajfel \& Turner, 1986). Members of the ingroup who display greater identification with the ingroup may disparage threatening outgroups to a greater degree (Branscombe, Ellemers, Spears, \& Doosje, 1999; Ouwerkerk, de Gilder, de Vries, Gilder, \& Vries, 2000; Wann, 1994). 
Group esteem threat is described similar to intergroup anxiety, in that members of a group seek to maintain a positive outward impression of their group, and attempts to undermine those impressions are viewed as threatening. However, group esteem differs from intergroup anxiety in that the resulting action to mitigate threat is not avoidance-based, and instead is characterized by either removing oneself from the group or explicitly attempting to derogate outgroups that threaten the esteem of the ingroup, and can be viewed as having relatively more group esteem when compared to the ingroup (Branscombe \& Ellemers, 1998). Hence, threats to

group self-esteem can be seen as threats to group dominance, and therefore might be experienced by individuals higher in SDO. In relation to the present investigation, a specific group of female gamers, or the mere acknowledgement that a sizeable proportion of gamers are now female, could threaten the group esteem of a subset of male gamers, motivating the denigration of female gamers. Although group esteem threat is not formally part of Intergroup Threat Theory, it was included in a recent review by Riek and colleagues (2006) as an additional predictor of negative outgroup attitudes. Because group esteem is conceptually relevant to cyber VAWG, it was included in the present thesis. All of the aforementioned types of perceived threat may serve as the justification for cyber VAWG by ingroup members, most commonly taking the form of retaliatory acts of harassment and aggression.

\section{The Present Research}

In light of recent high profile incidents of cyber VAWG in the gaming community, the current thesis used Internet gaming communities as a lens to investigate support of, and participation in, cyber VAWG. Importantly, though, this phenomenon has been documented in multiple domains and cross-culturally (United Nations Entity for Gender Equality and the Empowerment of Women and United Nations Development Programme, 2015). As the use and 
ubiquity of smartphones and social media has increased significantly over the past decade, these platforms have provided new venues for people to play video games. A by-product of this shift is an increase in video gaming among women. According to the Entertainment Software Organization, women represented a peak $48 \%$ of gamers in 2014 , with adult women comprising the largest demographic of gamers at 36\% (Entertainment Software Association, 2014). In the same year, women surpassed men as the majority gender of gamers in the United Kingdom (52\%; up from 47\% just three years ago) (Internet Advertising Bureau UK, 2014).

Coinciding with this increase is the notion that women are more likely to play games on personal computers and mobile phones; platforms that grant access to newer formats for gaming, such as browser-based games on social networking sites and smartphone apps (Grundberg \& Hansegard, 2014). Some studies suggest the proportion of female gamers is as low as $18.5 \%$ (Yee, 2017); however national surveys place the percentage as fluctuating anywhere from $38 \%$ to 52\% over the past decade (Entertainment Software Association, 2007; Internet Advertising Bureau UK, 2014). Hence, the traditional perception of gaming as a male-dominated hobby is being challenged, and necessitates an investigation into the role of gender in video gaming communities. This dramatic shift could be perceived as a threat to a subset of gamers, especially men, who feel that what was once a male domain is now experiencing an incursion from an outgroup. More specifically, members of the ingroup who perceive gaming as a male-dominated domain could view female gamers as socially threatening and of unequal social value to the ingroup.

In addition to developing a scale used to measure endorsement of cyber VAWG attitudes, three main hypotheses were proposed in the present thesis. First, it was hypothesized that higher (vs. lower) right-wing ideologies (i.e. RWA, SDO), greater perceived threats (i.e. social threat, 
group dominance threat, realistic threat, symbolic threat, intergroup anxiety, negative stereotypes, and group esteem threat), and greater ambivalent sexist attitudes (i.e. benevolent and hostile sexism), would relate to greater endorsement of and engagement in cyber VAWG (Hypothesis 1). Second, it was expected that higher (vs. lower) right-wing ideologies (i.e. RWA, SDO) would relate to greater perceived threats (i.e. social threat, group dominance threat, realistic threat, symbolic threat, intergroup anxiety, negative stereotypes, and group esteem threat) and ambivalent sexist attitudes (i.e. benevolent and hostile sexism) (Hypothesis 2).

In accordance with the DPM (Duckitt, 2001), and the differential effects and differential mediation hypotheses specifically, the present thesis tested two models evaluating threat and sexist attitudes as mediators between ideology and cyber VAWG (Hypothesis 3). One model concerned DPM threats (i.e. social threat and group dominance threat), and the other model concerned ITT-inspired threats (i.e. realistic threat, symbolic threat, intergroup anxiety, negative stereotypes, and group esteem threat). In the DPM inspired model (Figure 1), ideology (RWA and SDO) was modelled as a predictor of threat (social threat, group dominance threat), sexism (benevolent and hostile), and endorsement of and engagement in cyber VAWG. Threats were modelled as predictors of sexism, and endorsement of and engagement in cyber VAWG. Both sexist attitudes were modelled as predictors of endorsement of and engagement in cyber VAWG, and finally, endorsement of cyber VAWG was modelled as a predictor of engagement in cyber VAWG. The ITT inspired model (Figure 2) was identical to the DPM inspired model, with the exception that realistic threat, symbolic threat, intergroup anxiety, and group esteem threat replaced group dominance threat and social threat. 


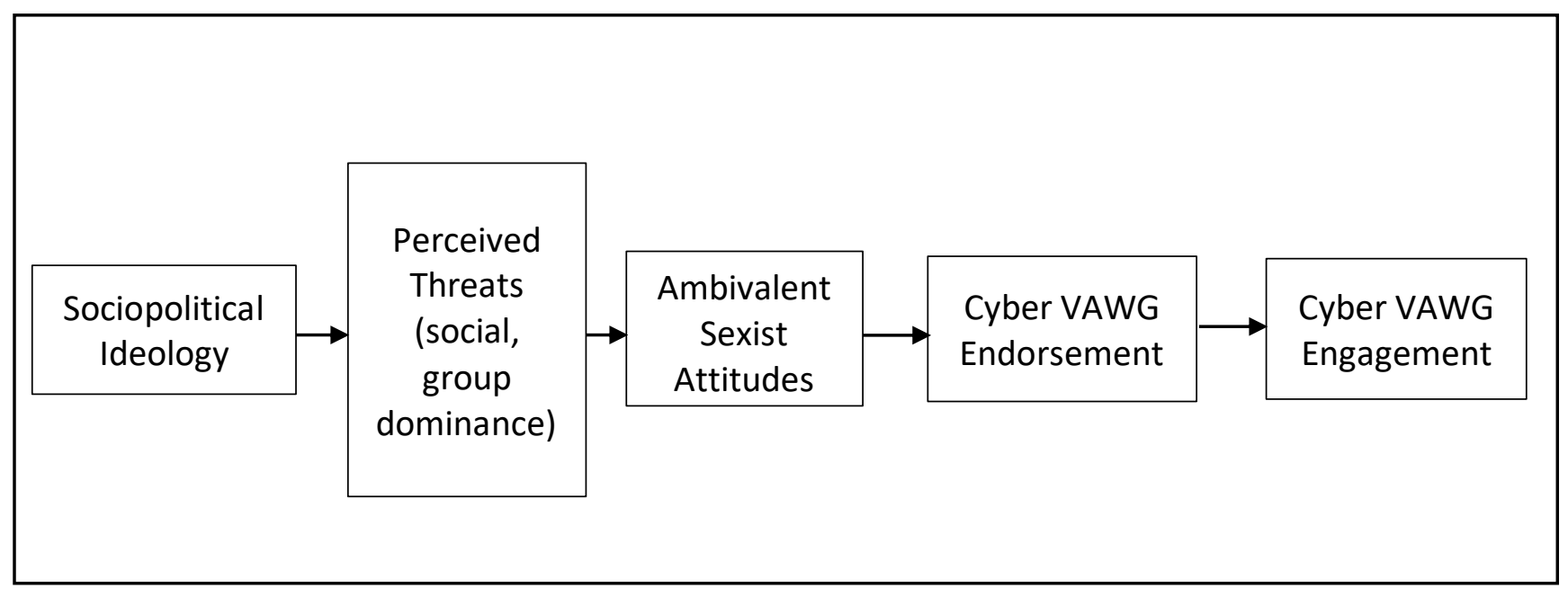

Figure 1: Proposed, DPM-inspired model of the of the impact of socio-political ideology on ambivalent sexist attitudes, mediated by perceived threats, and the impact of both ambivalent sexist attitudes and perceived threats on cyber VAWG endorsement; and subsequently, the impact of cyber VAWG endorsement on cyber VAWG engagement.

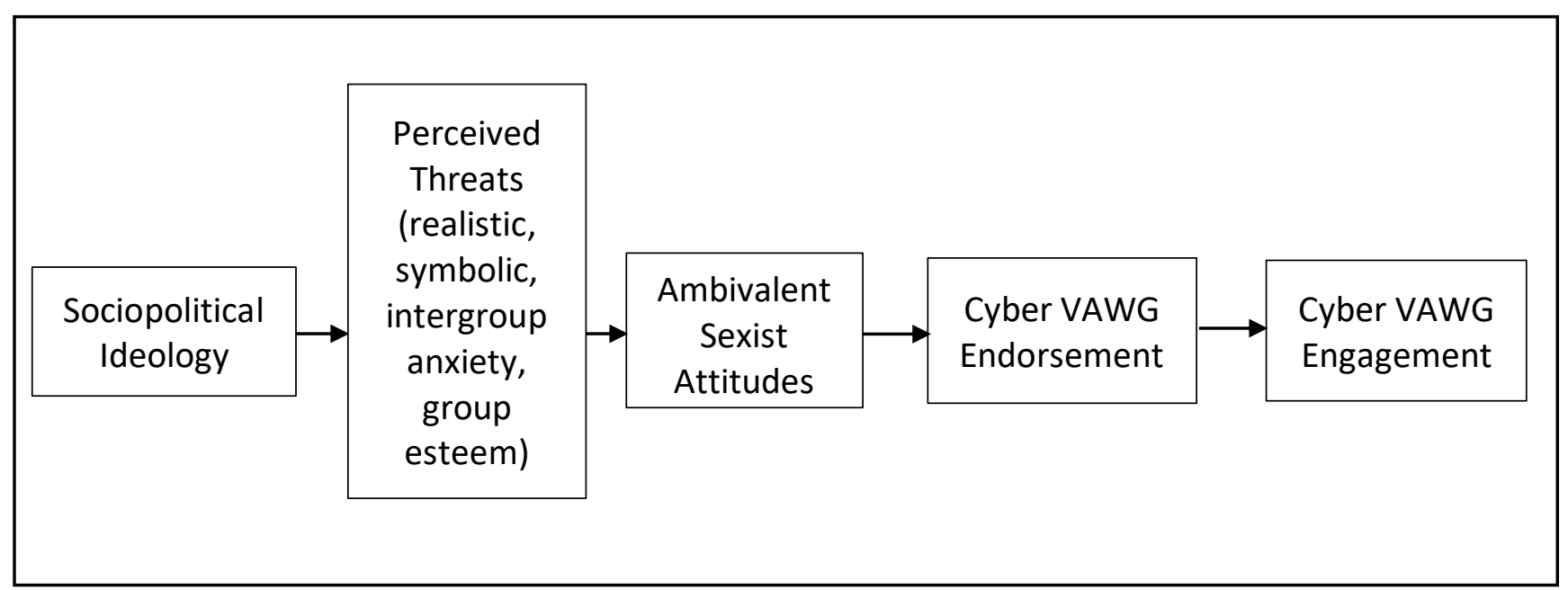

Figure 2: Proposed, ITT-inspired model of the of the impact of socio-political ideology on ambivalent sexist attitudes, mediated by perceived threats, and the impact of both ambivalent sexist attitudes and perceived threats on cyber VAWG endorsement; and subsequently, the impact of cyber VAWG endorsement on cyber VAWG engagement.

To test these hypotheses, two samples were collected, comprising three studies. In Study 1, measures of sociopolitical ideology, perceived threats, ambivalent sexist attitudes, and endorsement of and engagement in cyber VAWG were administered to a convenience sample of 
male undergraduate participants. In Study 2, the same measures were administered to a second sample, consisting of members of online video gaming communities. Part-way through data collection for Study 2, the online survey link experienced a significant increase in responding when a popular YouTuber livestreamed and uploaded a video of themselves doing the survey. Due to the probable possibility that the stream or archived video influenced responding for participants that completed the study afterwards, responses from participants in the second sample that completed the survey prior to when the video was live-streamed comprised Study 2 and responses after the start of the stream comprised Study 3. 


\section{Study 1}

Study 1 served as a preliminary test of Hypotheses 1 and 2, using a convenience sample of undergraduate participants. It was expected that right-wing ideology (RWA, SDO), perceiving female gamers as threatening, and greater sexist attitudes would relate to endorsement of and engagement in cyber VAWG (Hypothesis 1). It was also expected that right-wing ideology (RWA, SDO) would relate to perceiving female gamers as threatening and endorsement of sexist attitudes (Hypothesis 2). Hypothesis 3 was not tested in this study, as the sample size was far below the 20:1 case-to-parameter ratio required for path analyses to produce reliable estimates (Jackson, 2003). The endorsement of cyber VAWG scale, developed by myself and my supervisor, was also piloted and evaluated in Study 1. Specifically, I examined whether the factor structure of the scale would reflect a single dimension or six subscales.

\section{Method}

Participants and procedure. Participants were 46 male $^{1}$ Canadian undergraduate students that identified as gamers $\left(M_{\text {age }}=20.87, S D=.61\right.$; White/Caucasian $26.7 \%$, Filipino 13.3\%, South Asian 13.3\%, Southeast Asian 13.3\%, Chinese 8.9\%, Arab/West Asian 4.4\%, Black 2.2\%, Japanese 2.2\%, Korean 2.2\%, Other 4.4\%, 8.9\% Multiethnic) who completed a computerized survey in exchange for partial course credit. After consenting (see Appendix A), participants completed a series of questions about gaming, including: how much they identify as a "gamer," how much time they spend playing video games every week, and what their favourite game is. Participants then wrote any comments or thoughts they have about the role of women in the video gaming community. Next, participants completed dummy questions (e.g., "What colour is the sky?") as a screen for automated bots that may be completing the survey. Next,

\footnotetext{
${ }^{1}$ Female participants were also collected in this sample; however, they were excluded from analyses as the target group for cyber VAWG is women.
} 
participants completed measures of socio-political ideology, perceived threat ${ }^{2}$, sexist attitudes, endorsement of cyber VAWG, and engagement in cyber VAWG in a randomized order.

Afterwards, participants were asked whether they had heard about news stories involving female members of the video gaming community experiencing repeated harassment on social media, followed by any thoughts or feelings they may have about those news stories. Last, participants completed demographic items (see Appendix B for a copy of administered scales) and read a debriefing form (see Appendix C) explaining the specific purpose of the study.

\section{Measures.}

\section{Socio-political ideology.}

Right-wing authoritarianism. Participants completed the 22-item version of the RWA scale (Altemeyer, 2006), an updated version of Altemeyer's original scale, responding to statements (e.g., "The established authorities generally turn out to be right about things, while radicals and protestors are usually just 'loud mouths' showing off their ignorance”) on a scale from 1 (Very Strongly Disagree) to 9 (Very Strongly Agree). Scores were created by averaging the items, with higher scores indicating greater RWA $(\alpha=.82)$.

Social dominance orientation. Participants completed the 16-item version of the $\mathrm{SDO}_{7}$ scale ( Ho et al., 2015); an updated version of Pratto and colleagues' (1994) original SDO measure that distinguishes dominance (SDO-D) and egalitarianism (SDO-E) sub-dimensions of SDO. Participants indicated the degree to which they endorsed each statement (e.g., "An ideal society requires some groups to be on top and others to be on the bottom") on a scale from 1 (Strongly Oppose) to 7 (Strongly Favour). Because the present research is interested in

\footnotetext{
${ }^{2}$ Participants in all three studies completed measures of negative stereotypes, but negative stereotype threat was excluded from all analyses due to significant amounts of missing data.
} 
individuals' general SDO, all items were averaged to create an overall SDO score $(\alpha=.88)$.

Higher scores indicate greater SDO.

\section{Threat.}

Social threat. Participants completed four items modified from a social threat measure (Charlesford \& Choma, 2013; Choma, Haji, Hodson, \& Hoffarth, 2016), adapted to assess the threat posed by females in video game communities. Participants indicated the degree to which they endorsed each statement (e.g. "Female gamers undermine traditional gamer values") on a scale from 1 (Strongly Disagree) to 7 (Strongly Agree). Scores were created by averaging the three most reliable items ${ }^{3}$ (see Appendix B), with higher scores indicating greater perceived social threat $(\alpha=.87)$.

Group Dominance threat. Participants completed four items modified from a group dominance threat measure (Charlesford \& Choma, 2013; Choma et al., 2016), adapted to assess the threat posed by females in video game communities. Participants indicated the degree to which they endorsed each statement (e.g. "I hate when female gamers are portrayed as inferior to male gamers.") on a scale from 1 (Strongly Disagree) to 7 (Strongly Agree). Scores were created by averaging the three most reliable items ${ }^{4}$ (see Appendix B), with higher scores indicating greater perceived group dominance threat $(\alpha=.50)$.

Realistic threat. Participants completed three items modified from the realistic threat scale (Stephan \& Stephan, 2000; Stephan, Ybarra, \& Bachman, 1999), adapted to assess the threat posed by females in video game communities. Participants indicated the degree to which they endorsed each statement (e.g., "The rise in the number of female gamers has compromised

\footnotetext{
${ }^{3}$ Initial reliability tests showed unacceptable reliability for the scale, and suggested the removal of the fourth item (i.e. Male gamers shouldn't be threatened by female gamers).

${ }^{4}$ Initial reliability tests showed unacceptable reliability for the scale, and suggested the removal of the fourth item (i.e. The time and effort spent on protecting female gamers would be better spent on other issues).
} 
the quality and type of games that game developers make") on a scale from 1 (Strongly

Disagree) to 10 (Strongly Agree). The two most reliable items ${ }^{5}$ (see Appendix B) were averaged to create an overall index of perceived realistic threat $(r=.15)$. Higher scores indicate greater perceived realistic threat.

Symbolic threat. Participants completed five items modified from the symbolic threat scale (Stephan \& Stephan, 2000; Stephan et al., 1999), adapted to assess the threat posed by females in video game communities. Participants indicated the degree to which they endorsed each statement (e.g. "What female gamers value in their video games is problematic for longtime fans of video games") on a scale from 1 (Strongly Disagree) to 10 (Strongly Agree). An average symbolic threat score was created by averaging the four most reliable items ${ }^{6}$ (see Appendix B), with higher scores indicating greater perceived symbolic threat $(\alpha=.82)$.

Intergroup anxiety. Participants completed the intergroup anxiety scale (Stephan \& Stephan, 1985). Participants indicated how much they would experience 12 emotions when in contact with female gamers (e.g., awkward, threatened, safe, at ease) on a scale from 1 (Not At All) to 10 (Extremely). Scores were created by averaging the items, with higher scores indicating greater intergroup anxiety $(\alpha=.76)$.

Group esteem threat. Participants completed a modified version of the revised Collective Self-Esteem Scale (CSES-R; Luhtanen \& Crocker, 1992), adapted to assess the threat posed by females in video game communities. Participants indicated the degree to which they endorsed four statements (e.g., "I often regret that I belong to some of the social groups I do") on a scale

\footnotetext{
${ }^{5}$ Initial reliability tests showed unacceptable reliability for the scale, and suggested the removal of the second item (i.e. More women playing video games and having a greater say in what makes someone a gamer or not is a good thing).

${ }^{6}$ Initial reliability tests showed unacceptable reliability for the scale, and suggested the removal of the first item (i.e. The presence of female gamers can serve to help improve depictions of women in games).
} 
from 1 (Strongly Disagree) to 7 (Strongly Agree). Scores were created by averaging the three most reliable items ${ }^{7}$ (see Appendix B), with higher scores indicating greater perceived group esteem threat $(\alpha=.70)$.

Ambivalent sexism. Participants completed the 22-item Ambivalent Sexism Inventory (ASI; Glick \& Fiske, 1996), using a response scale from 0 (Disagree Strongly) to 5 (Agree Strongly). Eleven statements corresponded to endorsements of hostile sexism $(\alpha=.86$; e.g., "Women seek to gain power by getting control over men") and eleven statements corresponded to endorsements of benevolent sexism ( $\alpha=.79$; e.g., "Women should be cherished and protected by men"). Corresponding scores for each of those two subscales were averaged from relevant items. Higher scores on each of the subscales indicate greater hostile and benevolent sexism, respectively.

\section{Cyber Violence against Women and Girls.}

Endorsement of Cyber VAWG. Participants responded to 46 items measuring their endorsement of cyber VAWG, using a response scale from 1 (Strongly Disagree) to 7 (Strongly Agree). Development of the scale was conducted in consultation with my thesis supervisor, Dr. Becky Choma, and informed by existing psychological literature and reviews on cyberbullying and bullying measures (for reviews, see: Berne et al., 2013; Kowalski et al., 2014; Thomas, Connor, \& Scott, 2015; Ybarra et al., 2012). Items reflected the endorsement of attitudes related to each of the six domains of cyber VAWG behaviour: hacking, impersonation, surveillance/tracking, harassment/spamming, recruitment, and malicious distribution. Positively and negatively keyed items were included to avoid acquiescence bias. Additionally, items pertaining to attitudes towards specific female targets and women as a general target were

\footnotetext{
${ }^{7}$ Initial reliability tests showed unacceptable reliability for the scale, and suggested the removal of the second item (i.e. My willingness to identify as a gamer is diminished in the presence of female gamers).
} 
included to assess both targeted and untargeted aspects of attitudes towards cyber VAWG. 36 of these items ${ }^{8}$ were refined through exploratory factor analyses (EFA) into a parsimonious and focused scale; results are reviewed in the Results section below.

Engagement in Cyber VAWG. Participants responded to six items measuring how often they engage in common examples of behaviours that reflect each of the six domains of cyber VAWG behaviour: hacking, impersonation, surveillance/tracking, harassment/spamming, recruitment, and malicious distribution. Participants rated how frequently they engaged in each example behaviour on a scale of 1 (Never) to 7 (Very Frequently). All six items were averaged to create a score, with higher scores indicating greater reported engagement in cyber VAWG activities $(\alpha=.93)$.

\section{Results}

Cyber VAWG endorsement scale development. Principal axis factoring (PAF) was conducted on the 36 targeted items assessing endorsement of cyber VAWG, applying an oblique rotation (i.e., promax, with kappa set to 4). PAF is an approach to exploratory factor analyses where the resultant factors extracted are based upon the variance shared amongst the items (Fabrigar, Wegener, Maccallum, \& Strahan, 1999). PAF is also the most appropriate when variable distributions are non-normally distributed, as in the present sample (Costello \& Osborne, 2005; Fabrigar et al., 1999). Nine factors emerged with Eigenvalues greater than 1.00: $12.14,3.69,3.37,2.27,1.63,1.58,1.50,1.22$, and 1.14 (see Appendix D for a table of factor loadings). The Eigenvalue of the first extracted factor was considerably larger than the subsequent factors, and examination of the scree plot, shown in Figure 3, suggested extraction of

\footnotetext{
${ }^{8}$ The 36 items representing targeted cyber VAWG attitudes were included in EFAs for all studies. The remaining 10 items reflected untargeted cyber VAWG attitudes, which is conceptually distinct from targeted cyber VAWG attitudes, and were too similar to items from other included scales, and thus were excluded from analyses in all three studies.
} 
a single factor (Factor 1) representing support of cyber VAWG that accounts for $33.72 \%$ of the variance.

As the items loaded predominantly onto one factor, another PAF forcing the extraction of one factor was conducted. The loadings for each item on the single forced extracted factor are shown in Table 1.1. A cut off of .32 for factor loadings was used, as weaker loadings in factor analyses can contribute to the extraction of unstable factors (Costello \& Osborne, 2005). Of the 36 items, 28 loaded stronger than .32 onto the single extracted factor, and the 8 items that did not load stronger than .32 were dropped. Thus, the 28 items with loadings stronger than .32 were selected and averaged in the computation of the endorsement of cyber VAWG scale $(\alpha=.92)$. Higher scores on the scale indicated greater endorsement of cyber VAWG. 


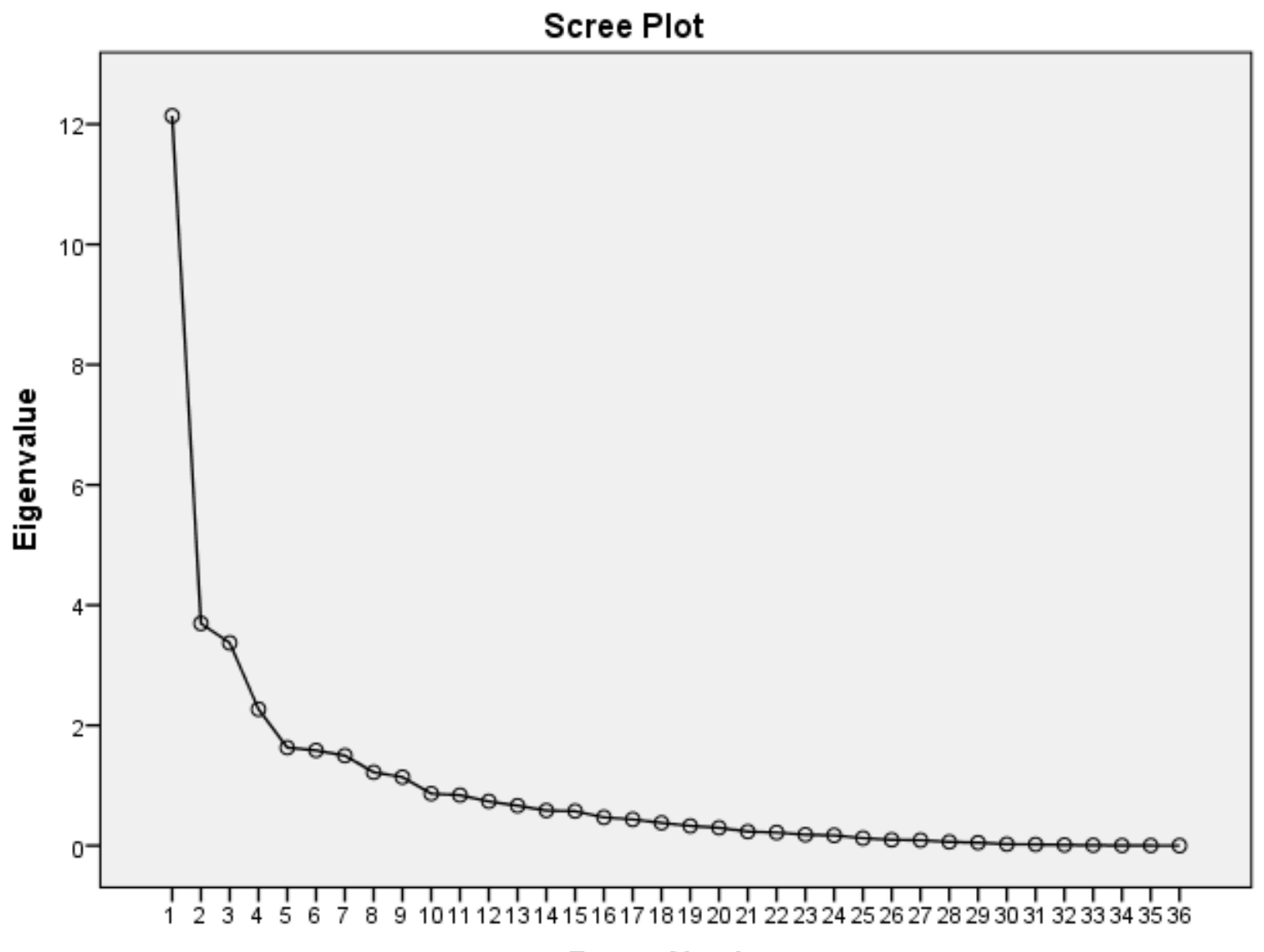

Factor Number

Figure 3. Scree plot produced from principal axis factoring of items assessing endorsement of cyber VAWG in Study 1. 
Table 1.1

Loadings of cyber VAWG items on the single forced factor in Study 1

\begin{tabular}{|c|c|}
\hline Item & Loading \\
\hline There is nothing wrong with using fake advertisements or profiles to trick a woman online. (RC) & .901 \\
\hline $\begin{array}{l}\text { It's okay to create new, anonymous online profiles or accounts for the sole purpose of using them to spam a woman with } \\
\text { messages. (HS) }\end{array}$ & .874 \\
\hline It is okay to share intimate photos or videos of a woman online without her consent. (MD) & .869 \\
\hline It is okay to trick a woman into a compromising situation using the Internet. (RC) & .853 \\
\hline There's nothing wrong with making unprovoked sexual remarks towards or sexual requests of a woman on the Internet. (HS) & .846 \\
\hline If I have figured out the password to a woman's account or profile, I can do what I want with it. (IM) & .845 \\
\hline There's nothing wrong with threatening real life violence to a woman on the Internet. (HS) & .821 \\
\hline It is okay to mislead a woman online. (RC) & .813 \\
\hline It is okay to impersonate someone online to learn more about a woman. (IM) & .771 \\
\hline $\begin{array}{l}\text { Threatening to release private information about a woman onto the Internet in order to get my way is acceptable thing to do. } \\
\text { (MD) }\end{array}$ & .743 \\
\hline To get information about a woman, it's okay to pose as her online. (IM) & .741 \\
\hline There is no problem with telling a woman to kill herself on the Internet. (HS) & .711 \\
\hline Obtaining a woman's personal information online without her permission is never okay. (HC) & .663 \\
\hline It is acceptable to gain unauthorized access to a woman's information on the Internet. (HC) & .604 \\
\hline On the Internet, to convince a woman of something, sometimes you have to say things that are not entirely true. (RC) & .572 \\
\hline If I want to access information about a woman online, I can do what I need to do to acquire it. (HC) & .537 \\
\hline Continually monitoring the activities of a woman online is totally fine. (ST) & .532 \\
\hline I'm at liberty to send intimate photos of myself to any woman I choose. (MD) & .468 \\
\hline Even if I can, I am not free to bypass security to get information about a woman online. (HC) & .460 \\
\hline I do not have the right to spread fake stories or rumors about a woman online. (MD) & .448 \\
\hline It is fine to use the Internet to contact a woman even though she does not want to be contacted. (HS) & .424 \\
\hline There is potential harm in repeatedly messaging, calling or texting a woman. (HS) & .401 \\
\hline It is okay to gain access to a live stream of a woman's webcam without her knowledge. (HC) & .394 \\
\hline It is okay to use the Internet to tell a woman what I think about her, regardless of if she wants to know or not. (HS) & .377 \\
\hline It is unacceptable to use the Internet to trace where a woman has been. (ST) & .355 \\
\hline Spamming a woman's e-mail, social media, phone or voicemail with messages is wrong. (HS) & .345 \\
\hline It is not okay to use the Internet to track down a woman. (ST) & .335 \\
\hline I have the right to express my anger towards a woman online as frequently as I wish. (HS) & .320 \\
\hline Twisting the truth about a woman online for my personal purposes is not right. (MD) & .311 \\
\hline There is nothing wrong with using the Internet to trace a woman's history. (ST) & .271 \\
\hline It is not appropriate to forge identifying information of a woman on the Internet to learn more about her. (IM) & .132 \\
\hline It is not okay to use GPS or location tracking to find out where a woman is right now. (ST) & .119 \\
\hline It is unacceptable to bend the truth on the Internet to get the information you want out of a woman. (RC) & .098 \\
\hline Pretending to be someone else when contacting a woman on the Internet is not okay. (IM) & .084 \\
\hline Just because someone is telling a woman what they think, it does not mean it is threatening or intimidating. (HS) & -.057 \\
\hline If a woman has an online presence, it is okay for me to use information she puts out there to learn more about & -.029 \\
\hline
\end{tabular}

Note. $N=46$. Loadings are derived from forced extraction onto a single factor. $(\mathrm{HC})=$ Hacking; $(\mathrm{IM})=$ Impersonation; $(\mathrm{ST})=$ Surveillance $\&$ Tracking; $(\mathrm{HS})=$ Harassment $\&$ Spamming; $(\mathrm{RC})$ $=$ Recruitment $(\mathrm{MD})=$ Malicious Distribution . 
Correlations with ideology, threat, sexism, and engagement. Means, standard deviations, skewness, and kurtosis statistics for Study 1 variables are shown in Table 1.2, and zero-order correlations among Study 1 variables are displayed in Table 1.3. Upon examining skewness values and consulting variable histograms, social threat, group dominance threat, and endorsement of and engagement in cyber VAWG showed negative skews (i.e. scores were generally low, showing lower perceived threat, endorsement and engagement in cyber VAWG); therefore, bootstrapping was performed using 1000 samples. Consistent with Hypothesis 1, higher RWA, SDO, realistic threat, symbolic threat, intergroup anxiety, social threat, and hostile sexism related to greater endorsement of cyber VAWG. Inconsistent with Hypothesis 1, greater group esteem threat, group dominance threat, and benevolent sexism did not relate to greater endorsement of cyber VAWG. Only higher SDO and greater symbolic threat related to greater engagement in cyber VAWG, providing partial support for Hypothesis 1. Higher RWA related to greater realistic threat, symbolic threat, group esteem threat, group dominance threat, and benevolent sexism, consistent with Hypothesis 2. Contrary to Hypothesis 2, higher RWA did not relate to greater intergroup anxiety and social threat, and greater RWA additionally related to greater hostile sexism. Higher SDO related to greater realistic threat, symbolic threat, intergroup anxiety, and hostile sexism, but not group esteem threat, social threat, group dominance threat, or benevolent sexism. Last, greater endorsement of cyber VAWG was also related to greater engagement in cyber VAWG. 
Table 1.2

Means, standard deviations, skewness, kurtosis, minimum, and maximum ${ }^{9}$ values of Study 1 variables

\begin{tabular}{lcccccccccccc}
\hline & RWA & SDO & REAL & SYMB & ANX & EST & SOC & DOM & BS & HS & END & ENG \\
\hline M & 2.91 & 2.69 & 3.55 & 3.76 & 2.86 & 2.09 & 1.50 & 2.42 & 3.23 & 2.98 & 1.75 & 1.33 \\
SD & .99 & .94 & 1.81 & 1.89 & .96 & 1.11 & .92 & 1.12 & .87 & .91 & .77 & .82 \\
Min & 1.17 & 1.00 & 1.00 & 1.00 & 1.40 & 1.00 & 1.00 & 1.00 & 1.09 & 1.09 & 1.00 & 1.00 \\
Max & 6.42 & 4.47 & 7.00 & 8.75 & 5.70 & 4.67 & 4.67 & 7.00 & 4.91 & 4.82 & 4.32 & 4.33 \\
Skewness & .80 & -.10 & -.06 & .28 & .69 & .70 & 2.18 & 1.41 & -.12 & .02 & 1.77 & 2.89 \\
Kurtosis & 1.98 & -.75 & -1.10 & -.52 & .42 & -.67 & 4.38 & -.67 & -.40 & -.43 & 2.86 & 7.44 \\
\hline
\end{tabular}

Note. $N=46$. Bootstrapping with 1000 samples was used with $95 \%$ confidence intervals. $* *$ indicates the correlation is significant at $p<.01, *$ indicates the correlations is significant at $p<$ .05. RWA = right-wing authoritarianism; $\mathrm{SDO}=$ social dominance orientation; REAL = realistic threat; SYMB = symbolic threat; ANX = intergroup anxiety; EST = group esteem threat; SOC = social threat; DOM = group dominance threat; $\mathrm{BS}=$ benevolent sexism; HS = hostile sexism; $\mathrm{END}=$ endorsement of cyber VAWG; ENG = engagement in cyber VAWG.

\footnotetext{
${ }^{9}$ Scores for all scales could theoretically range from 1 to 7 , with the exception for realistic threat and symbolic threat which could range from 1 to 10, and engagement in cyber VAWG, and benevolent and hostile sexism which could range from 1 to 6 .
} 
Table 1.3

Zero-order correlations between Study 1 variables

\begin{tabular}{|c|c|c|c|c|c|c|c|c|c|c|c|}
\hline Variable & 1 & 2 & 3 & 4 & 5 & 6 & 7 & 8 & 9 & 10 & 11 \\
\hline 1. RWA & - & & & & & & & & & & \\
\hline 2. SDO & .16 & - & & & & & & & & & \\
\hline 3. REAL & $.51 * *$ & $.37 *$ & - & & & & & & & & \\
\hline 4. SYMB & $.43 * *$ & $.38 * *$ & $.64 * *$ & - & & & & & & & \\
\hline 5. ANX & .19 & $.42 * *$ & $.46^{* * *}$ & $.38 * *$ & - & & & & & & \\
\hline 6. EST & $.35^{*}$ & .09 & $.43 *$ & $.33^{*}$ & .09 & - & & & & & \\
\hline 7. SOC & .13 & .28 & .18 & .18 & .26 & $.53 * *$ & - & & & & \\
\hline 8. DOM & $.50 * *$ & $<.01$ & .24 & .08 & -.05 & $.35^{*}$ & .28 & - & & & \\
\hline 9. BS & $.49 * *$ & .06 & $.54 * *$ & .44 & .24 & $.56^{* *}$ & .25 & .20 & - & & \\
\hline 10. HS & $.55 * *$ & $.39 * *$ & $.61 * *$ & $.34 *$ & $.43^{* *}$ & $.30^{*}$ & .18 & .19 & $.39 * *$ & - & \\
\hline 11. END & $.35 *$ & $.45^{* *}$ & $.32 *$ & $.29 *$ & $.41 * *$ & .20 & $.57 * *$ & .27 & .06 & $.31 *$ & - \\
\hline 12. ENG & .25 & $.45^{* *}$ & .26 & $.38 * *$ & .19 & -.01 & .18 & .07 & .13 & .13 & $.61^{* *}$ \\
\hline
\end{tabular}

Note. $N=46$. Bootstrapping with 1000 samples was used with $95 \%$ confidence intervals. $* *$ indicates the correlation is significant at $p<.01$, * indicates the correlations is significant at $p<$ .05. RWA = right-wing authoritarianism; $\mathrm{SDO}=$ social dominance orientation; $\mathrm{REAL}=$ realistic threat; SYMB = symbolic threat; ANX = intergroup anxiety; EST = group esteem threat; $\mathrm{SOC}=$ social threat; DOM = group dominance threat; $\mathrm{BS}=$ benevolent sexism; HS = hostile sexism; $\mathrm{END}=$ endorsement of cyber VAWG; $\mathrm{ENG}=$ engagement in cyber VAWG. 


\section{Discussion}

The goal of Study 1 was to provide a preliminary test of Hypotheses 1 and 2, and evaluate the cyber VAWG scale. Factor analyses suggested a single dimensional construct for endorsement of cyber VAWG, despite items reflecting six different domains of behaviour, based on The Learning Network and United Nations' classifications (Baker et al., 2013; United Nations Entity for Gender Equality and the Empowerment of Women and United Nations Development Programme, 2015). The small sample size of men limited the extent to which the scale could be refined. It is possible that the homogeneity of the sample, consisting entirely of undergraduate males, also influenced the factor loadings. Because of this possibility, all of the items addressing endorsement of cyber VAWG administered in Study 1 were retained in Study 2, rather than dropping the items with loadings lower than .32 .

Consistent with Hypothesis 1, right-wing ideology and perceived threat (with the exception of group esteem threat and group dominance threat) related to greater endorsement of cyber VAWG. Therefore, consistent with previous research showing that RWA and SDO relate to harmful attitudes towards women (Sibley et al., 2007), Study 1 provides preliminary evidence that this relation extends to gendered, aggressive online behaviours. Further, fears that female gamers may be gaining power over the gamer identity, may be awkward to interact with, and compromise traditional gamer values and social structures may additionally play a role in endorsing cyber VAWG behaviours. Hostile, but not benevolent sexism, also related to endorsement of cyber VAWG. It is not surprising that hostile sexism would relate more strongly to cyber VAWG given that hostile sexist attitudes are subjectively negative, such that they are derogatory in intent and effect (Glick \& Fiske, 1996). Behaviours that encompass cyber VAWG are overwhelmingly either malicious in intent (e.g. online harassment) or the negative 
consequences that result from the behaviours are clear (e.g. compromising a woman's security by hacking her profile). Benevolent sexism, on the other hand, is subjectively positive, meaning that people who espouse related attitudes and engage in benevolent behaviours do so with positive intentions, often with the understanding that there are no negative consequences of those behaviours. Finally, with respect to engaging in cyber VAWG, SDO, symbolic threat, and endorsement of cyber VAWG emerged as the most relevant correlates.

Partially consistent with Hypothesis 2, RWA and SDO related to perceiving female gamers as posing a realistic and symbolic threat, as expected, and consistent with literature on RWA (Charles-Toussaint \& Crowson, 2010; Ruffman et al., 2016) and SDO (Crowson, 2009; Uenal, 2016). RWA also related to group dominance threat, inconsistent with the DPM (Duckitt, 2001). Concerning relations with sexist attitudes, in line with previous research, RWA related to higher benevolent sexism and SDO related to higher hostile sexism (Sibley et al., 2007). RWA, however, also related to hostile sexism, though research typically finds that SDO relates particularly and more strongly to hostile sexism (Sibley et al., 2007). SDO, but not RWA, was also linked with greater anxiety about interacting with female gamers conceptually replicating other work (Bustillos \& Silván-ferrero, 2013; Matthews et al., 2009). Inconsistent with predictions, SDO did not relate to group dominance threat and group esteem threat.

In summary, there was evidence for links between ideology and cyber VAWG, as expected; however, there were several non-significant and unexpected relations. These findings must be interpreted with caution as power is low and the sample homogenous. Nonetheless, Study 1 provides preliminary evidence that endorsement of cyber VAWG can be summarized by a single construct representing support of cyber VAWG, and that endorsement of and engagement in cyber VAWG are associated with right-wing ideologies, perceptions of female 
gamers as threatening, and hostile sexist attitudes. As noted above, one key limitation of Study 1 is the sample: it was a small unrepresentative sample of undergraduate men. Study 2 sought to address this limitation. 


\section{Study 2}

The purpose of Study 2 was to examine the properties of the cyber CAWG endorsement scale and test Hypotheses 1,2 and 3 in a sample more representative of the gaming community, in particular, a community sample of members of online video gaming communities. As noted earlier, unexpectedly, approximately 26 hours after recruitment for Study 2 began and the online survey link was initially posted to all five online gaming communities, the survey link went 'viral': A popular YouTuber streamed themselves doing the survey to thousands of subscribers. The stream was one hour and 35 minutes in length, and was archived to YouTube at 9:23PM when the stream concluded. A significant increase in the response rate for the survey was detected shortly after the time of archival. The length of the stream was deducted from the archival time to deduce the start time of the stream, at approximately 7:48 PM. This time (7:48pm) was used as a conservative boundary for participants whose responses may have been influenced by the stream. In total, 14,396 responses were collected. This sample was split into two studies: Study 2 is comprised of participants who completed the survey prior to the time the stream began (i.e. before 7:48pm), and Study 3 is comprised of participants that completed the survey after the stream began (i.e. after 7:48pm).

\section{Method}

Participants and procedure. Participants were recruited through online video gaming communities. Links to the survey were posted simultaneously to five popular gaming boards and forums (see Appendix E for example post) with prior consent from community leaders (see Appendix F for the request sent to community leaders). Snowball method techniques were employed in recruiting (i.e. the posts encouraged participants to share the survey link with other gamers after completing it themselves). 
A total of 815 responses were recorded for the survey before the survey went viral.

Participants were removed from the original sample for: not consenting to participate (153), incorrectly answering "dummy” questions designed to screen for automated responding (163), withdrawing part-way through the study (181), opting out of having their responses included (5), taking less than 10 minutes or longer than 3 hours to complete ${ }^{10}(20)$, having duplicate responses from the same computer (3), demonstrating acquiescent responding on more than one scale ${ }^{11}(4)$, and missing a significant number of responses for items (10). The final sample for Study 2 comprised 276 participants $\left(M_{\text {age }}=27.64, S D=6.73\right.$; Gender - Male: $87.3 \%$, Female: $8.4 \%$, Other: 4.4\%; Nationality - United States: 47.5\%, Canada 10.7\%, United Kingdom 10.0\%, Germany 5.4\%, Brazil 1.5\%, France 1.1\%, Mexico 1.1\%, Japan 0.8\%, South Korea 0.4\%, Spain 0.4\%, Other 21.1\%; Ethnicity - White/Caucasian 61.4\%, Latin American 7.7\%, South Asian 3.3\%, Black 2.6\%, Chinese 2.6\%, Filipino 1.5\%, Southeast Asian 1.5\%, Arab/West Asian 0.7\%, Japanese $0.4 \%$, Korean $0.4 \%$, Other $7.0 \%, 11.0 \%$ Multiethnic).

Measures. After consenting to participate (see Appendix G), participants completed the same set of measures administered in Study 1; however, a subset of items was used for each measure to accommodate time constraints for online community participants. The items that demonstrated the greatest reliability and face validity in Study 1 were selected for inclusion in

\footnotetext{
${ }^{10}$ The lower inclusion boundary for time spent completing the survey was determined from pilot testing the survey to establish the minimum amount of time it would take someone to read through and respond to the survey in its entirety. Due to participants writing considerably lengthy responses on the open-ended questions, the upper inclusion boundary for time spent completing the survey was determined by looking at quartiles of completion time. The upper inclusion boundary represents a detected jump in completion time and is designed to distinguish participants who may have spent a lot of time writing responses from those who may have closed the survey link and returned to it later.

${ }^{11}$ Acquiescent responding was determined by creating a difference score between the mean for negatively keyed items (after reversing them) on measures that used a 7-point agree-disagree Likert-type scales, and the mean for positively keyed items on those same measures. Participants with difference scores higher than 4 (i.e. where a response of 2, or "disagree," would be acquiesced into a response of 6, or "agree," and vice versa) on more than one scale were removed.
} 
Study 2 (see Appendix H for a copy of the scales). Participants completed: a 12-item version of the RWA scale ( $\alpha=.90$; Altemeyer, 1981, 1988, 1996, 2006), an 8-item version of the $\mathrm{SDO}_{7}$ scale ( $\alpha=.87$; Ho et al., 2015), a 2-item realistic threat scale $(r=.53$; Stephan $\&$ Stephan, 2000; Stephan, Ybarra, \& Bachman, 1999), 2-items from the symbolic threat scale $(r=.69$; Stephan \& Stephan, 2000; Stephan et al., 1999), a 6-item intergroup anxiety scale $(\alpha=.77$; Stephan \& Stephan, 1985), a 2 -item group esteem threat scale $(r=.49$; CSES-R; Luhtanen \& Crocker, 1992), a 2-item social threat scale ( $r=.62$; Charlesford \& Choma, 2013; Choma et al., 2016), a 2-item group dominance threat scale ( $r=.75$; Charlesford \& Choma, 2013; Choma et al., 2016), a 12-item version of the ASI (benevolent sexism $\alpha=.83$; hostile sexism $\alpha=.92$; Glick \& Fiske, 1996; Rollero, Glick, \& Tartaglia, 2014), the 46-item measure of endorsement of cyber VAWG, and the 6-item measure of engagement in cyber VAWG $(\alpha=.82)$. Three items assessing group esteem threat were administered, however only the two most reliable were included in the computation of the group esteem threat score because inclusion of the third item resulted in unacceptable reliability (see Appendix H). Last, participants read a debriefing form (see Appendix I) explaining the specific purpose of the study and were invited to click a separate link to enter their details to be entered into a draw to win one of five gift cards valued at \$25 CAD to their preferred digital video gaming store (see Appendix J).

\section{Results}

Cyber VAWG endorsement scale development. Similar to Study 1, PAF was conducted on the 36 targeted items assessing endorsement of cyber VAWG, applying an oblique rotation (i.e., promax, with kappa set to 4). Whereas 7 factors showed Eigenvalues greater than 1 (12.04, 2.54, 1.76, 1.70, 1.42, 1.18, 1.02; see Appendix K for a table of factor loadings), examination of the scree plot, shown in Figure 4, revealed a single, predominant factor (Factor 1) 
representing attitudes towards cyber VAWG with an Eigenvalue of 12.04 that accounted for $33.44 \%$ of the variance. As the items loaded primarily onto one factor, another EFA forcing the extraction of one factor was conducted. The loadings for each item on the single forced extracted factor are shown in Table 2.1. All 36 items loaded stronger than .32 (see Costello \& Osborne, 2005). Therefore, the 36 items that comprised the extracted factor were averaged to produce a single cyber VAWG endorsement scale $(\alpha=.93)$. Higher scores on the scale indicated greater endorsement of cyber VAWG. 


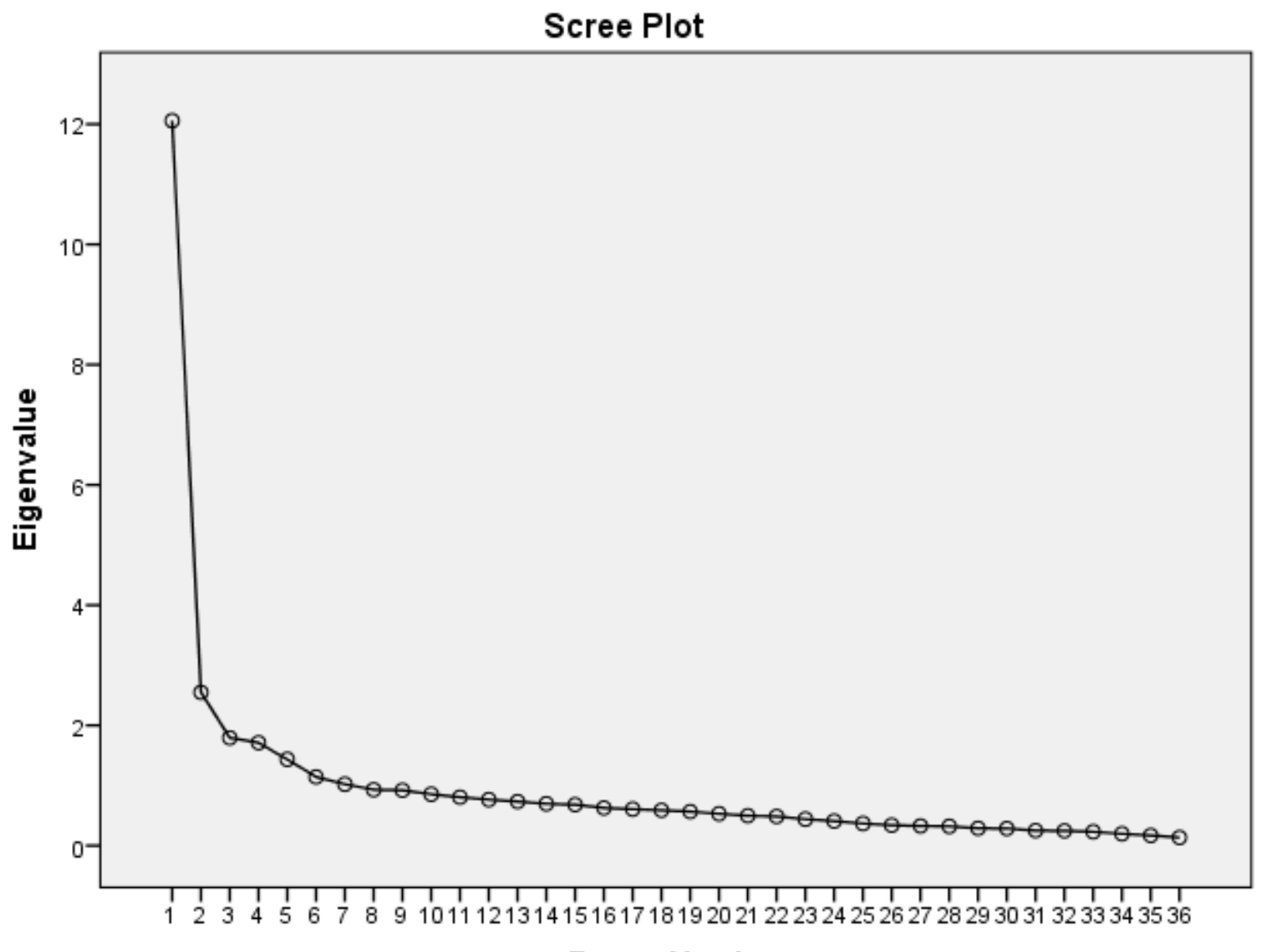

Factor Number

Figure 4. Scree plot produced from principal axis factoring of items assessing endorsement of cyber VAWG in Study 2. 
Table 2.1

Loadings of Cyber VAWG items on the single extracted factor in Study 2

\begin{tabular}{|c|c|}
\hline Item & Loading \\
\hline It is okay to trick a woman into a compromising situation using the Internet. (RC) & .795 \\
\hline $\begin{array}{l}\text { It's okay to create new, anonymous online profiles or accounts for the sole purpose of using them to spam a woman with messages. } \\
\text { (HS) }\end{array}$ & .750 \\
\hline There is nothing wrong with using fake advertisements or profiles to trick a woman online. (RC) & .750 \\
\hline There's nothing wrong with making unprovoked sexual remarks towards or sexual requests of a woman on the Internet. (HS) & .736 \\
\hline It is fine to use the Internet to contact a woman even though she does not want to be contacted. (HS) & .693 \\
\hline It is okay to mislead a woman online. (RC) & 692 \\
\hline To get information about a woman, it's okay to pose as her online. (IM) & .648 \\
\hline It is okay to use the Internet to tell a woman what I think about her, regardless of if she wants to know or not. (HS) & 648 \\
\hline There is no problem with telling a woman to kill herself on the Internet. (HS) & .642 \\
\hline Continually monitoring the activities of a woman online is totally fine. (ST) & .640 \\
\hline If I have figured out the password to a woman's account or profile, I can do what I want with it. (IM) & .635 \\
\hline There is potential harm in repeatedly messaging, calling or texting a woman. (HS) & 632 \\
\hline I do not have the right to spread fake stories or rumors about a woman online. (MD) & .631 \\
\hline It is okay to share intimate photos or videos of a woman online without her consent. (MD) & .623 \\
\hline On the Internet, to convince a woman of something, sometimes you have to say things that are not entirely true. (RC) & .602 \\
\hline I have the right to express my anger towards a woman online as frequently as I wish. (HS) & .594 \\
\hline I'm at liberty to send intimate photos of myself to any woman I choose. (MD) & .579 \\
\hline It is okay to impersonate someone online to learn more about a woman. (IM) & .579 \\
\hline Obtaining a woman's personal information online without her permission is never okay. (HC) & .572 \\
\hline There is nothing wrong with using the Internet to trace a woman's history. (ST) & .560 \\
\hline Spamming a woman's e-mail, social media, phone or voicemail with messages is wrong. (HS) & .550 \\
\hline There's nothing wrong with threatening real life violence to a woman on the Internet. (HS) & .486 \\
\hline If a woman has an online presence, it is okay for me to use information she puts out there to learn more about her. (ST) & .480 \\
\hline It is not okay to use the Internet to track down a woman. (ST) & .465 \\
\hline If I want to access information about a woman online, I can do what I need to do to acquire it. (HC) & .455 \\
\hline It is not okay to use GPS or location tracking to find out where a woman is right now. (ST) & .429 \\
\hline It is okay to gain access to a live stream of a woman's webcam without her knowledge. (HC) & .427 \\
\hline Twisting the truth about a woman online for my personal purposes is not right. (MD) & .427 \\
\hline Threatening to release private information about a woman onto the Internet in order to get my way is acceptable thing to do. (MD) & .416 \\
\hline It is unacceptable to use the Internet to trace where a woman has been. (ST) & .414 \\
\hline It is acceptable to gain unauthorized access to a woman's information on the Internet. (HC) & .403 \\
\hline Pretending to be someone else when contacting a woman on the Internet is not okay. (IM) & .386 \\
\hline It is unacceptable to bend the truth on the Internet to get the information you want out of a woman. (RC) & .369 \\
\hline Even if I can, I am not free to bypass security to get information about a woman online. (HC) & .361 \\
\hline Just because someone is telling a woman what they think, it does not mean it is threatening or intimidating. (HS) & .339 \\
\hline It is not appropriate to forge identifying information of a woman on the Internet to learn more & .321 \\
\hline
\end{tabular}

Note. $N=276$. Loadings are derived from a single forced factor. $(\mathrm{HC})=$ Hacking; $(\mathrm{IM})=$ Impersonation; $(\mathrm{ST})=$ Surveillance $\&$ Tracking; $(\mathrm{HS})=$ Harassment $\&$ Spamming; $(\mathrm{RC})=$ Recruitment; $(\mathrm{MD})=$ Malicious Distribution. 
Correlations with ideology, threat, sexism, and engagement. Means, standard deviations, skewness, and kurtosis statistics for Study 2 variables are shown in Table 2.2, and zero-order correlations among Study 2 variables are displayed in Table 2.3. After examining skewness values and consulting variable histograms, it was determined that all of the variables showed a negative skew (with the exception of SDO, intergroup anxiety, group dominance threat, hostile sexism and benevolent sexism; i.e. scores were generally low, showing low RWA, perceived threat, and endorsement of and engagement in cyber VAWG); therefore, bootstrapping was performed using 1000 samples. In general, the sociopolitical ideology, perceived threat, sexism, and cyber VAWG measures were significantly and positively inter-correlated, such that greater right-wing ideology, greater perceived threats, greater sexism, and greater endorsement of and engagement in cyber VAWG related to one another, consistent with Hypotheses 1 and 2. Only one anticipated correlation was not significant: benevolent sexism was not significantly correlated with engagement in cyber VAWG. Additionally, realistic threat and group esteem threat were the only threat types to have a significant, albeit small, correlation with benevolent sexism. 
Table 2.2

Means, standard deviations, skewness, kurtosis, minimum, and maximum ${ }^{12}$ values of Study 2 variables

\begin{tabular}{lcccccccccccc}
\hline & RWA & SDO & REAL & SYMB & ANX & EST & SOC & DOM & BS & HS & END & ENG \\
\hline$M$ & 2.27 & 2.75 & 2.60 & 2.40 & 2.91 & 1.74 & 1.59 & 3.09 & 2.50 & 2.71 & 2.15 & 1.18 \\
$S D$ & 1.14 & 1.40 & 2.00 & 2.11 & 1.14 & 1.12 & 1.13 & 1.97 & 1.14 & 1.40 & .93 & .47 \\
Min & 1.00 & 1.00 & 1.00 & 1.00 & 1.00 & 1.00 & 1.00 & 1.00 & 1.00 & 1.00 & 1.00 & 1.00 \\
Max & 7.00 & 7.00 & 10.00 & 10.00 & 6.17 & 7.00 & 7.00 & 7.00 & 5.67 & 5.83 & 7.00 & 6.00 \\
Skewness & 1.07 & .36 & 1.45 & 1.70 & .32 & 1.95 & 2.29 & .59 & .42 & .32 & 1.62 & 5.55 \\
Kurtosis & .99 & -.95 & 1.92 & 2.35 & -.27 & 4.11 & 5.31 & -.96 & -.61 & -1.16 & 4.33 & 43.79
\end{tabular}

Note. $N=276$. RWA = right-wing authoritarianism; $\mathrm{SDO}=$ social dominance orientation; REAL $=$ realistic threat; $\mathrm{SYMB}=$ symbolic threat; $\mathrm{ANX}=$ intergroup anxiety; EST = group esteem threat; $\mathrm{SOC}=$ social threat; $\mathrm{DOM}=$ group dominance threat; $\mathrm{BS}=$ benevolent sexism; $\mathrm{HS}=$ hostile sexism; END = endorsement of cyber VAWG; ENG = engagement in cyber VAWG.

\footnotetext{
${ }^{12}$ Scores for all scales could theoretically range from 1 to 7 , with the exception for realistic threat and symbolic threat which could range from 1 to 10, and engagement in cyber VAWG, and benevolent and hostile sexism which could range from 1 to 6.
} 
Table 2.3

Means, standard deviations, and zero-order correlations between Study 2 variables

\begin{tabular}{|c|c|c|c|c|c|c|c|c|c|c|c|}
\hline Variable & 1 & 2 & 3 & 4 & 5 & 6 & 7 & 8 & 9 & 10 & 11 \\
\hline 1. RWA & - & & & & & & & & & & \\
\hline 2. SDO & $.52 * *$ & - & & & & & & & & & \\
\hline 3. REAL & $.48^{* *}$ & $.52 * *$ & - & & & & & & & & \\
\hline 4. SYMB & $.43 * *$ & $.46^{* *}$ & $.71 * *$ & - & & & & & & & \\
\hline 5. ANX & $.25 * *$ & $.27 * *$ & $.26 * *$ & $.28 * *$ & - & & & & & & \\
\hline 6. EST & $.38 * *$ & $.37 * *$ & $.47 * *$ & $.46^{* *}$ & $.39 * *$ & - & & & & & \\
\hline 7. SOC & $.41 * *$ & $.35 * *$ & $.55^{* *}$ & $.58 * *$ & $.36 * *$ & $.58 * *$ & - & & & & \\
\hline 8. DOM & $.42 * *$ & $.71 *$ & $.48 * *$ & $.45^{* *}$ & $.13^{*}$ & $.25^{* *}$ & $.25 * *$ & - & & & \\
\hline 9. BS & $.37 * *$ & $.18^{* *}$ & $.13^{*}$ & .08 & .05 & $.16^{* *}$ & .12 & .09 & - & & \\
\hline 10. HS & $.53 * *$ & $.72 * *$ & $.63 * *$ & $.54 * *$ & $.32 * *$ & $.39 * *$ & $.41 * *$ & $.68 * *$ & $.37 * *$ & & \\
\hline 11. END & $.46^{* *}$ & $.64 * *$ & $.52 * *$ & $.46^{* *}$ & $.24 * *$ & $.40 * *$ & $.39 * *$ & $.64 * *$ & $.16^{*}$ & $.66^{* *}$ & - \\
\hline 12. ENG & $.28 * *$ & $.31 * *$ & $.38 * *$ & $.38 * *$ & $.13^{*}$ & $.34 * *$ & $.35 * *$ & $.29 * *$ & .05 & $.33 * *$ & $.52 * *$ \\
\hline
\end{tabular}

Note. $N=276$. Bootstrapping with 1000 samples was used with $95 \%$ confidence intervals. $* *$ indicates the correlation is significant at $p<.01, *$ indicates the correlations is significant at $p<$ .05. RWA = right-wing authoritarianism; $\mathrm{SDO}=$ social dominance orientation; $\mathrm{REAL}=$ realistic threat; SYMB = symbolic threat; ANX = intergroup anxiety; EST = group esteem threat; SOC = social threat; DOM = group dominance threat; BS = benevolent sexism; HS = hostile sexism; $\mathrm{END}=$ endorsement of cyber VAWG; ENG = engagement in cyber VAWG. 
Testing the proposed two-stage mediation model. Hypothesis 3, whereby sociopolitical ideologies predict endorsement of and engagement in cyber VAWG via perceived threats and sexist attitudes, was tested using the two path models (i.e. DPM-inspired, ITT-inspired) outlined in the Introduction (see Figures 1 and 2). Path analyses were conducted using AMOS software, based on maximum likelihood procedure and bias-corrected estimates for direct and indirect effects derived from bootstrapping using 1000 samples. Fully saturated models were tested $(d f=0)$, therefore no fit indices are reported. Tables 2.4 and 2.5 summarize the direct and indirect effects for the DPM and ITT-inspired models, respectively.

For the DPM-inspired model, RWA and SDO had significant direct effects on social threat and group dominance threat, respectively, as predicted. SDO also had a significant direct effect on social threat. Together, RWA and SDO explained $19 \%$ and $51 \%$ of the variance in social threat and group dominance threat, respectively. RWA, SDO, social threat, and group dominance threat all had significant direct effects on hostile sexism, whereas only RWA showed a significant direct effect on benevolent sexism. Ideology and threat jointly accounted for $15 \%$ and $62 \%$ of the variance in benevolent and hostile sexism, respectively. The direct paths from SDO, group dominance threat, and hostile sexism to endorsement of cyber VAWG were significant; however, the direct paths from RWA, social threat, and benevolent sexism to endorsement of cyber VAWG were not. Together, ideology, perceived threats, and sexist attitudes explained $54 \%$ of the variance in the endorsement of cyber VAWG. Last, only endorsement of cyber VAWG displayed a significant direct effect on engagement in cyber VAWG, with all predictors cumulatively accounting for $31 \%$ of the variance in engagement in cyber VAWG. 
The indirect effects of RWA and SDO on hostile (but not benevolent) sexism via threat were significant. RWA, SDO, social threat, and group dominance threat all showed significant indirect effects on endorsement of cyber VAWG (via threats and sexism for ideologies, and via sexism for threats). All predictors except for benevolent sexism, mediated by endorsement of cyber VAWG, showed significant indirect effects on engagement in cyber VAWG. Therefore, the proposed model was partially supported, with greater evidence for pathways from SDO, group dominance threat and hostile sexism predicting cyber VAWG. 
Table 2.4

Standardized direct and indirect effects of ideology, DPM threats, and sexist attitudes on endorsement of and engagement in cyber VAWG in Study 2

\begin{tabular}{|c|c|c|c|c|c|c|c|}
\hline \multirow[b]{2}{*}{ Predictors } & \multirow[b]{2}{*}{ Effect } & \multicolumn{6}{|c|}{ Criteria } \\
\hline & & $\mathrm{SOC}$ & DOM & $\mathrm{BS}$ & HS & END & ENG \\
\hline \multirow[t]{2}{*}{ RWA } & Direct & $.31 * *$ & .07 & $.40 * *$ & $.15 * *$ & .07 & .05 \\
\hline & Indirect & & & -.02 & $.07 *$ & $.10 * *$ & $.12 *$ \\
\hline \multirow[t]{2}{*}{ SDO } & Direct & $.19 * *$ & $.67 * *$ & .05 & $.37 * *$ & $.17 *$ & -.04 \\
\hline & Indirect & & & -.07 & $.24 * *$ & $.38 * *$ & $.27 * *$ \\
\hline \multirow[t]{2}{*}{ SOC } & Direct & & & -.04 & $.14^{*}$ & .12 & .17 \\
\hline & Indirect & & & & & $.04 *$ & $.08 * *$ \\
\hline \multirow[t]{2}{*}{ DOM } & Direct & & & -.10 & $.32 * *$ & $.27 * *$ & -.05 \\
\hline & Indirect & & & & & $.10 * *$ & $.19 * *$ \\
\hline \multirow[t]{2}{*}{$\mathrm{BS}$} & Direct & & & & & -.04 & -.05 \\
\hline & Indirect & & & & & & -.02 \\
\hline \multirow[t]{2}{*}{ HS } & Direct & & & & & $.29 * *$ & -.02 \\
\hline & Indirect & & & & & & $.15 * *$ \\
\hline END & Direct & & & & & & $.51 * *$ \\
\hline$R^{2}$ & & .19 & .51 & .15 & .62 & .54 & .31 \\
\hline
\end{tabular}

Note. $N=276 . * *$ indicates the path is significant at $\mathrm{p}<.01$, * indicates the path is significant at $\mathrm{p}<.05 . \mathrm{RWA}=$ right-wing authoritarianism; $\mathrm{SDO}=$ social dominance orientation; $\mathrm{SOC}=$ social threat; DOM = group dominance threat; $\mathrm{BS}=$ benevolent sexism; HS = hostile sexism; $\mathrm{END}=$ endorsement of cyber VAWG; ENG = engagement in cyber VAWG. 
For the ITT-inspired model, RWA and SDO showed significant direct effects on each of realistic threat, symbolic threat, intergroup anxiety, and group esteem threat; with ideology explaining $34 \%, 26 \%, 9 \%$, and $18 \%$ of the variance in the threats respectively. RWA, SDO, realistic threat, and intergroup anxiety all displayed significant direct effects on hostile sexism, whereas the only direct effect observed on benevolent sexism was from RWA. Ideology and threat combined accounted for $15 \%$ and $63 \%$ of the variance in benevolent and hostile sexism, respectively. Significant direct effects on endorsement of cyber VAWG were observed from only SDO and hostile sexism, with ideology, threat, and sexist attitudes together explaining 52\% of the variance in endorsement of cyber VAWG. Only endorsement of cyber VAWG showed a significant direct effect on engagement in cyber VAWG, with all predictors cumulatively explaining $32 \%$ of the variance in engagement in cyber VAWG.

Similar to the previous model, significant indirect effects of RWA and SDO, via threats, were observed for hostile sexism, but no significant paths were observed for benevolent sexism. However, significant indirect effects on endorsement of cyber VAWG were observed for both ideologies, realistic threat, symbolic threat, and intergroup anxiety, via threats and sexism for ideologies and via sexism for threats. Finally, RWA, SDO, and hostile sexism showed significant indirect effects on engagement in cyber VAWG, with paths from ideologies mediated by threats and sexism and paths from hostile sexism mediated by threats. Therefore, partial support for the proposed model was found, with RWA additionally showing indirect effects on endorsement of and engagement in cyber VAWG via threats based on ITT. 
Table 2.5

Standardized direct and indirect effects of ideology, non-DPM threats, and sexist attitudes on endorsement of and engagement in cyber VAWG in Study 2

\begin{tabular}{|c|c|c|c|c|c|c|c|c|c|}
\hline \multirow[b]{2}{*}{ Predictors } & \multirow[b]{2}{*}{ Effect } & \multicolumn{8}{|l|}{ Criteria } \\
\hline & & REAL & SYMB & ANX & EST & BS & HS & END & ENG \\
\hline \multirow[t]{2}{*}{ RWA } & Direct & $.29 * *$ & $.26 * *$ & $.16^{*}$ & $.26 * *$ & $.40 * *$ & $.11^{*}$ & .08 & .03 \\
\hline & Indirect & & & & & -.02 & $.10^{* *}$ & $.10 * *$ & $.14 * *$ \\
\hline \multirow[t]{2}{*}{ SDO } & Direct & $.37 * *$ & $.33 * *$ & $.18 * *$ & $.24 * *$ & .01 & $.48 * *$ & $.26 * *$ & -.08 \\
\hline & Indirect & & & & & -.03 & $.13^{* *}$ & $.28 * *$ & $.30 * *$ \\
\hline \multirow[t]{2}{*}{ REAL } & Direct & & & & & -.01 & $.26 * *$ & .04 & .08 \\
\hline & Indirect & & & & & & & $.09 * *$ & .04 \\
\hline \multirow[t]{2}{*}{ SYMB } & Direct & & & & & -.11 & .07 & .04 & .13 \\
\hline & Indirect & & & & & & & $.04 *$ & .03 \\
\hline \multirow[t]{2}{*}{ ANX } & Direct & & & & & -.06 & $.09 *$ & -.04 & -.05 \\
\hline & Indirect & & & & & & & $.04 * *$ & -.01 \\
\hline \multirow[t]{2}{*}{ EST } & Direct & & & & & .08 & -.02 & .11 & .13 \\
\hline & Indirect & & & & & & & -.01 & .05 \\
\hline \multirow[t]{2}{*}{ BS } & Direct & & & & & & & -.08 & -.03 \\
\hline & Indirect & & & & & & & & -.04 \\
\hline \multirow[t]{2}{*}{ HS } & Direct & & & & & & & $.38 * *$ & -.09 \\
\hline & Indirect & & & & & & & & $.19 * *$ \\
\hline END & Direct & & & & & & & & $.48 * *$ \\
\hline$R^{2}$ & & .34 & .26 & .09 & .18 & .15 & .63 & .52 & .32 \\
\hline
\end{tabular}

Note. $N=276 . * *$ indicates the path is significant at $\mathrm{p}<.01, *$ indicates the path is significant at $\mathrm{p}<.05 . \mathrm{RWA}=$ right-wing authoritarianism; $\mathrm{SDO}=$ social dominance orientation; $\mathrm{REAL}=$ realistic threat; SYMB = symbolic threat; ANX = intergroup anxiety; EST = group esteem threat; $\mathrm{BS}=$ benevolent sexism; HS = hostile sexism; END = endorsement of cyber VAWG; ENG = engagement in cyber VAWG. 


\section{Discussion}

In Study 2 all three hypotheses were evaluated using a more representative, online sample of gamers. Of note, the majority (87\%) of participants in Study 2 were male. Hence, the results reported here are applicable to the population of interest: male gamers. As in Study 1, factor analyses identified a unidimensional construct for attitudes towards cyber VAWG, despite items reflecting six distinct domains of behaviour as categorized by The Learning Network and the United Nations' report on cyber VAWG (Baker et al., 2013; United Nations Entity for Gender Equality and the Empowerment of Women and United Nations Development Programme, 2015). Similar to the factor loadings found for the scale in Study 1 (see Table 1.1), harassment and spamming, and recruitment items loaded most strongly onto the factor, with malicious distribution and impersonation items also loading more strongly relative to items pertaining to surveillance, and tracking and hacking. Consistent with Hypothesis 1, right-wing ideology, perceived threat, and sexism related to endorsement of and engagement in cyber VAWG, with the exception that benevolent sexism did not relate to engagement in cyber VAWG. As discussed in Study 1, where benevolent sexism also failed to show a relation with cyber VAWG outcomes, the lack of a significant relation may reflect the incompatibility of the subjectively positive attitudes and behaviours of benevolent sexism with the intentionally harmful and manipulative nature of cyber VAWG behaviours (Glick \& Fiske, 1996).

RWA and SDO related to all types of perceived threats and sexist attitudes (Hypothesis 2). This is largely consistent with literature on the relations of RWA (Charles-Toussaint \& Crowson, 2010; Ruffman et al., 2016) and SDO with threats described in ITT. (Bustillos \& Silván-ferrero, 2013; Crowson, 2009; Uenal, 2016) Conversely, RWA and SDO did not differentially relate to ambivalent sexist attitudes and DPM threats (i.e. social threat, group 
dominance threat) as they have elsewhere (Duckitt \& Sibley, 2009; Sibley et al., 2007). Further, RWA even showed stronger relations with hostile (vs. benevolent) sexism and social (vs. group dominance) threat. It is possible that these additional unexpected associations are attributable to the present sample of online gamers and cyber VAWG, in particular. That is, RWA may be relating to the threats that are simply more relevant in this context.

Support was found for both mediation models described in Hypothesis 3. With respect to the DPM-inspired model, RWA and SDO predicted social and group dominance threats respectively, as expected (Duckitt, 2001). However, unexpectedly, SDO additionally predicted social threat. Also consistent with the DPM and previous literature (Sibley et al., 2007), RWA and SDO predicted benevolent and hostile sexism, respectively. RWA unexpectedly also predicted hostile sexism, but this relation was weaker than its relation with benevolent sexism. Social and group dominance threat predicted hostile, but not benevolent sexism, again suggesting the relevance of hostile over benevolent sexism for attitudes toward female gamers. Right-wing ideology, perceived threats (most reliably social threat and group dominance threat), and hostile (but not benevolent) sexism predicted endorsement of and engagement in cyber VAWG. Last, endorsement of cyber VAWG predicted engagement in cyber VAWG. Therefore, hostile sexist attitudes and perceived threats of female gamers helped explain the relation between right-wing ideology and attitudes towards and participation in cyber VAWG-related behaviour.

In the ITT-inspired model, RWA and SDO predicted each of the perceived threats of female gamers, with the strongest associations with realistic and symbolic threats, similar to the results of Study 1. Further, consistent with the literature (Sibley et al., 2007), RWA predicted benevolent sexism and SDO predicted hostile sexism. Inconsistent with research and theory (Duckitt, 2001; Sibley et al., 2007), RWA additionally predicted hostile sexism - but as noted 
above, this relation was weaker than with benevolent sexism. Perceiving female gamers as threatening to acquire resources and as being awkward to interact with predicted hostile sexism. In contrast, none of the perceived threats predicted benevolent sexism. RWA, SDO, realistic threat, symbolic threat, intergroup anxiety, and hostile sexism predicted endorsement of cyber VAWG. RWA, SDO, and hostile (but not benevolent) sexism, predicted engagement in cyber VAWG. Last, endorsement of cyber VAWG predicted engagement in cyber VAWG. As in the DPM-inspired model, the relation between right-wing ideology and cyber VAWG was mediated by greater perceived threats of competition for resources and awkward interactions with female gamers and hostile sexist attitudes.

Across both models, ideology (RWA, SDO), mediated by perceived threats (specifically social threat, group dominance threat, realistic threat, and intergroup anxiety) and hostile sexism, consistently predicted endorsement of and engagement in cyber VAWG. Furthermore, endorsement of cyber VAWG consistently predicted engagement in cyber VAWG. Notably, benevolent sexism did not predict either endorsement of, or engagement in, cyber VAWG. This suggests that the relation between sexism and cyber VAWG may be purely antipathic and not reflect any of the subjectively positive beliefs that underlie benevolent sexism (Glick \& Fiske, 1996). Altogether, these findings provide support for the third hypothesis and the presence of mediation at several stages, with greater RWA, SDO, and hostile sexism emerging as primary theoretical antecedents of, and the most relevant psychological constructs in, understanding cyber VAWG. The relations observed in both models are largely consistent with the differential effects and differential mediation hypotheses postulated by the DPM (Duckitt \& Sibley, 2009). 


\section{Study 3}

As explained above, Study 3 consisted of participants from the sample originally collected for Study 2. Specifically, Study 3 comprised participants who completed the survey after a widely-viewed livestreamed and posted video of two individuals completing the survey and discussing the survey was posted. Hence, Study 3 participants might have been, and likely were, influenced by the posted video. Although the purpose of Study 3 was to test the study hypotheses, the results of Study 3 must be interpreted with caution and in consideration of the biased impression many participants may have had when completing the study.

\section{Methods}

Participants and procedure. A total of 13,581 completed responses were recorded after the survey went viral. Participants were removed from the original "post viral" sample for: not consenting to participate (2929), incorrectly answering "dummy" questions designed to screen for automated responding (1361), withdrawing part-way through the study (2232), opting out of having their responses included (74), taking less than 10 minutes or longer than 3 hours to complete (155), having duplicate responses from the same computer (35), demonstrating acquiescent responding on more than one scale (181), and missing a significant number of responses for items (233). This left a sample of 6381 participants $\left(M_{\mathrm{age}}=24.12, S D=6.49\right.$; Gender - Male: 90.7\%, Female: 5.3\%, Other: 4.0\%; Country - United States: 52.7\%, United

Kingdom $11.8 \%$, Canada $8.0 \%$, Germany $3.3 \%$, Brazil 0.8\%, France $0.6 \%$, Russia $0.6 \%$, Japan $0.5 \%$, Mexico $0.4 \%$, Spain $0.4 \%$, South Korea $0.1 \%$, China $0.05 \%$, Other $20.8 \%$; Ethnicity White/Caucasian 73.2\%, Latin American 3.5\%, Black 2.2\%, Chinese 0.7\%, Arab/West Asian 0.6\%, South Asian 0.6\%, Filipino 0.4\%, Southeast Asian 0.4\%, Japanese 0.2\%, Korean 0.1\%, Other 7.4\%, 10.8\% Multiethnic). 
Measures. The procedures and measures administered in Study 3 were identical to those described in Study 2. Participants completed: a 12-item version of the RWA scale $(\alpha=.85$; Altemeyer, 1981, 1988, 1996, 2006), an 8 -item version of the $\mathrm{SDO}_{7}$ scale ( $\alpha=.67$; Ho et al., 2015), a 2-item modified realistic threat scale ( $r=.46$; Stephan \& Stephan, 2000; Stephan et al., 1999), a 2-item modified symbolic threat scale ( $r=.66$; Stephan \& Stephan, 2000; Stephan et al., 1999), a 6-item intergroup anxiety scale ( $\alpha=.73$; Stephan $\&$ Stephan, 1985), a 2-item group esteem threat scale ( $r=.45$; CSES-R; Luhtanen \& Crocker, 1992), a 2-item modified social threat scale ( $r=.70$; Charlesford \& Choma, 2013; Choma et al., 2016), a 2-item modified group dominance threat scale ( $r=.24$; Charlesford \& Choma, 2013; Choma et al., 2016), a 12-item version of the ASI (hostile sexism $\alpha=.82$; benevolent sexism $\alpha=.78$; Glick \& Fiske, 1996; Rollero, Glick, \& Tartaglia, 2014), a 46-item measure of endorsement of cyber VAWG, and a 6item measure of engagement in cyber VAWG $(\alpha=.74)$. Three items assessing group esteem threat were administered, however only the two most reliable were included in the computation of the group esteem threat score (see Appendix H).

\section{Results}

Cyber VAWG endorsement scale development. As in Studies 1 and 2, PAF was conducted on the 36 targeted items assessing endorsement of cyber VAWG, applying an oblique rotation (i.e., promax, with kappa set to 4). Seven factors emerged with eigenvalues greater than 1 (8.90, 2.26, 1.82, 1.50, 1.13, 1.10, 1.03; see Appendix L for a table of factor loadings). Examination of the scree plot, shown in Figure 5, suggested extraction of a single factor that accounts for $24.72 \%$ of the variance. As the items loaded predominantly onto one factor (Factor 1) representing support of cyber VAWG, another PAF forcing the extraction of one factor was conducted. The loadings for each item on the single forced extracted factor are shown in Table 
3.1. Of the 36 items, 33 loaded stronger than .32 onto the single extracted factor. The 3 items that did not load stronger than .32 were dropped (see Costello \& Osborne, 2005). Thus, the 33 items with loadings stronger than .32 were selected and averaged in the computation of the endorsement of cyber VAWG scale $(\alpha=.90)$. Higher scores on the scale indicated greater endorsement of cyber VAWG.

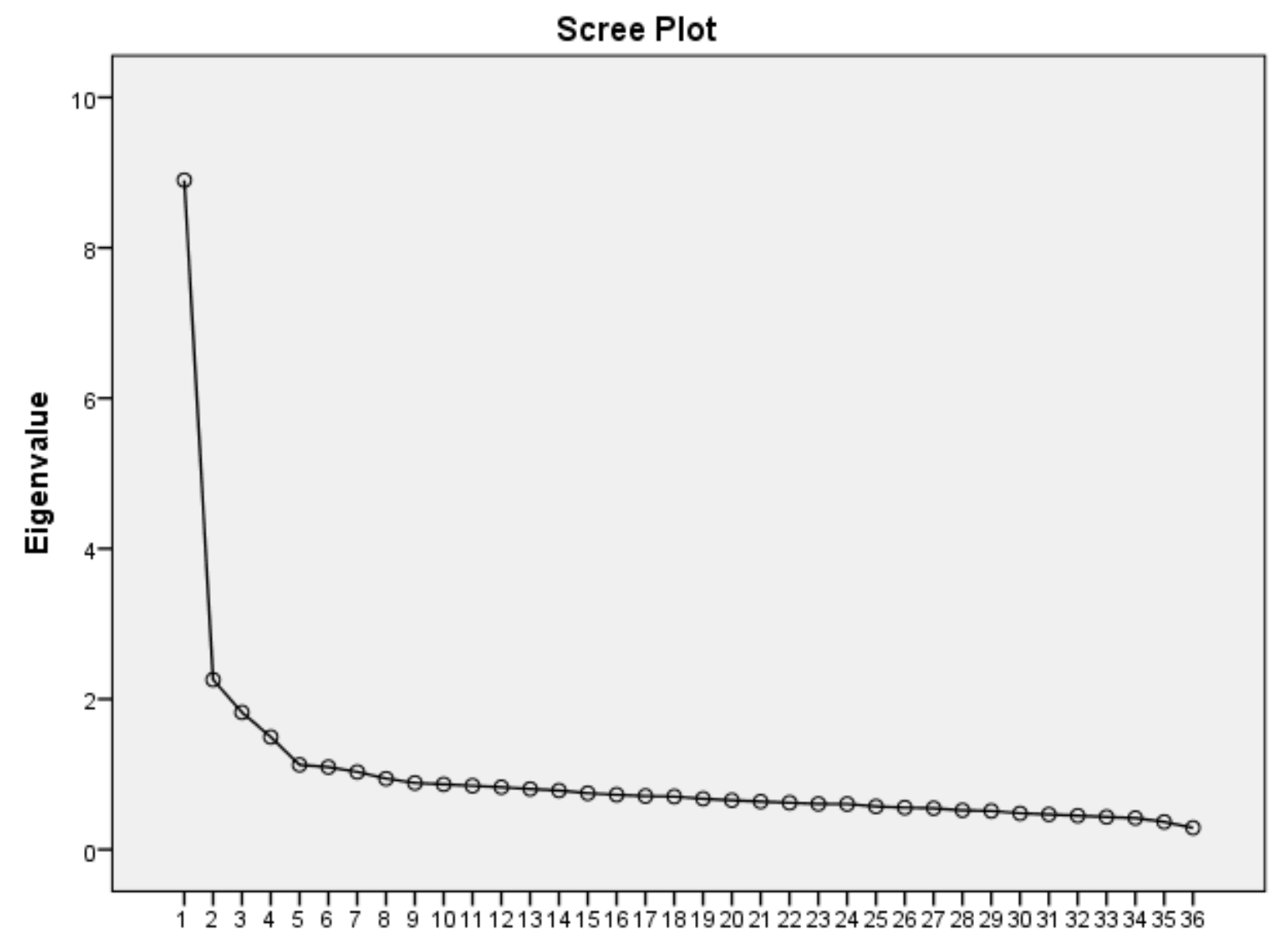

Factor Number

Figure 5. Scree plot produced from principal axis factoring of items assessing endorsement of cyber VAWG in Study 3. 
Table 3.1

Loadings of Cyber VAWG items on the single forced factor in Study 3

\begin{tabular}{|c|c|}
\hline Item & Loading \\
\hline It is okay to trick a woman into a compromising situation using the Internet. (RC) & .670 \\
\hline There is nothing wrong with using fake advertisements or profiles to trick a woman online. (RC) & 670 \\
\hline $\begin{array}{l}\text { It's okay to create new, anonymous online profiles or accounts for the sole purpose of using them to spam a woman with messag es. } \\
\text { (HS) }\end{array}$ & .652 \\
\hline It is okay to mislead a woman online. (RC) & .620 \\
\hline There's nothing wrong with making unprovoked sexual remarks towards or sexual requests of a woman on the Internet. (HS) & .613 \\
\hline It is okay to impersonate someone online to learn more about a woman. (IM) & .607 \\
\hline To get information about a woman, it's okay to pose as her online. (IM) & .598 \\
\hline If I have figured out the password to a woman's account or profile, I can do what I want with it. (IM) & .583 \\
\hline It is okay to share intimate photos or videos of a woman online without her consent. (MD) & .575 \\
\hline It is fine to use the Internet to contact a woman even though she does not want to be contacted. (HS) & .528 \\
\hline Continually monitoring the activities of a woman online is totally fine. (ST) & .527 \\
\hline Spamming a woman's e-mail, social media, phone or voicemail with messages is wrong. (HS) & .502 \\
\hline On the Internet, to convince a woman of something, sometimes you have to say things that are not entirely true. (RC) & .484 \\
\hline It is unacceptable to use the Internet to trace where a woman has been. (ST) & .482 \\
\hline There's nothing wrong with threatening real life violence to a woman on the Internet. (HS) & .476 \\
\hline Twisting the truth about a woman online for my personal purposes is not right. (MD) & .467 \\
\hline I'm at liberty to send intimate photos of myself to any woman I choose. (MD) & .466 \\
\hline There is no problem with telling a woman to kill herself on the Internet. (HS) & .455 \\
\hline Obtaining a woman's personal information online without her permission is never okay. $(\mathrm{HC})$ & .443 \\
\hline It is not okay to use the Internet to track down a woman. (ST) & .440 \\
\hline There is nothing wrong with using the Internet to trace a woman's history. (ST) & .436 \\
\hline If I want to access information about a woman online, I can do what I need to do to acquire it. (HC) & .423 \\
\hline I do not have the right to spread fake stories or rumors about a woman online. (MD) & .415 \\
\hline It is okay to use the Internet to tell a woman what I think about her, regardless of if she wants to know or not. (HS) & .410 \\
\hline It is acceptable to gain unauthorized access to a woman's information on the Internet. (HC) & .399 \\
\hline It is okay to gain access to a live stream of a woman's webcam without her knowledge. (HC) & .399 \\
\hline It is not okay to use GPS or location tracking to find out where a woman is right now. (ST) & .389 \\
\hline Pretending to be someone else when contacting a woman on the Internet is not okay. (IM) & .388 \\
\hline There is potential harm in repeatedly messaging, calling or texting a woman. (HS) & .387 \\
\hline Even if I can, I am not free to bypass security to get information about a woman online. (HC) & .385 \\
\hline I have the right to express my anger towards a woman online as frequently as I wish. (HS) & .345 \\
\hline It is not appropriate to forge identifying information of a woman on the Internet to learn more about her. (IM) & .332 \\
\hline Threatening to release private information about a woman onto the Internet in order to get my way is acceptable thing to do. (MD) & .324 \\
\hline It is unacceptable to bend the truth on the Internet to get the information you want out of a woman. (RC) & .315 \\
\hline If a woman has an online presence, it is okay for me to use information she puts out there to learn more about her. (ST) & .302 \\
\hline Just because someone is telling a woman what they think, it does not $\mathrm{r}$ & .106 \\
\hline
\end{tabular}

Note. $N=6381$. Loadings are derived from forced extraction onto a single factor. $(\mathrm{HC})=$ Hacking; $(\mathrm{IM})=$ Impersonation; $(\mathrm{ST})=$ Surveillance \& Tracking; $(\mathrm{HS})=$ Harassment \& Spamming; $(\mathrm{RC})=$ Recruitment $(\mathrm{MD})=$ Malicious Distribution. 
Correlations with ideology, threat, sexism, and engagement. Means, standard deviations, skewness, and kurtosis statistics for Study 3 variables are shown in Table 3.2, and zero-order correlations among Study 3 variables are displayed in Table 3.3. Upon examining skewness values and consulting variable histograms, symbolic threat, group esteem threat, social threat, and endorsement of and engagement in cyber VAWG showed a negative skew (i.e. scores were generally low, showing lower perceived threats and endorsement of and engagement in cyber VAWG); therefore, bootstrapping was performed using 1000 samples. Further, adjusted 99\% (vs. 95\%) confidence intervals were used in computations to account for the large sample size. Due to the large sample size, the analyses conducted for Study 3 had considerable power, and are more likely to produce significant correlations which are difficult to interpret, as seen in Table 3.3 where the majority of the correlations are significant at the $p<.01$ level. In lieu of this, a +/-.10 magnitude threshold for correlations and estimates to distinguish meaningful results was implemented. In general, the ideology, threat, sexism, and cyber VAWG variables were significantly and positively inter-correlated, largely providing support for Hypotheses 1 and 2, with a few notable exceptions: group dominance threat was unrelated to engagement in cyber VAWG, and benevolent sexism was unrelated to endorsement of and engagement in cyber VAWG. 
Table 3.2

Means, standard deviations, skewness, kurtosis, minimum, and maximum ${ }^{13}$ values of Study 3 variables

\begin{tabular}{lcccccccccccc}
\hline & RWA & SDO & REAL & SYMB & ANX & EST & SOC & DOM & BS & HS & END & ENG \\
\hline$M$ & 2.75 & 3.76 & 3.60 & 3.29 & 3.21 & 1.64 & 1.85 & 4.96 & 2.56 & 3.79 & 2.47 & 1.21 \\
SD & 1.06 & 1.02 & 2.13 & 2.39 & 1.05 & .97 & 1.25 & 1.18 & 1.00 & .96 & .82 & .45 \\
Min & 1.00 & 1.00 & 1.00 & 1.00 & 1.00 & 1.00 & 1.00 & 1.00 & 1.00 & 1.00 & 1.00 & 1.00 \\
Max & 7.00 & 7.00 & 10.00 & 10.00 & 7.00 & 7.00 & 7.00 & 7.00 & 6.00 & 6.00 & 7.00 & 6.00 \\
Skewness & .70 & .08 & .70 & .91 & -.03 & 1.48 & 1.75 & -.33 & .35 & -.17 & 1.14 & 5.11 \\
Kurtosis & .41 & .27 & .12 & .03 & -.29 & 3.23 & 2.86 & .14 & -.45 & -.09 & 2.74 & 40.65 \\
& & & & & & & & & & & & \\
\hline
\end{tabular}

Note. $N=6381$. $\mathrm{RWA}=$ right-wing authoritarianism; $\mathrm{SDO}=$ social dominance orientation; REAL = realistic threat; SYMB = symbolic threat; ANX = intergroup anxiety; EST = group esteem threat; $\mathrm{SOC}=$ social threat DOM = group dominance threat $\mathrm{HS}=$ hostile sexism; $\mathrm{BS}=$ benevolent sexism; END = endorsement of cyber VAWG; ENG = engagement in cyber VAWG.

\footnotetext{
${ }^{13}$ Scores for all scales could theoretically range from 1 to 7 , with the exception for realistic threat and symbolic threat which could range from 1 to 10, and engagement in cyber VAWG, and benevolent and hostile sexism which could range from 1 to 6.
} 
Table 3.3

Means, standard deviations, and zero-order correlations between Study 3 variables

\begin{tabular}{|c|c|c|c|c|c|c|c|c|c|c|c|}
\hline Variable & 1 & 2 & 3 & 4 & 5 & 6 & 7 & 8 & 9 & 10 & 11 \\
\hline 1. RWA & - & & & & & & & & & & \\
\hline 2. SDO & $.43 *$ & - & & & & & & & & & \\
\hline 3. REAL & $.34 *$ & $.31 *$ & - & & & & & & & & \\
\hline 4. SYMB & $.33 *$ & $.28^{*}$ & $.71 *$ & - & & & & & & & \\
\hline 5. ANX & $.21 *$ & $.16^{*}$ & $.28 *$ & $.29 *$ & - & & & & & & \\
\hline 6. EST & $.32 *$ & $.24^{*}$ & $.34 *$ & $.35^{*}$ & $.31 *$ & - & & & & & \\
\hline 7. SOC & $.38 *$ & $.30^{*}$ & $.52 *$ & $.57 *$ & $.33^{*}$ & $.54^{*}$ & - & & & & \\
\hline 8. DOM & $.13 *$ & $.29 *$ & $.18^{*}$ & $.12 *$ & .06 & .06 & .09 & - & & & \\
\hline 9. BS & $.38 *$ & $.15^{*}$ & $.11 *$ & $.10 *$ & .03 & .09 & $.10 *$ & $-.13 *$ & - & & \\
\hline 10. HS & $.38 *$ & $.38^{*}$ & $.47^{*}$ & $.43 *$ & $.25^{*}$ & $.24^{*}$ & $.39 *$ & $.26^{*}$ & $.19^{*}$ & - & \\
\hline 11. END & $.29 *$ & $.35^{*}$ & $.32 *$ & $.30 *$ & $.21 *$ & $.30 *$ & $.34 *$ & $.31 *$ & $<.01$ & $.37 *$ & - \\
\hline 12. ENG & $.19 *$ & $.18^{*}$ & $.19 *$ & $.18 *$ & $.13 *$ & $.23^{*}$ & $.21 *$ & .07 & .04 & $.19 *$ & $.42 *$ \\
\hline
\end{tabular}

Note. $N=6381$. Bootstrapping with 1000 samples was used with $99 \%$ confidence intervals. $*$ indicates a correlation with a magnitude greater than .10 that is significant at $p<.05$. RWA $=$ right-wing authoritarianism; $\mathrm{SDO}=$ social dominance orientation; REAL = realistic threat; SYMB = symbolic threat; ANX = intergroup anxiety; EST = group esteem threat; $\mathrm{SOC}=$ social threat; DOM = group dominance threat; HS = hostile sexism; BS = benevolent sexism; $\mathrm{END}=$ endorsement of cyber VAWG; ENG = engagement in cyber VAWG. 
Testing the proposed two-stage mediation model. To test hypothesis 3 (see Figures 1

and 2), path analyses were again conducted using AMOS software, based on maximum likelihood procedure and bias-corrected estimates for direct and indirect effects derived from bootstrapping using 1000 samples. Fully saturated models were tested $(d f=0)$, therefore no fit indices are report. Tables 3.4 and 3.5 summarize the direct and indirect effects for the DPM and ITT-inspired models, respectively.

For the DPM-inspired model, RWA and SDO each had a significant direct on social threat, however only SDO had a significant direct effect on group dominance threat. Ideology accounted for $17 \%$ and $9 \%$ of the variance in social threat and group dominance threat respectively. RWA and group dominance threat had significant direct effects on benevolent sexism and hostile sexism, with SDO and social threat only having a significant direct effect on hostile sexism. As a result, ideology and threat accounted for $18 \%$ and $28 \%$ of the variance in benevolent and hostile sexism respectively. SDO, social threat, group dominance threat, and hostile sexism all had significant direct effects on endorsement of cyber VAWG, whereas only endorsement of cyber VAWG had a significant direct effect on engagement in cyber VAWG. Notably, less (vs. greater, as expected) group dominance threat predicted greater benevolent sexism. Thus, ideology, threat, and sexist attitudes accounted for $27 \%$ of the variance in endorsement of cyber VAWG, and all predictors accounted for $19 \%$ of the variance in engagement in cyber VAWG. The only significant indirect effects observed were that of SDO on endorsement of and engagement in cyber VAWG. Therefore, partial evidence of mediation was found, principally through pathways originating from SDO to cyber VAWG outcomes. 
Table 3.4

Standardized direct and indirect effects of ideology, DPM threats, and sexist attitudes on endorsement of and engagement in cyber VAWG in Study 3

\begin{tabular}{|c|c|c|c|c|c|c|c|}
\hline \multirow[b]{2}{*}{ Predictors } & \multirow[b]{2}{*}{ Effect } & \multicolumn{6}{|c|}{ Criteria } \\
\hline & & SOC & $\mathrm{DOM}$ & BS & HS & END & ENG \\
\hline \multirow[t]{2}{*}{ RWA } & Direct & $.31^{*}$ & .01 & $.41 *$ & $.19^{*}$ & $.10^{14}$ & .05 \\
\hline & Indirect & & & -.02 & .08 & .08 & .09 \\
\hline \multirow[t]{2}{*}{ SDO } & Direct & $.17 *$ & $.29 *$ & .05 & $.18^{*}$ & $.15^{*}$ & .02 \\
\hline & Indirect & & & -.07 & .09 & $.13^{*}$ & $.10^{*}$ \\
\hline \multirow[t]{2}{*}{ SOC } & Direct & & & -.05 & $.25^{*}$ & $.19 *$ & .05 \\
\hline & Indirect & & & & & .05 & $.10^{15}$ \\
\hline \multirow[t]{2}{*}{ DOM } & Direct & & & $-.20 *$ & $.16^{*}$ & $.18 *$ & -.08 \\
\hline & Indirect & & & & & .05 & .09 \\
\hline \multirow[t]{2}{*}{ BS } & Direct & & & & & -.08 & $<.01$ \\
\hline & Indirect & & & & & & -.03 \\
\hline \multirow[t]{2}{*}{ HS } & Direct & & & & & $.18 *$ & .01 \\
\hline & Indirect & & & & & & .07 \\
\hline END & Direct & & & & & & $.40^{*}$ \\
\hline$R^{2}$ & & .17 & .09 & .18 & .28 & .27 & .19 \\
\hline
\end{tabular}

Note. $N=6381 . *$ indicates a correlation with a magnitude greater than .10 that is significant at $\mathrm{p}$ $<.01$. RWA = right-wing authoritarianism; $\mathrm{SDO}=$ social dominance orientation; $\mathrm{SOC}=$ social threat; DOM = group dominance threat; HS = hostile sexism; $\mathrm{BS}=$ benevolent sexism; $\mathrm{END}=$ endorsement of cyber VAWG; ENG = engagement in cyber VAWG.

\footnotetext{
${ }^{14}$ Non-rounded value is .096 , thus this was not flagged as significant.

15 Non-rounded value is .097 , thus this was not flagged as significant.
} 
For the ITT-inspired model, RWA and SDO had significant direct effects on all four threat types (except SDO on intergroup anxiety), together accounting for $15 \%, 13 \%, 5 \%$, and $14 \%$ of the variance in realistic threat, symbolic threat, intergroup anxiety, and group esteem threat respectively. Only RWA had a significant direct effect on benevolent sexism, whereas both ideologies, realistic threat, and symbolic threat all had significant direct effects on hostile sexism. Ideology and threat variables together explained $15 \%$ and $32 \%$ variance in benevolent and hostile sexism respectively. Ideologies, threats, and ambivalent sexist attitudes cumulatively accounted for $25 \%$ of the variance in cyber VAWG, however the only direct effects observed were from SDO, and benevolent and hostile sexism. Notably, the direct pathway between benevolent sexism and endorsement of cyber VAWG was in the opposite direction than anticipated, such that greater benevolent sexism predicted less supportive attitudes towards cyber VAWG behaviours - this effect was very small $(-.12, p=.002)$. Only group esteem threat and endorsement of cyber VAWG had significant direct effects on engagement in cyber VAWG. Altogether, all predictor variables cumulatively explained $19 \%$ of the variance in engagement in cyber VAWG.

The only significant indirect effect of ideologies on ambivalent sexist attitudes observed was that of RWA on hostile sexism. No indirect effects on endorsement of cyber VAWG were observed. Only SDO showed a significant indirect effects on engagement in cyber VAWG. Therefore, the ITT-inspired model showed considerable evidence of threat and sexism mediating the relation between ideologies and endorsement of cyber VAWG, and endorsement of cyber VAWG functioning as an additional mediator between predictors and engagement in cyber VAWG. 
Table 3.5

Standardized direct and indirect effects of ideology, non-DPM threats, and sexist attitudes on endorsement of and engagement in cyber VAWG in Study 3

\begin{tabular}{|c|c|c|c|c|c|c|c|c|c|}
\hline \multirow[b]{2}{*}{ Predictors } & \multirow[b]{2}{*}{ Effect } & \multicolumn{8}{|l|}{ Criteria } \\
\hline & & REAL & SYMB & ANX & EST & BS & HS & END & ENG \\
\hline \multirow[t]{2}{*}{ RWA } & Direct & $.26^{*}$ & $.25^{*}$ & $.17 *$ & $.29 *$ & $.40 *$ & $.15^{*}$ & .09 & .04 \\
\hline & Indirect & & & & & -.02 & $.12^{*}$ & .08 & $.10^{16}$ \\
\hline \multirow[t]{2}{*}{ SDO } & Direct & $.20 *$ & $.17 *$ & .09 & $.14 *$ & -.01 & $.18^{*}$ & $.19 *$ & .01 \\
\hline & Indirect & & & & & -.01 & .08 & .09 & $.12 *$ \\
\hline \multirow[t]{2}{*}{ REAL } & Direct & & & & & $<-.01$ & $.24 *$ & .05 & .02 \\
\hline & Indirect & & & & & & & .05 & .04 \\
\hline \multirow[t]{2}{*}{ SYMB } & Direct & & & & & $<-.01$ & $.13^{*}$ & .02 & $<.01$ \\
\hline & Indirect & & & & & & & .03 & .02 \\
\hline \multirow[t]{2}{*}{ ANX } & Direct & & & & & -.05 & .08 & .04 & .02 \\
\hline & Indirect & & & & & & & .02 & .02 \\
\hline \multirow[t]{2}{*}{ EST } & Direct & & & & & -.03 & .03 & $.16^{*}$ & .08 \\
\hline & Indirect & & & & & & & .01 & .06 \\
\hline \multirow[t]{2}{*}{ BS } & Direct & & & & & & & $-.12 *$ & .01 \\
\hline & Indirect & & & & & & & & -.05 \\
\hline \multirow[t]{2}{*}{ HS } & Direct & & & & & & & $.20^{*}$ & -.01 \\
\hline & Indirect & & & & & & & & .07 \\
\hline END & Direct & & & & & & & & $.37 *$ \\
\hline$R^{2}$ & & .15 & .13 & .05 & .14 & .15 & .32 & .25 & .19 \\
\hline
\end{tabular}

Note. $N=6381 . *$ indicates a correlation with a magnitude greater than .10. RWA $=$ right-wing authoritarianism; $\mathrm{SDO}=$ social dominance orientation; REAL = realistic threat; SYMB = symbolic threat; ANX = intergroup anxiety; EST = group esteem threat; HS = hostile sexism; BS = benevolent sexism; END = endorsement of cyber VAWG; ENG = engagement in cyber VAWG.

${ }^{16}$ Non-rounded value is .099 , thus this was not flagged as significant. 


\section{Discussion}

Study 3 tested all three hypotheses in a large representative sample of online gamers, the majority (90.7\%) of which were men. Circumstantially, the responses in Study 3 may reflect the unpredictable influence from viewing the livestream or archived video, and results must be interpreted with caution. As in the previous two studies, factor analyses suggested a single factor (Factor 1) representing attitudes towards cyber VAWG, despite the measure containing items that reflect six different subdomains of cyber VAWG behaviour (see Baker et al., 2013; United Nations Entity for Gender Equality and the Empowerment of Women and United Nations Development Programme, 2015).

Right-wing ideology, perceived threat, and hostile, but not benevolent sexism related to endorsement of, and engagement in, cyber VAWG, with the exception of group esteem being unrelated to engagement in cyber VAWG (i.e. Hypothesis 1). The lack of association between benevolent sexism and cyber VAWG outcomes replicates the findings of Studies 1 and 2 . As noted above, this finding is not surprising given that the subjectively positive nature of benevolent sexist attitudes is in opposition with the intentionally harmful nature of the majority of attitudes and behaviours that comprise cyber VAWG (Glick \& Fiske, 1996).

RWA and SDO related to all types of perceived threats and sexism, as found in Study 2 (Hypothesis 2). As every possible relation of ideology with threat and sexism was positive and significant, the results are simultaneously consistent and inconsistent with the DPM (Duckitt, 2001), as the DPM specifies that threats are exclusively related to their respective ideologies (i.e. social threat with RWA, group dominance threat with SDO). Further, whereas previous literature on ambivalent sexist attitudes shows that RWA uniquely relates to benevolent sexism and SDO uniquely relates to hostile sexism (Duckitt \& Sibley, 2007), the present study found that RWA 
and SDO related to both types of sexism. SDO, however, was more strongly related with hostile versus benevolent sexism, as would be expected.

Partial support was found for Hypothesis 3. With respect to the DPM-inspired model (Duckitt, 2001), RWA and SDO predicted social threat and group dominance threat, respectively. Inconsistent with the DPM, SDO also predicted social threat - though this path was not as strong as the path with group dominance. As expected, RWA and SDO predicted benevolent and hostile sexism, respectively. Consistent with the results of Study 2, but contrary to the literature (Sibley et al., 2007), RWA additionally predicted hostile sexism with similar strength to that of SDO predicting hostile sexism. Unlike in Study 2, where SDO predicted hostile sexism much stronger than RWA did, RWA and SDO predicted hostile sexism with similar strength in Study 3. Perceiving females as being threatening to traditional gamer social structures predicted hostile, but not benevolent sexism. Oddly, and unique to Study 3, less group dominance threat predicted greater benevolent sexism. It is possible that if gamers are not threatened that female gamers will dominate over male gamers, they are more likely to react positively instead of with hostility, opening the possibility for benevolent sexist behaviours, specifically. SDO, but not RWA, and believing female gamers pose a threat to the collective image of gamers, predicted engagement in cyber VAWG, with SDO additionally predicting endorsement of cyber VAWG. Hostile (but not benevolent) sexism predicted endorsement of, but not engagement in cyber VAWG, consistent with the results of Study 2. Last, endorsement of cyber VAWG predicted engagement in cyber VAWG. In general, the DPM-inspired model in Study 3 provides evidence that social and group dominance threats and hostile sexism help explain the link between right-wing ideologies and cyber VAWG. 
In the ITT-inspired model, RWA and SDO predicted every threat type, with the exception of SDO not predicting intergroup anxiety. The lack of an intergroup anxiety-SDO relation contradicts previous literature (Bustillos \& Silván-ferrero, 2013; Matthews et al., 2009), and is inconsistent with Study 2. Consistent with literature (Sibley et al., 2007) and the differential effects hypothesis of the DPM (Duckitt \& Sibley, 2009), RWA predicted benevolent sexism and SDO predicted hostile sexism. However, RWA again also predicted hostile sexism. Unique to Study 3, less benevolent sexism predicted greater endorsement of cyber VAWG in the ITT-inspired, but not the DPM-inspired, model. This discrepancy is particularly notable, as the two variables have close to no relation to one another, suggesting that this finding may be a consequence of how the models are structured, and the large number of parameters that are included in them that are competing in explaining variance. In other words, this result may be a statistical artefact. Perceiving female gamers as threating to male gamers' values and success in controlling the gamer identity predicted hostile (but not benevolent) sexism. SDO, but not RWA, predicted group esteem threat, and benevolent and hostile sexism predicted endorsement of cyber VAWG. Last, SDO and endorsement of cyber VAWG predicted engagement in cyber VAWG. As only the relation between SDO, not RWA, and cyber VAWG outcomes was explained by perceived threats to the image of gamers posed by female gamers and hostile sexism, only partial support for Hypothesis 3 was found in the ITT-inspired model.

In summary, higher SDO emerged as the primary predictor of cyber VAWG outcomes across both models tested in Study 3. This was consistent with Study 2. Taken together, the DPM and ITT-inspired models provide partial evidence that greater sociopolitical ideologies (in particular, SDO) predict greater attitudes towards and engagement in cyber VAWG, as explained 
by people perceiving female as a threat (primarily social, group dominance, and group esteem threats) and holding hostile sexist attitudes. 


\section{General Discussion}

The recent and rapid growth of internet proliferation, accessibility, and platforms for communicating with others worldwide has facilitated the rise of a new breed of harmful behaviours that take advantage of these mature platforms and how integrated they are with our day-to-day lives. This emergence and proliferation of ways to target and harm others online necessitated a broader and more inclusive classification of harmful online behaviours, now referred to as cyber violence. However, understanding of these relatively newer behaviours is limited. A recent report by the United Nations presented a call-to-action for cyber violence against women and girls specifically, citing disproportionate victimization of online behaviours such as harassment and stalking (United Nations Entity for Gender Equality and the Empowerment of Women and United Nations Development Programme, 2015). Despite an abundance of psychological research on online gendered harassment (Barak, 2005; Barlett \& Coyne, 2014; Chisholm, 2006a; Henry \& Powell, 2015) and evidence that cybervictimization occurs disproportionately for women (Guo, 2016; Tokunaga, 2010), understanding of gendered cyber violence specifically is limited.

The present thesis sought to address this gap in the literature by evaluating the psychological properties of cyber VAWG and the roles of sociopolitical ideology, perceived threat, and sexism as antecedents of attitudes towards, and self-reported engagement in, cyber VAWG. These investigations were conducted on an undergraduate sample of male gamers (Study 1) and a diverse online sample of gamers (Studies 2 and 3). Participants for Studies 2 and 3 were mostly male, $87.3 \%$ and $90.7 \%$ respectively, and included representation from a highly diverse number of ethnicities from all over the world. It is worth noting again that results derived from Study 3 should be interpreted with caution as it is unclear how, and to what degree, 
participants were influenced by the livestream and archived video that made the survey link go 'viral.' Additional qualitative analyses are needed to explore how watching the live stream/video impacted participants. Such analyses, however, are beyond the scope of the present thesis.

\section{The Cyber VAWG Scale}

One of the key goals of the present thesis was to investigate the psychometric properties of a cyber VAWG measure. Across all three studies, factor analyses of the endorsement of cyber VAWG items revealed a single factor, suggesting that attitudes towards cyber VAWG represent a unidimensional psychological construct of general attitudes towards cyber VAWG, despite the specific behaviours and attitudes reflected in the items pulling from six different subdomains of cyber VAWG behaviour as defined by The Learning Network and United Nations' taxonomy of cyber VAWG behaviours (Baker et al., 2013; United Nations Entity for Gender Equality and the Empowerment of Women and United Nations Development Programme, 2015). The resulting scale, ranging from 28 to 36 items depending on the study, demonstrated excellent internal consistency $(\alpha$ 's $=.90-.93)$.

Items reflecting recruitment, harassment and spamming, and impersonation subdomains of cyber VAWG consistently loaded most strongly onto the single extracted factor in all three studies. More specifically, items assessing tricking using fake advertisements, creating fake profiles with the sole purpose of using them to harass, tricking into compromising situations, and making unprovoked sexual remarks were most closely and consistently associated with the cyber VAWG factor. Additionally, believing it is acceptable to share intimate photos of women online without their consent - classified under the malicious distribution category of cyber VAWG - had relatively strong loadings across all three studies. This is noteworthy, because distributing intimate media of women online is an almost prototypical example of cyber VAWG behaviour, 
and one that is even normalized to some degree with high traffic websites such as Reddit allowing users to freely share nude pictures of women. Conversely, the items that loaded relatively weaker, and the items that loaded weakest across studies did not show a clear pattern or adherence to one of the subdomains of cyber VAWG. In particular, items tapping bending the truth to get information out of women, using the information a woman puts out about herself onto the Internet to learn more about her, and believing that "Just because someone is telling a woman what they think, it does not mean it is threatening or intimidating" consistently loaded the weakest onto the single extracted factor, and were among those excluded from the scale score in Studies 1 and 3. Despite these patterns, items from within subdomains varied enough in the strength of their loadings that the unidimensional construct still best reflected general attitudes towards cyber VAWG.

Examination of the content of the weakly loading items suggests that they might be perceived as less harmful by participants. This could be due to poor face validity of the items, whereby participants confused the behaviours that the items intended to get at with more reasonable and frequently benign behaviours (e.g. using information about a woman to find other information about her; being expressive about opinions to women not being inherently threatening) or because those attitudes could be perceived as being well-meaning (e.g. bending the truth seeming subjectively harmless from the perceiver's end, but having the potential to cause harm in the future). As the three referenced items had some of the weakest factor loadings across all three studies, they should be dropped in future investigations that use this scale.

While many of the behaviours reflected in the cyber VAWG measure are transparent in their intent for harm (i.e. threatening real-life violence to a woman online), some are more ambiguous. For example, believing that it is okay to continually monitor the activities of a 
woman online could be contextualized as either stalking behaviour or concerned surveillance depending on who the target is. The target could be someone who the perpetrator is infatuated with, or a daughter of the perpetrator whom the perpetrator is concerned about. In both of these examples, there is potential for the target to feel that their security is being compromised; yet, the latter could be out of care and concern, whereas the former could reflect predatory behaviour. Thus, context and the relationship to the target affects the degree to which the behaviour is perceived as harmful and classifiable as an act of cyber violence. Although I recommended that the three items that are more ambiguous be dropped from the scale used in the present research, it would be fruitful for researchers to investigate attitudes towards behaviours that are contextdependent or those that can vary in their intent.

Despite cyber VAWG emerging as a unidimensional construct in the present thesis, it is worth investigating if cyber VAWG might also be conceptualized as two separate constructs that reflect different forms of the same negative attitudes to control for ambiguity in interpretation. This would parallel how Glick and Fiske (1996) distinguish hostile and benevolent sexism in their Ambivalent Sexism Theory. Items relating to the surveillance and tracking subdomain of cyber VAWG are likely candidates that can be perceived as benevolent in nature, as behaviours such as monitoring activity, tracing history, and tracking a woman via GPS or the information a woman has disclosed about herself online, have the potential to reflect the same protective paternalistic beliefs that underlie benevolent sexist attitudes (Glick \& Fiske, 1996) ${ }^{17}$. Testing models where each subdomain or smaller subsets of cyber VAWG items are modelled as distinct outcome variables could help identify potential relations between predictors and more specific

\footnotetext{
${ }^{17}$ Additional correlations were run on the relations between benevolent sexism and surveillance and tracking items of cyber VAWG individually, and as a subscale measure, but were not reported as they did not pertain to the three main hypotheses. No consistent, significant pattern of relations with benevolent sexism were found across samples.
} 
subdomains of cyber VAWG behaviours. This would also potentially elucidate whether there are a significant number of cyber VAWG behaviours that justify the existence of a benevolent cyber VAWG construct, or if it is merely tied to a handful of specific behaviours. Such an investigation would likely require additional scale items as a multi-dimensional construct was not supported in the present research.

\section{Hypothesis 1: Correlates of Cyber VAWG}

Across samples, higher RWA and SDO related to endorsement of cyber VAWG. Only SDO consistently related to greater engagement in cyber VAWG, as RWA did not relate to engagement in Study 1 - though the correlation approached significance $(p=.09)$. Thus, beliefs in social hierarchies and support of authoritarian social ideas related to endorsement of and engagement in cyber VAWG. RWA reflects an adherence to social conventions, social norms, and deference to authorities - with subversions of these beliefs justifying authoritarian aggression against those who dare to be deviant (Altemeyer, 1981). In online spaces such as gaming communities where men can perceive themselves as the resident authorities, the influence of female gamers could be perceived as threatening to and dissident from the social norms and conventions defined by male gamers, justifying cyber violence against them. Thus, for those higher in RWA, cyber VAWG might represent an appropriate response to those (i.e. female gamers) who violate social norms and conventions.

Comparatively, SDO, which consistently was either as-strong or stronger in relation to cyber VAWG outcomes relative to RWA, reflects support of inequalities in intergroup relations (Pratto et al., 1994). For those higher in SDO, therefore, cyber VAWG behaviours might provide effective means of preserving social hierarchies and keeping subordinate groups (i.e. female gamers) in their place. SDO might be more strongly related to cyber VAWG (vs. RWA) in the 
sample of gamers as the authority of male gamers may not be as well-defined and more presumed than enforced, whereas more basic views that the dominant group (i.e. male gamers) should be superior to the subordinate (i.e. female gamers) are not contingent upon specifics of jurisdictions of gamers. Cyber VAWG, then, joins a host of other negative attitudes that are related to higher RWA and SDO, such as negative attitudes towards international students (Charles-Toussaint \& Crowson, 2010), immigrants (Craig \& Richeson, 2014), ethnic minorities (Duckitt \& Sibley, 2007), ethnocentrism (Duckitt, 2004; Sibley, Robertson, \& Wilson, 2006), racism and anti-gay discrimination (for a meta-analysis, see Sibley et al., 2006), and generalized prejudice (Duckitt, 2004; Duckitt \& Sibley, 2007; Ekehammar Akrami, N., Gylje,M., Zakrisson, I., 2004).

Perceived threat related to endorsement of and engagement in cyber VAWG, as expected. Specifically, fears that female gamers are seeking to accrue power over male gamers (i.e. realistic threat), awkward to interact with (i.e. intergroup anxiety), and compromising existing gamer belief systems (i.e. symbolic threat) and social structures (i.e. social threat) related to support of cyber VAWG attitudes in some or all of the studies. Engagement in cyber VAWG behaviours related to all types of threat (except group dominance threat in Studies 2 and 3, and in Study 1 it only related to greater symbolic threat). The magnitude of the relations of realistic threat, symbolic threat, and intergroup anxiety with cyber VAWG outcome variables were generally moderate-to-strong, which is consistent with effect sizes reported on relations between these threats and other outgroup attitudes observed in the literature (for a meta-analysis, see Riek et al., 2006). It can be reasoned that the fear of female gamers usurping male gamers in power (i.e. realistic threat) and compromising traditional gamer values (i.e. symbolic threat) most consistently related to engagement in cyber VAWG. 
With respect to sexist attitudes, hostile sexism consistently related to endorsement of, and engagement in, cyber VAWG (except for a non-significant relation to engagement in cyber VAWG in Study 1), whereas benevolent sexism did not. Notably, benevolent sexism was the only predictor that was consistently unrelated to endorsement of and engagement in cyber VAWG, providing preliminary evidence that cyber VAWG generally does not relate to subjectively positive forms of gendered discrimination that define benevolent sexism, and the protective paternalistic beliefs that help comprise benevolent sexist attitudes that were speculated as being the reason for several poor factor loadings earlier (Glick \& Fiske, 1996).

Comparatively, hostile sexism, which related to cyber VAWG in all three studies, bears a greater conceptual similarity to cyber VAWG, echoing similar antipathic attitudes towards women that are transparent in their intent for harm. Last, endorsement of cyber VAWG was repeatedly related to engagement in cyber VAWG, showing a link between attitudes and behaviour.

\section{Hypothesis 2: Relations between Ideology with Threat and Sexist Attitudes}

In the DPM (Duckitt, 2001), RWA is proposed to associate uniquely with threats to social norms and structures, whereas SDO is proposed to associate uniquely with threats to group dominance. These threats directly reflect core values represented by each ideology, as people higher in RWA value social traditions and norms, and people higher in SDO believe in a rigid and impermeable hierarchical structure of social groups (Altemeyer, 1988; Sidanius \& Pratto, 1999). Yet, in the present research, RWA and SDO were correlated with both social threat and group dominance threat, except in Study 1, where only RWA related to group dominance threat. Notably however, in Studies 2 and 3, the magnitude of the SDO-group dominance threat relation was about twice that of the RWA-group dominance threat relation; and the RWA-social threat 
relation was somewhat stronger than the SDO-social threat relation, consistent with the DPM (Duckitt, 2001).

Currently, there is limited research on the relation between sociopolitical ideologies with ITT and group esteem threats (for exceptions, see: Bustillos \& Silván-ferrero, 2013; CharlesToussaint \& Crowson, 2010; Crowson, 2009; Rios, 2013; Ruffman et al., 2016; Uenal, 2016). Taking into account the restricted sample in Study 1 (and several non-significant findings between ideology and threat in Study 1), findings from the present thesis generally show that the robust association between right-wing ideologies and threat (Choma \& Hodson, 2017; Jost, Glaser, Kruglanski, \& Sulloway, 2003; Jost, Noorbaloochi, \& Van Bavel, 2014) extend to the threats identified in the Intergroup Threat Theory (Stephan \& Stephan, 2000). Of the research that has been conducted, both RWA and SDO have been shown to either relate or predict realistic (Charles-Toussaint \& Crowson, 2010; Ruffman et al., 2016; Uenal, 2016) and symbolic threat (Charles-Toussaint \& Crowson, 2010; Crowson, 2009; Rios, 2013; Ruffman et al., 2016; Uenal, 2016), and SDO has been shown to relate to intergroup anxiety (Bustillos \& Silvánferrero, 2013), consistent with the results of the present thesis. Hence, initial research does not seem to support distinct relations between ideology and particular types of ITT-threats.

One reason why sociopolitical ideologies did not distinctly relate to particular types of threat could be due to the presence of a latent factor reflecting global threat against female gamers. The four ITT threats, for instance, have been demonstrated to be moderately-to-strongly interrelated across the literature (Riek et al., 2006) and better predict greater prejudice against immigrants when combined into a latent threat factor (Stephan, Ybarra, Martnez, Schwarzwald, \& Tur-Kaspa, 1998). Similarly, it is possible that a latent threat factor of female gamers could exist in the cyber VAWG context. As noted in the Introduction, because many of the ITT types 
of threat can be conceptualized as both social and group dominance threats in the context of the DPM, it is possible that there is overlap in how the threats posed by female gamers are perceived. Specifically, a latent threat factor representing general threat of, or challenge to dominance by, female gamers could be more ecologically valid and have more explanatory value than assessing threats in isolation. A latent threat factor was not explored in the present thesis as the focus was on testing the DPM and ITT models; future research, however, on the relation between perceived threats and cyber VAWG should consider alternative models with a generalized threat factor.

With respect to right-wing ideology's relation to ambivalent sexist attitudes, Sibley and colleagues' (2007) meta-analysis found that RWA showed a moderate positive association with benevolent sexism, yet only a weak positive association with hostile sexism. In contrast, SDO showed a moderate positive correlation with hostile sexism, yet no relation to benevolent sexism. In the present thesis, RWA showed a moderate-to-strong relation with both benevolent and hostile sexism, whereas SDO showed a moderate-to-strong relation with hostile sexism, but a weak or non-significant relation with benevolent sexism. The relations between sociopolitical ideologies and ambivalent sexist attitudes were stronger in the present study across the board, compared to previous research (Sibley et al., 2007), a pattern that could be attributable to the sample or participants being primed by completing other measures (i.e. threat, cyber VAWG) that may have made negative gendered attitudes more salient or accessible. However, means for ideologies and ambivalent sexist attitudes were largely similar and within +/- 1 SD of what has been reported in the literature (see Sibley et al., 2007). It is worth noting that the size of the sample in Study 3 is several times larger than the cumulative number of observations included in 
Sibley and colleagues' (2007) meta-analysis. Thus, the present thesis provides additional evidence of a connection between right-wing ideology and sexist attitudes.

\section{Hypothesis 3: DPM-inspired and ITT-inspired Mediation Models}

In Studies 2 and 3, I tested whether sociopolitical ideologies, mediated by perceived threats and ambivalent sexist attitudes, predicted cyber VAWG. Specifically, DPM-inspired (i.e. social threat and group dominance threat; Duckitt, 2001) and ITT-inspired (i.e. realistic threat, symbolic threat, intergroup anxiety, and group esteem threat; Stephan \& Stephan, 2000) models were evaluated. Partial support was found for both models. In the DPM-inspired model, SDO consistently had an indirect effect on engagement in cyber VAWG, mediated by social threat, group dominance threat, and hostile sexism (SDO also had a direct effect on engagement in cyber VAWG). This mediated effect of the same mediators was observed for RWA in Study 2, but not in Study 3, indicating that gendered online aggression may be rooted more in support of hierarchical social structures (i.e. SDO) than in adherence to social norms and deference to authorities (i.e. RWA).

Both RWA and SDO predicted social threat and group dominance, inconsistent with the DPM (Duckitt, 2001). As noted earlier, this could reflect that a general (latent) threat of female gamers in the gaming community better captures the experience of threat than specific types of threat, or simply reflect the unique gender dynamics between male and female gamers. That is, unlike other intergroup contexts, men and women are more co-dependent on, and more frequently interact with one another across social contexts in their everyday social and familial lives, which can produce unique relations relative to other social boundaries between ingroups and outgroups (Glick \& Fiske, 1996). Furthermore, RWA consistently predicted hostile sexism, inconsistent with what is expected and found in the literature (Sibley et al., 2007). Notably, 
benevolent sexism consistently did not predict either cyber VAWG outcome variable; supplementing the notion that the sexist attitudes that underlie cyber VAWG are exclusively hostile in nature. Altogether, SDO, but not RWA, consistently emerged as a predictor of cyber VAWG outcomes in the DPM-inspired model, with social threat, group dominance threat, and hostile sexism serving as the primary mediators of those pathways.

The ITT-inspired model (Stephan \& Stephan, 2000) also provided partial support for Hypothesis 3 and showed many similarities to the DPM-inspired model overall, with RWA and SDO predicting all forms of threat included in the model (with the exception of RWA not predicting intergroup anxiety) and ambivalent sexist attitudes in the same pattern. Unlike the DPM-inspired model, SDO, but not RWA, consistently had an indirect effect on engagement of cyber VAWG. However, the indirect effects of RWA and SDO on endorsement of cyber VAWG were only observed in Study 2. Thus, as in the DPM-inspired model, SDO proved to be the more robust ideological predictor of cyber VAWG attitudes, mediated most consistently by group esteem threat and hostile sexism. With respect to sexism, benevolent sexism showed an unexpected, weak negative correlation with endorsement of cyber VAWG $(r=-.12)$. This is likely a statistical artefact that is a consequence of the high number of stages and parameters included in the models tested, as the relation between the two variables is virtually nonexistent ( $r$ $=.01$ ) and otherwise helps cement that cyber VAWG is primarily predicted by hostile, not benevolent, sexist attitudes. In the future, it is recommended that more concentrated models are used to better assess and control for overlaps in variance between variables and avoid such errant effects. For example, testing the mediating effect the DPM-threats between ideology and endorsement of cyber VAWG, without the inclusion of sexist attitudes or engagement in cyber VAWG. 
It is worth noting that the present thesis is novel in the use of ITT threats and group esteem threat as mediators between sociopolitical ideologies and negative attitudes and behaviour. To my knowledge, no other study has explored these threats as mediators between ideology and intergroup attitudes. Both RWA and SDO demonstrated weak-to-moderate predictive ability of all four types of threat included in the ITT-inspired model. Additionally, greater realistic threat reliably predicted hostile sexism, and greater group esteem threat reliably predicted endorsement of cyber VAWG. Based on the results from the ITT-inspired model it is posited that SDO, mediated by group esteem threat and hostile sexist attitudes, most reliably predicted cyber VAWG outcomes.

In summary, three consistent patterns across all of the tested models were observed. First, as expected, greater endorsement of cyber VAWG consistently predicts greater self-reported engagement in cyber VAWG. Second, greater hostile sexism predicted greater endorsement of cyber VAWG. Third, higher SDO consistently predicts both greater endorsement of and engagement in cyber VAWG; emerging as the preeminent predictor of cyber VAWG. People who fundamentally believe that some social groups (e.g. male gamers) are better than others (e.g. female gamers; i.e. SDO, Sidanius \& Pratto, 1999), are more likely to have positive attitudes towards cyber VAWG and engage more frequently in cyber VAWG behaviours. Comparatively, as both threats in the DPM-inspired model served as mediators, relative to only group esteem threat reliably predicting in the ITT-inspired model, it is believed that the DPM-inspired model was better at accounting for the predictive ability of sociopolitical ideologies on cyber VAWG outcomes. The three studies that comprise this thesis, while similar in format and psychological constructs measured, vary considerably from sample-to-sample. Study 1 is a small, homogenous sample of undergraduate male gamers, whereas the sample size in Study 3 dwarfs that of Studies 
1 and 2, but was likely influenced in uncertain ways by the content of the livestream that made the survey link go 'viral.' Hence, looking for consistent patterns across all three samples is difficult, and generalizations must be cautious.

\section{Practical Implications}

While psychological research on cyber VAWG is currently sparse and more basic research is required before reliable real-world applications of research can be made, understanding the psychological underpinnings of cyber VAWG from an intergroup perspective could help inform policy, the design of online communities, and interventions. As higher SDO, or support of group-based social hierarchies, and hostile sexism most reliably predicted cyber VAWG, online communication platforms can consider making hierarchy-enhancing and hostile sexist attitudes and behaviours against their terms of service and actively moderating against them. More broadly, Internet Service Providers (ISPs) and legislative bodies could adopt similar policies to help deter and implement grounds for enforcing consequences on those who exploit their access to the Internet for the sake of causing harm to others. In lieu of the finding that higher SDO predicts greater cyber VAWG, online communities that allow for users to fill out social group membership in their profiles or create and join groups of their own may want to consider removing or hiding design elements such as group rankings systems that could contribute to hierarchy-enhancing beliefs and greater intergroup conflict. Lastly, by understanding what specific attitudes or items from the cyber VAWG measure contribute most to endorsement of cyber VAWG, ISPs, NGOs, law enforcement, or stakeholders in online communities can establish digital markers of cyber VAWG and pre-empt cyber VAWG behaviours. On a smaller scale, moderators of smaller communities can identify response patterns in users and expunge them before they engage in conflicts that might push them to 
respond using act of cyber VAWG. On a larger scale, larger organizations can develop neural networks that trawl the Internet collecting information and converging evidence of perpetrators of cyber VAWG, similar to how organizations like Thorn: Digital Defends of Children collaborate with law enforcement agencies to track down child predators and traffickers. Additionally, possible interventions could target the specific behaviours perpetrators support in the endorsement of cyber VAWG scale, informing them of the consequences of those behaviours as a prejudice reduction method.

\section{Limitations and Future Directions}

It is important to note the limitations of the present thesis, in addition to those previously noted and discussed, and the directions that future research could take in order to supplement the results of the present thesis and expand understanding of cyber VAWG. Foremost, the present thesis only examined cyber VAWG through the narrow lens of the online gaming community and threats posed by female gamers specifically. Cyber VAWG attitudes are not specific or unique to gamers or the gaming community, and research in other social contexts and potential targets of cyber VAWG must be conducted in order to replicate findings, establish reliability and validity for the endorsement of cyber VAWG scale, and generalize the antecedents of cyber VAWG. Relatedly, the samples collected for this thesis were predominantly male (exclusively male in Study 1), and henceforth biased towards male interpretations and predictors of cyber VAWG. Similarly, many of the behaviours described and categorized by cyber VAWG are not exclusive to female targets, and thus future research into cyber violence against men and boys and whether cyber violence is gender-specific or a universal construct is necessary.

The nature of the samples in the present thesis presents an additional limitation. As previously noted, snowball recruiting methods were employed in Studies 2 and 3 when recruiting 
participants from online gaming communities. While these recruiting methods have the potential to attract a greater number of participants, publicly posting survey links onto the Internet in general relinquishes some control of the sample from the principal researcher, as the researcher can never be completely certain of how participants discovered and were introduced to the study. This is particularly pertinent to Study 3, which came into existence after the link for Study 2 went 'viral' via a YouTube account livestreaming the completion of the survey with added commentary. Because it is impossible to know the specifics of how viewing the livestream or archived video of the livestream primed or biased participants, we can only speculate on the effects and interpret the results of Study 3 with caution. Open-ended text responses collected alongside self-report scales will be analyzed in the future and could potentially provide insight into how participants in each study differed from one another. For example, analysing responses to what participants' think about the role of women in the gaming community might reveal different threats posed by female gamers or differing reasons and justifications for prejudice against them.

Last, the endorsement of cyber VAWG scale developed for the present thesis is considerably face-valid, and only represents a self-report index of cyber VAWG. Future investigations might consider employing experimental and observational methods to measure and evaluate cyber VAWG attitudes and behaviours. For example, researchers could create environmental conditions that may be more (vs. less) conducive to participants engaging in acts of cyber VAWG, such as leaving a friend of the participant's password for their social media in plain view and examining if they are more likely to use that information to breach their friend's security and tracking what specific behaviours they engage in once they have that access. 
Additionally, just as the cyber violence distinction evolved out of new technologies enabling new, harmful behaviours, the scale should be regularly updated to account for cyber VAWG behaviours that emerge as information technology systems evolve. Furthermore, as the behaviours that comprise cyber VAWG change, the relations with and predictive pathways to endorsement of and engagement in cyber VAWG could change and be retested. These include relations with the intergroup variables - as tested in the present thesis - as well as relations with other psychological constructs that have yet to be tested. For example, exploring how more contextual factors, such as feelings of being victimized by women can contribute to support of cyber VAWG and justify perpetrating cyber VAWG behaviours. The present investigation only examined the antecedents of cyber VAWG from an intergroup relations perspective, whereas the role of personality traits, psychopathology, empathy, aggression, and other potential predictors are yet to be explored, and should be a focus of research on cyber VAWG as reliable methods of evaluating cyber VAWG develop.

\section{Conclusion}

The present thesis developed and evaluated a scale measuring support of cyber VAWG. It was found that sociopolitical ideologies, perceived threats, and ambivalent sexist attitudes typical predictors of negative attitudes towards disadvantaged groups - related to and helped predict cyber VAWG outcomes. Across all three samples, greater hostile sexist attitudes predicted greater endorsement of cyber VAWG, and greater endorsement of cyber VAWG reliably predicted greater engagement in cyber VAWG. Most notably, higher SDO predicted both greater endorsement of and greater self-reported engagement in cyber VAWG. Thus, SDO emerged as the primary predictor of cyber VAWG. The present thesis represents an early investigation into the properties and antecedents of cyber VAWG, and future research should 
focus on validating the measure of endorsement of cyber VAWG and replicating the results of this thesis in non-gaming contexts, in addition to exploring predictors of cyber VAWG attitudes and behaviour outside of intergroup perspectives. 


\title{
Appendix A - Study 1 Consent Form
}

\author{
Ryerson University \\ Consent Agreement \\ Perceptions of Gamers
}

You are being asked to participate in a research study. Before consenting, it is important that you read the following information. You may ask as many questions as necessary to be sure that you understand what the study entails.

Investigators: Arvin Jagayat (Principal Investigator), Department of Psychology, Ryerson University, Toronto.

Dr. Becky Choma (Supervisor), Department of Psychology, Ryerson University, Toronto.

Purpose of the Study: The purpose of this social psychology study is to investigate gamer's beliefs about other gamers and society as a whole. 200 students at Ryerson enrolled in PSY 102/202 will be invited to participate in this research.

Description of the Study: If you decide to participate in the research, you will complete an online questionnaire. This study is expected to take approximately 30 minutes to complete. During the course of the study, you will be asked to do the following: Read a consent form and consent to participate (3 minutes), write thoughts you have about gamers and the perception of gamers (5 minutes), complete questionnaires about ideologies, emotions, and attitudes towards social groups (14 minutes), complete questionnaires about your demographics (3 minutes), and read a debriefing form (5 minutes).

What is Experimental in this Study: This study contains newly developed measures that are experimental in the sense that they have yet to demonstrate their usefulness, however the majority of the procedures or questionnaires have been used by other researchers and found to be useful. From a technical or procedural point of view, part of this study is considered "experimental", because by following the procedure described above, the study examines the impact of one variable (called the "independent variable") on another variable (called the "dependent variable"). More information about the independent and dependent variables will be provided at the end of the session.

Risks or Discomforts: This is a minimal risk study. However, some individuals may experience temporary boredom or mild emotional discomfort from answering questions on sensitive topics (e.g., beliefs about other social groups). Participants may choose to refuse to participate in any aspect of the research (e.g., responding to questionnaire items). Any discomfort is expected to be temporary and not greater than you might experience when in a typical day. If any aspect of this study makes you feel uncomfortable, you may temporarily or permanently discontinue your participation without penalty or loss of your course participation credit.

Benefits of the Study: There is no direct benefit to participants in this study although the information gained from the overall study may improve research on intergroup relations, and increase your knowledge of psychological research. When the session is over, we will describe the purpose and hypotheses of the study to you in more detail.

Confidentiality: You will not put your name or student number on the questionnaires. You will be asked to electronically consent (if you decide to participate) and your responses in this research will be anonymous and so there will be no way of linking your responses with your identity. 
The data from this study will be stored on password-protected computers and will be kept indefinitely to facilitate requests from other researchers to verify any research findings. Data will be deleted from the Qualtrics database 2 years after collection. If you choose to withdraw from the study your data will be destroyed/deleted. Consent forms will be kept for a minimum of 1 year.

Only your SONA ID will be included with your questionnaire responses so that we can identify your data should you wish to withdraw your data from the study. The online questionnaires are hosted by Qualtrics, a web survey company located in the USA and as such, is subject to U.S. laws; in particular, the US Patriot Act, which allows authorities access to the records of internet service providers. This survey or questionnaire does not ask for personal identifiers or any information that may be used to identify you. However, if you choose to participate in the survey, you understand that your responses to the survey questions will be stored, and can be accessed, in the USA. The security and privacy policy for the web survey company can be found at the following link: http://www.qualtrics.com/security-statement/'

Incentives to Participate: You will receive 0.5 participation credits to use towards your PSY 102/202 course at Ryerson. If you would prefer to 'walk through' the study (that is, if you would like to observe the research process but not provide any personal data), you will still be given the $0.5 \%$ credit assuming you have not already received the maximum allotted for research participation (currently $3 \%$ ).

Voluntary Nature of Participation: Participation in this study is voluntary. Your choice of whether or not to participate will not affect your grades or academic status. If you decide to participate, you are free to withdraw your consent and to stop your participation at any time without penalty or loss of benefits to which you are allowed. You can withdraw from the study by closing the browser. If you have answered questions prior to withdrawing, that data will not be saved or used in the findings of this study. Should you withdraw from the study, you will still be given your 0.5 credit.

Questions about the Study: If you have any questions about the research now, please ask. If you have questions later about the research, you may contact Arvin Jagayat or his supervisor, Dr. Becky Choma.

\section{Contact information}

Arvin Jagayat

Masters Student

Department of Psychology

Social and Political Psychology Lab

Email: arvin.jagayat@psych.ryerson.ca

Dr. Becky Choma

Assistant Professor

Department of Psychology

Social and Political Psychology Lab

Phone: 416-979-5000 ext. 3006

Email: becky.choma@psych.ryerson.ca

If you having any questions regarding your rights as a human subject and participant in this study, you may contact the Ryerson University Research Ethics Board for information: Ryerson Ethics Board, c/o Office of the Vice President, Research and Innovation, Ryerson University 350 Victoria Street Toronto, ON M5B 2K3, 416-979-5042 rebchair@ ryerson.ca

If completing any of these measurements raises concerns that you would like to discuss, please contact the: Centre for Student Development and Counselling (CSDC), Ryerson University Jorgenson Hall (JOR07C) 416-979-5195 csdc@ryerson.ca 


\section{If you any have questions about receiving your Psychology 102/202 credit for participation, please contact: thepool@psych.ryerson.ca}

Agreement: Your electronic consent below indicates that you have read the information in this agreement and have hand a chance to ask any questions you have about the study. Your signature also indicates that you agree to be in the study and have been told that you can change your mind any time during the study and withdraw from it. You have been given a copy of this agreement.

You have been told that by electronically consenting, you are not giving up any of your legal rights.

Please select the box indicating your choice:

$\square$ I CONSENT TO PARITICIPATE.

$\square$ I DO NOT CONSENT AND DO NOT WISH TO PARTICIPATE. 


\section{Appendix B - Study 1 Measures}

Below are copies of all of the measures administered in Study 1. Asterisks $(*)$ next to items indicate the most reliable items used in the computation of scores for the respective measures noted in the Methods.

\section{SONA ID}

Please enter your 6-digit SONA ID. Please note that this is NOT the same as your Ryerson ID. If you do not enter your 6-digit SONA ID, there will be no way for the researchers to give you credit for participation.

Group Identification (Luhtanen \& Crocker, 1992)

Please indicate how much you agree or disagree with each of the statements below, using the following scale:

$\begin{array}{rrrrrrrrr}\begin{array}{l}\text { Strongly } \\ \text { Disagree }\end{array} & 1 & 2 & 3 & 4 & 5 & 6 & 7 & \text { Strongly } \\ \text { Agree }\end{array}$

1. Overall, being a gamer has little to do with how I feel about myself.

2. Being a gamer is an important reflection of who I am.

3. Being a gamer is unimportant to my sense of what kind of a person I am.

4. In general, being a gamer is an important part of my self-image.

\section{General Gaming Questions}

How much would you consider yourself a gamer?

$\square$ Completely

$\square$ Somewhat

$\square$ A little

$\square$ Not at all

Please estimate how many hours a week you spend playing video games by completing the following sentence:

I spend hours and minutes playing video games every week. 
Please name your favourite, or one of your favourite video games:

\section{Role of Women in the Video Gaming Community}

We are interested in learning more about peoples' thoughts about women in the video gaming community. Please share any thoughts or feelings you may have about the role of women in the video gaming community:

\section{Dummy Questions}

As you may know, people can create bots to complete online surveys using random responses. Below are a few simple questions just to make sure you're not a computer. Please select the correct answers.

What color is the sky?
$\square$ Red
$\square$ Blue
$\square$ Yellow
$\square$ Green

How many hours are there in a day?
$\square 8$
$\square 16$
$\square 24$
$\square 32$

Social Dominance Orientation (Pratto et al., 1994; Ho et al. 2016)

Show how much you favour or oppose each idea below by selecting a number from 1 (STRONGLY OPPOSE) to 7 (STRONGLY FAVOUR) on the scale below. You can work quickly; your first feeling is generally best.

$\begin{array}{rrrrrrrrr}\begin{array}{c}\text { Strongly } \\ \text { Oppose }\end{array} & 1 & 2 & 3 & 4 & 5 & 6 & 7 & \text { Strongly } \\ & & & & & & & & \end{array}$

1. Some groups of people must be kept in their place.

2. It's probably a good thing that certain groups are at the top and other groups are at the bottom.

3. An ideal society requires some groups to be on top and others to be on the bottom.

4. Some groups of people are simply inferior to other groups.

5. Groups at the bottom are just as deserving as groups at the top.

6. No one group should dominate in society. 
7. Groups at the bottom should not have to stay in their place.

8. Group dominance is a poor principle.

9. We should not push for group equality.

10. We shouldn't try to guarantee that every group has the same quality of life.

11. It is unjust to try to make groups equal.

12. Group equality should not be our primary goal.

13. We should work to give all groups an equal chance to succeed.

14. We should do what we can to equalize conditions for different groups.

15. No matter how much effort it takes, we ought to strive to ensure that all groups have the same chance in life.

16. Group equality should be our ideal.

Right Wing Authoritarianism (Altemeyer, 1998)

Please circle your response, using the scale below.

1

2

3

Strongly Moderatel

Disagree $\mathbf{y}$

Disagree

\begin{abstract}
Slightly
Disagree
\end{abstract}

Neither

Disagree

Nor Agree
5

Slightly

Agree
6

7

Moderatel Strongly

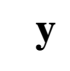

Agree

1. Gays and lesbians are just as healthy and moral as anybody else.

1

2

3

4

5

6

7

2. Atheists and others who have rebelled against the established religions are no doubt every bit as good and virtuous as those who attend church regularly.

1
2

3
4
5

6

7 
3. There are many radical, immoral people in our country today who are trying to ruin it for their godless purposes, whom the authorities should put out of action.
1
2
3
4
5
6
7

4. Our country will be destroyed someday if we do not smash the perversions eating away at our moral and traditional beliefs.
1
2
3
4
5
6
7

5. The situation in our country is getting so serious, the strongest methods would be justified if they eliminated the troublemakers and got us back to our true path.
1
2
3
4
5
6
7

6. Everyone should have their own lifestyle, religious beliefs, and sexual preferences, even if it makes them different from everyone else.
1
2
3
4
5
6
7

7. People should pay less attention to the Bible and the other old traditional forms of religious guidance, and instead develop their own personal standards of what is moral and immoral.
1
2
3
4
5
6
7

8. The only way our country can get through the crisis ahead is to get back to our traditional values, put some tough leaders in power, and silence the troublemakers spreading bad ideas.
1
2
3
4
5
6
7

9. There is nothing wrong with premarital sexual intercourse.

1

2

3

4

5

6

7

10. What our country really needs, instead of more "civil rights" is a good, stiff dose of law and order. 
11. Some of the best people in our country are those who are challenging our government, criticizing religion, and ignoring the "normal way" things are supposed to be done.
1
2
3
4
5
6
7

12. The facts on crime, sexual immorality, and the recent public disorders all show that we have to crack down harder on deviant groups and trouble-makers if we are going to save our moral standards and preserve law and order.

1
3
4
5
6

7

Realistic Threat (Stephan \& Stephan, 2000)

We are interested in your opinions about others that play video games. Please indicate the degree to which you agree or disagree with each of the statements below, using the following scale:

$\begin{array}{llllllllllll}\text { Not at All } & 1 & 2 & 3 & 4 & 5 & 6 & 7 & 8 & 9 & 10 & \text { Extremely }\end{array}$

1. Female gamers get more from the video gaming community than they contribute.*

2. More women playing video games and having a greater say in what makes someone a gamer or not is a good thing.

3. The rise in the number of female gamers has compromised the quality and type of games that game developers make.*

Symbolic Threat (Stephan \& Stephan, 2000)

We are interested in your opinions about others that play video games. Please indicate the degree to which you agree or disagree with each of the statements below, using the following scale:

$\begin{array}{llllllllllll}\text { Not at All } & 1 & 2 & 3 & 4 & 5 & 6 & 7 & 8 & 9 & 10 & \text { Extremely }\end{array}$

1. The presence of female gamers can serve to help improve depictions of women in games.

2. Female gamers' opinions about video games cause conflicts within video gaming communities.*

3. What female gamers value in their video games is problematic for long-time fans of video games.*

4. Because female gamers tend to like the video games they do, they are helping divert attention from games that deserve it more.*

5. New female gamers' views on video games can muzzle existing gamer's opinions.* 
Intergroup Anxiety (Stephan \& Stephan, 1985)

If you were in a group of female gamers, would you feel...

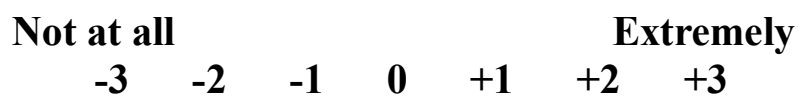
a) ... awkward
$\begin{array}{lllllll}-3 & -2 & -1 & 0 & +1 & +2 & +3\end{array}$
b) $\ldots$ self-conscious
$\begin{array}{lllllll}-3 & -2 & -1 & 0 & +1 & +2 & +3\end{array}$
c) ... happy
$\begin{array}{lllllll}-3 & -2 & -1 & 0 & +1 & +2 & +3\end{array}$
d) $\ldots$ accepted
$\begin{array}{lllllll}-3 & -2 & -1 & 0 & +1 & +2 & +3\end{array}$
e) $\ldots \underline{\text { confident }}$
$\begin{array}{lllllll}-3 & -2 & -1 & 0 & +1 & +2 & +3\end{array}$
f) $\ldots$ irritated
$\begin{array}{lllllll}-3 & -2 & -1 & 0 & +1 & +2 & +3\end{array}$
g) ... impatient
$\begin{array}{lllllll}-3 & -2 & -1 & 0 & +1 & +2 & +3\end{array}$
h) $\ldots$ defensive
$\begin{array}{lllllll}-3 & -2 & -1 & 0 & +1 & +2 & +3\end{array}$
i) ... suspicious
$\begin{array}{lllllll}-3 & -2 & -1 & 0 & +1 & +2 & +3\end{array}$
j) ... careful
$\begin{array}{lllllll}-3 & -2 & -1 & 0 & +1 & +2 & +3\end{array}$

Negative Stereotypes (Stephan et al., 1993)

Please estimate and select the percentage of female gamers who possess each of the following traits:

$\begin{array}{llllllllllll}\text { Talented } & 0 \% & 10 \% & 20 \% & 30 \% & 40 \% & 50 \% & 60 \% & 70 \% & 80 \% & 90 \% & 100 \% \\ \text { Competitive } & 0 \% & 10 \% & 20 \% & 30 \% & 40 \% & 50 \% & 60 \% & 70 \% & 80 \% & 90 \% & 100 \% \\ \text { Smart } & 0 \% & 10 \% & 20 \% & 30 \% & 40 \% & 50 \% & 60 \% & 70 \% & 80 \% & 90 \% & 100 \% \\ \text { Friendly } & 0 \% & 10 \% & 20 \% & 30 \% & 40 \% & 50 \% & 60 \% & 70 \% & 80 \% & 90 \% & 100 \% \\ \text { Competent } & 0 \% & 10 \% & 20 \% & 30 \% & 40 \% & 50 \% & 60 \% & 70 \% & 80 \% & 90 \% & 100 \% \\ \text { Attention-Seeking } & 0 \% & 10 \% & 20 \% & 30 \% & 40 \% & 50 \% & 60 \% & 70 \% & 80 \% & 90 \% & 100 \% \\ \text { Manipulative } & 0 \% & 10 \% & 20 \% & 30 \% & 40 \% & 50 \% & 60 \% & 70 \% & 80 \% & 90 \% & 100 \% \\ \text { Antisocial } & 0 \% & 10 \% & 20 \% & 30 \% & 40 \% & 50 \% & 60 \% & 70 \% & 80 \% & 90 \% & 100 \% \\ \text { Annoying } & 0 \% & 10 \% & 20 \% & 30 \% & 40 \% & 50 \% & 60 \% & 70 \% & 80 \% & 90 \% & 100 \%\end{array}$


Group Esteem Threat (Luhtanen \& Crocker, 1992)

We are all members of different social groups or social categories. Some of such social groups or categories pertain to gender, race, religion, nationality, ethnicity, and socioeconomic class. We would like you to consider your membership as a gamer, and respond to the following statements on the basis of how you feel about your gamer membership. There are no right or wrong answers to any of these statements; we are interested in your honest reactions and opinions. Please read each statement carefully, and respond by using the following scale:

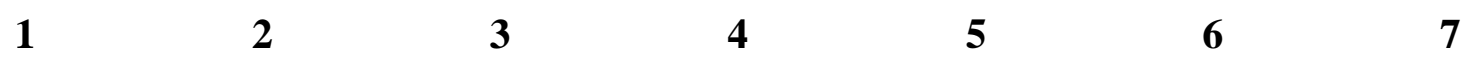

$\begin{array}{lcccccc}\text { Strongly } & \text { Disagree } & \begin{array}{c}\text { Disagree } \\ \text { Somewhat }\end{array} & \text { Neutral } & \begin{array}{c}\text { Agree } \\ \text { Somewhat }\end{array} & \text { Agree } & \text { Strongly } \\ \text { Disagree } & & & \text { Agree }\end{array}$

1. Female gamers compromise the public's perceptions of gamers.*

2. My willingness to identify as a gamer is diminished in the presence of female gamers.

3. I feel good about being a gamer, regardless of the number of female gamers there are.*

4. When more females identify as gamers, being a gamer becomes a less important part of my self-image.*

Group Dominance Threat (Charlesford \& Choma, 2013; Choma et al., 2016)

Please indicate the degree to which you agree or disagree with each of the statements below, using the following scale:

$\begin{array}{llllllll}1 & 2 & 3 & 4 & 5 & 6 & 7\end{array}$

$\begin{array}{ccccccc}\text { Strongly } & \text { Disagree } & \begin{array}{c}\text { Disagree } \\ \text { Somewhat }\end{array} & \text { Neutral } & \begin{array}{c}\text { Agree } \\ \text { Somewhat }\end{array} & \text { Agree } & \text { Strongly } \\ \text { Disagree } & & & & \text { Agree }\end{array}$

1. I hate when female gamers are portrayed as inferior to male gamers.*

2. I would like to see female gamers respected and protected by the gaming community and industry.*

3. I fear that the gaming community is not tolerant enough of female gamers.* 
4. The time and effort spent on protecting female gamers would be better spent on other issues.

Social Threat (Charlesford \& Choma, 2013; Choma et al., 2016)

Please indicate the degree to which you agree or disagree with each of the statements below, using the following scale:

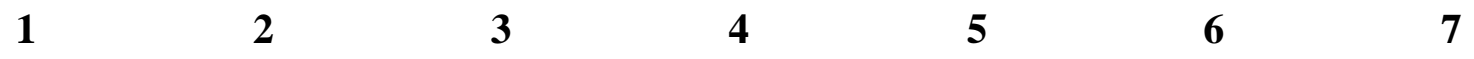

$\begin{array}{ccccccc}\text { Strongly } & \text { Disagree } & \begin{array}{c}\text { Disagree } \\ \text { Somewhat }\end{array} & \text { Neutral } & \begin{array}{c}\text { Agree } \\ \text { Somewhat }\end{array} & \text { Agree } & \text { Strongly } \\ \text { Disagree } & & & & \text { Agree }\end{array}$

1. Female gamers are a threat to gaming.*

2. It is clear that the welfare of the video gaming community is threatened by female gamers.*

3. Female gamers undermine traditional gamer values.*

4. Male gamers shouldn't be threatened by female gamers.

Ambivalent Sexism Inventory (Glick \& Fiske, 1996)

Below is a series of statements concerning men and women and their relationships in contemporary society. Please indicate the degree to which you agree or disagree with each statement using the following scale:

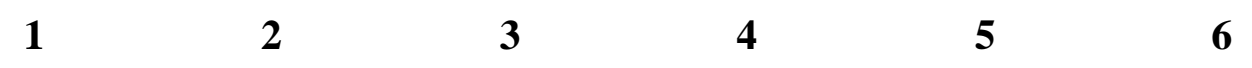

$\begin{array}{cccccc}\text { Disagree } & \text { Disagree } & \text { Disagree } & \text { Agree } & \text { Agree } & \text { Agree } \\ \text { Strongly } & \text { Somewhat } & \text { Slightly } & \text { Slightly } & \text { Somewhat } & \text { Strongly }\end{array}$

1. No matter how accomplished he is, a man is not truly complete as a person unless he has the love of a woman.

2. Many women are actually seeking special favors, such as hiring policies that favor them over men, under the guise of asking for "equality."

3. In a disaster, women ought not necessarily to be rescued before men.

4. Most women interpret innocent remarks or acts as being sexist.

5. Women are too easily offended. 
6. People are often truly happy in life without being romantically involved with a member of the other sex.

7. Feminists are not seeking for women to have more power than men.

8. Many women have a quality of purity that few men possess.

9. Women should be cherished and protected by men.

10. Most women fail to appreciate fully all that men do for them.

11. Women seek to gain power by getting control over men.

12. Every man ought to have a woman whom he adores.

13. Men are complete without women.

14. Women exaggerate problems they have at work.

15. Once a woman gets a man to commit to her, she usually tries to put him on a tight leash.

16. When women lose to men in a fair competition, they typically complain about being discriminated against.

17. A good woman should be set on a pedestal by her man.

18. There are actually very few women who get a kick out of teasing men by seeming sexually available and then refusing male advances.

19. Women, compared to men, tend to have a superior moral sensibility.

20. Men should be willing to sacrifice their own well being in order to provide financially for the women in their lives.

21. Feminists are making entirely reasonable demands of men.

22. Women, as compared to men, tend to have a more refined sense of culture and good taste.

\section{Endorsement of Cyber Violence Against Women and Girls}

For each of the following statements, please indicate the extent you agree or disagree using the scale below. Please remember that there are no right or wrong answers, and that your first responses are usually the most accurate.

$\begin{array}{llllllll}1 & 2 & 3 & 4 & 5 & 6 & 7\end{array}$

$\begin{array}{lcccccc}\text { Disagree } & \text { Disagree } & \text { Disagree } & \text { Neither } & \text { Agree } & \text { Agree } & \text { Agree } \\ \text { Strongly } & \text { Somewhat } & \text { Slightly } & \text { Agree or } & \text { Slightly } & \text { Somewhat } & \text { Strongly }\end{array}$


1. Obtaining a woman's personal information online without her permission is never okay.

2. If I want to access information about a woman online, I can do what I need to do to acquire it.

3. Even if I can, I am not free to bypass security to get information about a woman online.

4. It is okay to gain access to a live stream of a woman's webcam without her knowledge.

5. It is acceptable to gain unauthorized access to a woman's information on the Internet.

6. If I have figured out the password to a woman's account or profile, I can do what I want with it.

7. To get information about a woman, it's okay to pose as her online.

8. It is okay to impersonate someone online to learn more about a woman.

9. Pretending to be someone else when contacting a woman on the Internet is not okay.

10. It is not appropriate to forge identifying information of a woman on the Internet to learn more about her.

11. If a woman has an online presence, it is okay for me to use information she puts out there to learn more about her.

12. It is not okay to use the Internet to track down a woman.

13. There is nothing wrong with using the Internet to trace a woman's history.

14. It is unacceptable to use the Internet to trace where a woman has been.

15. Continually monitoring the activities of a woman online is totally fine.

16. It is not okay to use GPS or location tracking to find out where a woman is right now.

17. I have the right to express my anger towards a woman online as frequently as I wish.

18. Just because someone is telling a woman what they think, it does not mean it is threatening or intimidating.

19. There is potential harm in repeatedly messaging, calling or texting a woman.

20. It is fine to use the Internet to contact a woman even though she does not want to be contacted.

21. It is okay to use the Internet to tell a woman what I think about her, regardless of if she wants to know or not.

22. Spamming a woman's e-mail, social media, phone or voicemail with messages is wrong.

23. There is no problem with telling a woman to kill herself on the Internet.

24. It's okay to create new, anonymous online profiles or accounts for the sole purpose of using them to spam a woman with messages.

25. There's nothing wrong with threatening real life violence to a woman on the Internet.

26. There's nothing wrong with making unprovoked sexual remarks towards or sexual requests of a woman on the Internet.

27. It is okay to trick a woman into a compromising situation using the Internet.

28. There is nothing wrong with using fake advertisements or profiles to trick a woman online.

29. It is unacceptable to bend the truth on the Internet to get the information you want out of a woman.

30. It is okay to mislead a woman online. 
31. On the Internet, to convince a woman of something, sometimes you have to say things that are not entirely true.

32. It is okay to share intimate photos or videos of a woman online without her consent.

33. I do not have the right to spread fake stories or rumors about a woman online.

34. Twisting the truth about a woman online for my personal purposes is not right.

35. Threatening to release private information about a woman onto the Internet in order to get my way is acceptable thing to do.

36. I'm at liberty to send intimate photos of myself to any woman I choose.

37. Women have too much influence on the Internet today.

38. Women should know the risks of putting any information about themselves online.

39. With how pervasive Internet access is today, women should not be surprised if people use it to hunt down more information about them.

40. Women are fair and reasonable in the demands they express online.

41. Because of the lack of accountability and consequences online, it is acceptable to target and attack women online.

42. It is okay to express and perpetuate misogynistic beliefs online.

43. Greater anonymity on the Internet allows me to express my true feelings about women.

44. Attacking women online helps me gain followers, views, and/or popularity.

45. One of the advantages of attacking women online is that people acknowledge my opinions.

46. On the Internet, there is no issue with making sexual comments about women.

\section{Engagement in Cyber Violence Against Women and Girls}

We are interested in how often you participate in various online behaviours. Please indicate how often you engage in each behaviour using the following scale:

1

Never
2

Very
Rarely

3

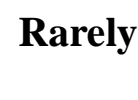

4

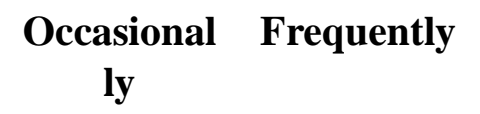

6

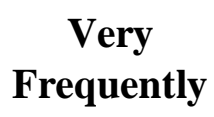

1. Gain access to a woman's online accounts or profiles.

2. Pretend to be someone I'm not when talking to a woman online.

3. Monitor a woman's activities online.

4. Spam or repeatedly expressing anger towards a woman online.

5. Intentionally mislead a woman online.

6. Post rumours or intimate photos of a woman online.

\section{Comments on Cyber VAWG in the Gaming Community}

In the past few years, the video gaming community has seen several news stories involving female members experiencing repeated harassment from many others on social media.

Have you heard of such events occurring recently in the video gaming community? 
$\square$ Yes

$\square$ No

We are interested in hearing any opinions or perspectives you may have on these news stories. Please share any thoughts or feelings you have about these news stories:

\section{Demographics}

1. Please indicate your gender by checking one of the boxes: Male Female Other

2. Please state your age:

3. Please indicate your ethnic background by checking all that apply.

$\square$ White/Caucasian

$\square$ Black

$\square$ South Asian

$\square$ Chinese

$\square$ Korean

$\square$ Japanese

$\square$ Southeast Asian

$\square$ Filipino

$\square$ Arab/West Asian

$\square$ Latin American

$\square$ Other, please specify:

4. Please indicate what country you currently live in:
$\square$ Brazil
$\square$ Canada
$\square$ China
$\square$ France
$\square$ Germany
$\square$ Japan
$\square$ Mexico 

$\square$ Russia
$\square$ South Korea
$\square$ Spain
$\square$ United Kingdom
$\square$ United States
$\square$ Other, please specify:

5. What is the highest level of education that you have completed?
$\square$ Less than high school graduate
$\square$ High school graduate
$\square$ Some college or university
$\square$ Completed college or university (Bachelor's Degree)
$\square$ Master's degree
$\square$ Doctoral degree

6. What is your best estimate of your household's total annual income from all sources, before taxes, in the past year?

$$
\begin{aligned}
& \square \text { Under } \$ 15,000 \\
& \square \$ 15,001-\$ 30,000 \\
& \square \$ 30,001-\$ 45,000 \\
& \square \$ 45,001-\$ 60,000 \\
& \square \$ 60,001-\$ 75,000 \\
& \square \$ 75,001-\$ 100,000 \\
& \square \$ 100,000-\$ 150,000 \\
& \square \text { Over } \$ 150,000
\end{aligned}
$$




\title{
Appendix C - Study 1 Debriefing Form
}

\author{
Project Title: The Roles of Ideology, Threat, and Sexist Attitudes in Cyber-Violence Against \\ Women and Girls \\ Principal Investigator: Arvin Jagayat \\ Supervisor: Dr. Becky Choma
}

Today, there are more women and girls playing video games than ever before (Internet Advertising Bureau UK, 2014). Recently, there have been several news stories about women in video gaming communities and companies being repeatedly attacked online. Victims of cyberbullying are more often women than they are men (Guo, 2016), and cyberbullies are more likely to be men than women. Despite how common gendered online harassment is, there has been little research into why people might engage in these types of behaviours. The goal of this research is to: (1) develop a scale that measures people's opinions about cyber violence against women and girls, and (2) explore whether certain beliefs, attitudes or perceptions that people hold relate to their opinions of cyber violence against women and girls.

The questions about people's opinions of cyber violence against women and girls was developed to measure the 6 categories of behaviour that make up cyber violence against women and girls, according to a report by the United Nations in 2015. These 6 categories are hacking, impersonation, surveillance and tracking, harassment and spamming, recruitment, and malicious distribution.

In addition to these questions, participants also completed measures of ideology, specifically rightwing authoritarianism (RWA) and social dominance orientation (SDO). People higher in RWA strongly support maintaining existing social norms, strictly obey legitimate authorities (like the police), and endorse harsh authoritarian aggression (meaning people higher in RWA believe it is OK to harshly punish people who violate social norms). People higher in SDO believe that some social groups are better than others. People higher in RWA and SDO tend to hold negative attitudes toward groups such as immigrants, gays and lesbians, the homeless, and women. With respect to their attitudes toward women, people higher in RWA tend to endorse benevolent sexism which is a condescending type of sexism that promotes traditional gender roles. People higher in SDO often endorse hostile sexism which is an overtly hateful and belittling type of sexism (Glick \& Fiske, 1996; Sibley et al., 2007). This connection between beliefs (RWA, SDO) and sexist attitudes is partially because people may feel that other groups (e.g. female gamers) are troublesome to interact with, or have too much influence on their group identity, culture, or social norms (Duckitt \& Sibley, 2009; Riek et al., 2006). It should be noted that your participation in this study does not necessarily mean that you are high in RWA, SDO, or sexist attitudes, and you will neither be provided your scores on those measures or individually labelled as being high or low on any of them.

In this study, it is expected that strongly subscribing to RWA and SDO, holding sexist attitudes, and believing that female gamers are difficult to interact with and have too much influence, will lead to more favourable attitudes toward cyber behaviours such as hacking, impersonation, surveillance and tracking, harassment and spamming, recruitment and malicious distribution.

We believe that this research program is very important to the field of social psychology. We thank you so much for being a part of it.

Because anonymity is very important to this study, we ask that you please DO NOT DISCUSS any part of this study with your friends, peers, or classmates who are likely to take part in the study until after DECEMBER 17, 2016. If you wish to discuss the study with people who have 
already participated in the study, or people who never will participate (e.g., parents, friends who do not attend Ryerson), that is acceptable.

The study will be compromised if you discuss its procedures with potential participants. In psychological research, it is often very important that participants are unaware of the specific procedures and hypotheses of a study before they participate in it, otherwise participants may not respond in a natural way (either to be helpful or because they believe the researcher wishes them to respond in a specific way). We hope you have learned something about psychological research processes by taking part in this study. Please print or save this debriefing form for your own records.

We hope you will learn something about intergroup relations from participating in this research. For further reading on the topics studied by this research, please see the bottom of this page.

Thank you for your time and support in participating in this study! If you have any questions or concerns, please feel free to contact any of the researchers (see above).

After reading this debriefing form, you may choose to opt out of your responses being used in this study by selecting the box below:

I choose to withdraw my responses from use in this study.

If you having any questions regarding your rights as a human subject and participant in this study, you may contact the Ryerson University Research Ethics Board for information: Ryerson Ethics Board, c/o Office of the Vice President, Research and Innovation, Ryerson University, 350 Victoria Street Toronto, ON M5B 2K3, 416-979-5042 rebchair@ryerson.ca

\title{
If you have question about receiving your Psychology 102/202 credit for participation please contact the researcher first, and then: thepool@psych.ryerson.ca
}

\author{
Arvin Jagayat \\ Masters Student \\ Department of Psychology \\ Social and Political Psychology Lab \\ Email: arvin.jagayat@psych.ryerson.ca \\ Dr. Becky Choma \\ Assistant Professor \\ Department of Psychology \\ Social and Political Psychology Lab \\ Phone: 416-979-5000 ext. 3006 \\ Email: becky.choma@psych.ryerson.ca
}




\section{References for further reading:}

Duckitt, J., \& Sibley, C. G. (2009). A Dual-Process Motivational Model of Ideology, Politics, and Prejudice. Psychological Inquiry, 20(2-3), 98-109. http://doi.org/10.1080/10478400903028540

Shaw, A. (2012). Do you identify as a gamer? gender, race, sexuality, and gamer identity. New Media \& Society, 14(1), 28-44. doi:http://dx.doi.org/10.1177/1461444811410394

United Nations Entity for Gender Equality and the Empowerment of Women and United Nations Development Programme. (2015). Cyber Violence Against Women and Girls. Retrieved from http://www.unwomen.org/en/digital-library/publications/2015/9/cyber-violence-against-women-and-girls 


\section{Appendix D - Factor Loadings Table for the first PAF conducted in Study 1}

\begin{tabular}{|c|c|c|c|c|c|c|c|c|c|}
\hline \multirow[b]{2}{*}{ Item } & \multicolumn{9}{|c|}{ Factor } \\
\hline & 1 & 2 & 3 & 4 & 5 & 6 & 7 & 8 & 9 \\
\hline $\begin{array}{l}\text { is okay to trick a wom } \\
\text { mpromising situation } \\
\text { ternet. (RC) }\end{array}$ & .976 & .029 & -.027 & .004 & .015 & .040 & -.038 & .002 & -.034 \\
\hline $\begin{array}{l}\text { here's nothing wrong with making } \\
\text { nprovoked sexual remarks towards or } \\
\text { xual requests of a woman on the } \\
\text { ternet. (HS) }\end{array}$ & .967 & .008 & -.089 & .080 & .080 & .042 & -.040 & -.029 & .042 \\
\hline $\begin{array}{l}\text { te } \\
\text { onto the } \\
\text { vay is }\end{array}$ & .963 & -.006 & -.076 & -.015 & -.080 & .022. & -.005 & -.014 & .004 \\
\hline $\begin{array}{l}\text { e intimate photos or } \\
\text { an online without her }\end{array}$ & .940 & .031 & -.082 & -.031 & .171 & .092 & .023 & .013 & -.021 \\
\hline $\begin{array}{l}\text { rew, anonymous } \\
\text { accounts for the sole } \\
\text { them to spam a }\end{array}$ & .777 & -.093 & .152 & .100 & .294 & -.020 & .006 & .137 & -.092 \\
\hline $\begin{array}{l}\text { using } \\
\text { s to trick }\end{array}$ & .775 & .391 & .053 & .000 & -.202 & -.093 & -.052 & -.002 & .042 \\
\hline $\begin{array}{l}\text { vith } \\
\text { ence to a }\end{array}$ & .718 & .061 & -.065 & -.116 & .503 & .113 & .123 & .005 & -.047 \\
\hline oman, it's & -.084 & .897 & .035 & -.144 & .411 & -.028 & -.021 & .074 & .020 \\
\hline er & .258 & .838 & 80 & -.047 & -.071 & .214 & -.283 & -.084 & -.03 \\
\hline ontin & -.123 & .759 & -.127 & .266 & .014 & .050 & .173 & -.284 & .093 \\
\hline $\begin{array}{l}\text { nt or profile, I can do } \\
h \text { it. (IM) }\end{array}$ & .170 & .735 & .203 & .154 & -.057 & -.207 & -.088 & .090 & .073 \\
\hline nline to & -.032 & .720 & -.041 & .161 & .500 & -.068 & .028 & .004 & 135 \\
\hline $\begin{array}{l}\text { is okay to mislead a woman online. } \\
\text { (C) }\end{array}$ & .394 & .652 & .120 & -.184 & -.049 & -.104 & .068 & -.037 & .000 \\
\hline $\begin{array}{l}\text { is acceptable to gain unauthorized } \\
\text { ceess to a woman's information on } \\
\text { e Internet. (HC) }\end{array}$ & . 185. & .566 & .020 & -.069 & .097 & -.120 & -.002 & -.009 & .242 \\
\hline
\end{tabular}


If I want to access information about a

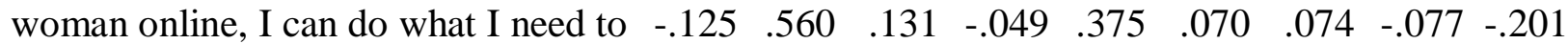
do to acquire it. (HC)

I do not have the right to spread fake

$\begin{array}{lllllllllllll}\text { stories or rumors about a woman } & & -032 & -.034 & .097 & .000 & -.094 & -.106 & .044 & -.003 & -.034\end{array}$ online. (MD)

Twisting the truth about a woman

$\begin{array}{llllllllllll}\text { online for my personal purposes is not } & -.050 & -.026 & .820 & -.156 & .007 & -.079 & .012 & -.001 & .005\end{array}$

right. (MD)

Even if I can, I am not free to bypass

$\begin{array}{lllllllllll}\text { security to get information about a } & & -.007 & .127 & .762 & .084 & -.100 & .084 & -.180 & -.072 & -.013\end{array}$ woman online. (HC)

Spamming a woman's e-mail, social

$\begin{array}{lllllllllllllll}\text { media, phone or voicemail with } & & & -.065 & -.054 & .517 & .017 & .070 & .464 & -.058 & -.179 & .014\end{array}$

messages is wrong. (HS)

It is unacceptable to bend the truth on

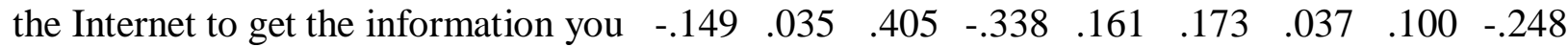

want out of a woman. (RC)

It is fine to use the Internet to contact a

$\begin{array}{llllllllll}\text { woman even though she does not want } & -.051 & .087 & .026 & .061 & .113 & .119 & .111 & -.294 & .064\end{array}$ to be contacted. (HS)

It is okay to use the Internet to tell a woman what I think about her, regardless of if she wants to know or $\begin{array}{lllllllll}.208 & -.210 & -.017 & .691 & -.078 & -.082 & .345 & .020 & -.029\end{array}$ not. (HS)

If a woman has an online presence, it is okay for me to use information she puts out there to learn more about her. $\begin{array}{llllllllll}-.220 & .100 & -.273 & .499 & -.025 & -.029 & -.053 & .154 & -.252\end{array}$ (ST)

On the Internet, to convince a woman of something, sometimes you have to say things that are not entirely true.

$\begin{array}{lllllllll}.229 & .057 & .135 & .479 & .112 & -.243 & .052 & .099 & -.084\end{array}$ (RC)

There is no problem with telling a $\begin{array}{llllllllll}\text { woman to kill herself on the Internet. } & .327 & .023 & .003 & .278 & .691 & .051 & -.114 & .195 & -.059\end{array}$ (HS)

It is okay to gain access to a live $\begin{array}{llllllllllll}\text { stream of a woman's webcam without } & .105 & .223 & -.074 & -.089 & .594 & .056 & -.088 & .034 & -.148\end{array}$ her knowledge. (HC)

It is not appropriate to forge $\begin{array}{lllllllllll}\text { identifying information of a woman on } & -.024 & .041 & -.116 & -.088 & .139 & .842 & -.124 & .07 & .251\end{array}$ the Internet to learn more about her. (IM)

There is potential harm in repeatedly

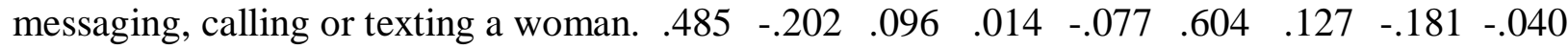
(HS) 
It is unacceptable to use the Internet to trace where a woman has been. (ST)

$\begin{array}{lllllllll}.018 & .104 & .153 & .277 & -.104 & .315 & -.144 & .236 & -.078\end{array}$

I have the right to express my anger

$\begin{array}{llllllllll}\text { towards a woman online as frequently } & -.113 & .268 & -.064 & .183 & -.017 & .022 & .608 & -.086 & .0254\end{array}$ as I wish. (HS)

Just because someone is telling a woman what they think, it does not mean it is threatening or intimidating. $\begin{array}{lllllllll}.001 & -. .330 & -.023 & .162 & -.087 & -.113 & .518 & .139 & .058\end{array}$ (HS)

I'm at liberty to send intimate photos of myself to any woman I choose.

$\begin{array}{lllllllll}.277 & .290 & -.018 & -.071 & -.236 & -.046 & .0486 & .032 & -.185\end{array}$ (MD)

It is not okay to use GPS or location $\begin{array}{lllllllllll}\text { tracking to find out where a woman is } & .048 & -.179 & -.068 & -.118 & .162 & -.016 & .035 & .894 & .125\end{array}$ right now. (ST)

It is not okay to use the Internet to track down a woman. (ST) $\begin{array}{lllllllll}-.025 & .072 & .096 & .025 & -.114 & .353 & .216 & .491 & .458\end{array}$ Pretending to be someone else when $\begin{array}{llllllllll}\text { contacting a woman on the Internet is } & -.081 & .131 & .003 & -.068 & -.185 & .226 & .093 & .183 & .855\end{array}$ not okay. (IM)

There is nothing wrong with using the $\begin{array}{llllllllll}\text { Internet to trace a woman's history. } & -1248 & .318 & -.030 & .245 & .032 & .076 & .237 & .202 & -.352\end{array}$ (ST)

Note (HC) = Hacking; $(\mathrm{IM})=$ Impersonation; $(\mathrm{ST})=$ Surveillance \& Tracking; $(\mathrm{HS})=$ Harassment \& Spamming; $(\mathrm{RC})=$ Recruitment; $(\mathrm{MD})=$ Malicious Distribution. 


\section{Appendix E - Sample Online Recruitment Post}

Post Title: Hey $<$ community name $>$, I'm doing some research on gamers and I need your help!

Post content: Hi <community name>!

Video games and gaming communities have changed a lot over the past decade. In that time, the perceptions society has of gamers, and even the perceptions gamers have about other gamers have changed. I'm conducting a study to look at this in more detail as a part of my Master's thesis, and I would appreciate any and all gamers that can help out by completing a short survey on the matter. This survey is open to people who play video games in any capacity (i.e. whether you play video games frequently or infrequently, on computers, gaming consoles or smartphones), as I am interested in hearing a variety of perspectives from a variety of different kinds of gamers. You also need to be at least 18 years of age to participate. If you complete the survey, you can put yourself into a draw to win one of five $\$ 25 \mathrm{CAD}$ gift cards (or the closest equivalent in your home country) to your digital gaming store of choice (Steam, XBL, PSN, eShop).

It will only take 15 minutes of your time, every response is anonymous, and I would encourage anyone who is interested to both read the consent form carefully and respond as honestly as possible to all of the questions. If you are interested, you can complete the survey at the following link:

<survey link here>

Thanks for helping out! After you've completed the survey, feel free to share the link with any other gamers you may know!

2017 is shaping up to be another big year in gaming. If you win one of the gift cards in the draw, what game(s) will you put your store credit towards? 


\section{Appendix F - Contact Message sent to Community Leaders}

Dear <community/contact name>,

My name is Arvin, and I am both a lifelong gamer and currently a Master's student at Ryerson University conducting research for my thesis. As a gamer, I have noticed that the role of women in gaming has become a prominent point of discussion in the gaming community in the past few years. Recent metrics have pointed towards a rise in the proportion of female gamers, however in that same time many gamers and gaming communities have become familiar with several highly publicized incidents of online aggression towards female community members. In light of this, there seems to be a growing public sentiment that all gamers hold extreme and negative attitudes about women. These claims are unwarranted. As a part of my research, I am interested in studying, empirically, the opinions about the role of women in gaming that our community does hold, and how their beliefs and emotions might influence them. I believe that this research will provide a more representative and fairer assessment of the beliefs held by those in gaming communities, more generally. I am contacting you today to ask for permission to post a survey link to your community in order to help gather data for my Master's thesis.

The survey will take around 15 minutes to complete. It will be completely anonymous, as I will not collect any identifying information nor report the specific names of the online communities I recruit from. Additionally, participants will have the opportunity to enter a survey-wide draw for one of five gift cards to their choice of digital gaming store (i.e. Steam, eShop, PSN, XBL). For the draw, participants will provide their e-mail contact information in a separate survey.

I can understand any hesitation for participation because I empathize with concerns that survey results can be twisted to mislabel communities, and hence want to make it clear that the goal of my research is NOT to misrepresent gamers and the gaming community as a whole. I wish to give the gaming community an opportunity to share their thoughts, ideas, and opinions on this important issue. The beliefs I am looking at in my research are NOT specific to gamers; something that will be made very clear should this research ever be published. Some individuals may hold more negative views, while many might hold positive views.

Your community and several other gaming communities across the Internet have been contacted to participate because of their relative size and activity of members. Additionally, if you are interested, I would be happy to post a report of the general findings to your community once they are available. I hope that, with your permission, you will help me collect the data I need to conduct my research and help contribute to collective understanding of the more general beliefs I am investigating.

If you have any questions at all, please let me know. Thank you for your time and I eagerly look forward to hearing from you.

Kind Regards,

- Arvin 


\title{
Appendix G - Studies 2 \& 3 Consent Form
}

\author{
Ryerson University \\ Consent Agreement \\ Perceptions of Gamers
}

You are being asked to participate in a research study. Before consenting, it is important that you read the following information. You may ask as many questions as necessary to be sure that you understand what the study entails.

Investigators: Social Psychology Lab, Department of Psychology, Ryerson University, Toronto.

Purpose of the Study: The purpose of this study is to investigate gamer's beliefs about other gamers and society as a whole. 400 members of online video gaming communities will be invited to participate in this research.

Description of the Study: If you decide to participate in the research, you will complete an online questionnaire. This study is expected to take approximately 15 minutes to complete. During the course of the study, you will be asked to do the following: Read a consent form and consent to participate (3 minutes), write thoughts you have about gamers and the perception of gamers ( 2 minutes), complete questionnaires about ideologies, emotions, and attitudes towards social groups (5 minutes), complete questionnaires about your demographics (2 minutes), and read a debriefing form (3 minutes).

Risks or Discomforts: This is a minimal risk study. However, some individuals may experience temporary boredom or mild emotional discomfort from answering questions on sensitive topics (e.g., beliefs about other social groups). Participants may choose to refuse to participate in any aspect of the research (e.g., responding to questionnaire items). Any discomfort is expected to be temporary and not greater than you might experience when in a typical day. If any aspect of this study makes you feel uncomfortable, you may temporarily or permanently discontinue your participation without penalty at any given time.

Benefits of the Study: There is no direct benefit to participants in this study although the information gained from the overall study may improve research on intergroup relations, and increase your knowledge of psychological research. When the session is over, we will describe the purpose and hypotheses of the study to you in more detail.

Confidentiality: Your name will not be associated with our responses. Your responses in this research will be anonymous and so there will be no way of linking your responses with your identity. Similarly, the contact information provided in the separate survey for entry into the draw for store credit, while linked from the main survey, cannot be connected back to individual responses in the main survey. This information will be kept confidential until the study is complete, at which point the winners will be drawn, store credits will be awarded, and the draw survey data will be destroyed.

The data from this study will be stored on password-protected computers and will be kept indefinitely to facilitate requests from other researchers to verify any research findings. Data will be deleted from the Qualtrics database 2 years after collection. If you choose to withdraw from the study your data will be destroyed/deleted. Consent forms will be kept for a minimum of 1 year.

This survey uses Qualtrics, which is a United States of American (USA) company. Consequently, Qualtrics or USA authorities may access survey data in some forms (e.g., aggregate usage information) and under strict policies. Qualtrics employs a variety of security features to make sure that the data collected are not 
accessible by outside bodies. More information on Qualtrics' security systems can be viewed here: https://www.qualtrics.com/security-statement/. Information regarding their protective privacy policy is available here: https://www.qualtrics.com/privacy-statement/. Although Qualtrics usually stores IP address data, we have deactivated that function for this study.

Incentives to Participate: Upon completion of the study, participants will be eligible to enter into a draw for one of five gift cards of \$25 CAD (or the closest equivalent in their region) in store credit for the digital video gaming store of their choice (one of: Steam, Xbox Games Store, Playstation Store, or Nintendo eShop). If acquiring a gift card is not possible in a winner's region, a money order in the value of the store credit will be sent. At the end of the study, participants will be linked to a separate survey (that is not connectable to their responses in the main survey) for entry into the draw, in which they will provide a contact e-mail, their country, and preferred video gaming store to receive credit for. The probability of winning the draw for each participant will be 5 out of the total number of participants for this study. Winners will be notified via the contact e-mail they provide in the draw survey when data collection for this study is complete.

Voluntary Nature of Participation: Participation in this study is voluntary. If you decide to participate, you are free to withdraw your consent and to stop your participation at any time without penalty or loss of benefits to which you are allowed. You can withdraw from the study by closing the browser. If you have answered questions prior to withdrawing, that data will not be saved or used in the findings of this study.

Questions about the Study: If you have any questions about the research now or later about the research, you may contact the Social Psychology Lab:

\section{Contact information}

\section{Social Psychology Lab}

Department of Psychology

Ryerson University

Toronto, Ontario

Email: gamerstudy@psych.ryerson.ca

If you having any questions regarding your rights as a human subject and participant in this study, you may contact the Ryerson University Research Ethics Board for information: Ryerson Ethics Board, c/o Office of the Vice President, Research and Innovation, Ryerson University 350 Victoria Street Toronto, ON M5B 2K3, 416-979-5042 rebchair@ryerson.ca

If completing any of these measurements raises concerns that you would like to discuss, please contact the: Centre for Student Development and Counselling (CSDC), Ryerson University Jorgenson Hall (JOR07C) 416-979-5195 csdc@ ryerson.ca

Agreement: Your electronic consent below indicates that you have read the information in this agreement and have hand a chance to ask any questions you have about the study. Your signature also indicates that you agree to be in the study and have been told that you can change your mind any time during the study and withdraw from it. You may print screen or copy this agreement for your own records.

You have been told that by electronically consenting, you are not giving up any of your legal rights.

Please select the box indicating your choice: 
$\square$ I CONSENT TO PARITICIPATE.

$\square$ I DO NOT CONSENT AND DO NOT WISH TO PARTICIPATE. 


\section{Appendix H - Studies 2 \& 3 Measures}

Below are copies of all of the measures administered in Studies 2 and 3. Superscripted numbers next to items indicate the items included in the computation of scores for the respective study number.

Group Identification (Luhtanen \& Crocker, 1992)

Please indicate how much you agree or disagree with each of the statements below, using the following scale:

$\begin{array}{ccccccccc}\begin{array}{c}\text { Strongly } \\ \text { Disagree }\end{array} & 1 & 2 & 3 & 4 & 5 & 6 & 7 & \begin{array}{c}\text { Strongly } \\ \text { Agree }\end{array}\end{array}$

1. Being a gamer is an important reflection of who I am.

\section{General Gaming Questions}

Please estimate how many hours a week you spend playing video games by completing the following sentence:

I spend hours and minutes playing video games every week.

Please name your favourite, or one of your favourite video games:

\section{Role of Women in the Video Gaming Community}

We are interested in learning more about peoples' thoughts about women in the video gaming community. Please share any thoughts or feelings you may have about the role of women in the video gaming community:

\section{Dummy Questions}

As you may know, people can create bots to complete online surveys using random responses. Below are a few simple questions just to make sure you're not a computer. Please select the correct answers.

What color is the sky?
$\square \operatorname{Red}$
$\square$ Blue
$\square$ Yellow
$\square$ Green 
How many hours are there in a day?
$\square 8$
$\square 16$
$\square 24$
$\square 32$

Social Dominance Orientation (Pratto et al., 1994; Ho et al. 2016)

Show how much you favour or oppose each idea below by selecting a number from 1 (STRONGLY OPPOSE) to 7 (STRONGLY FAVOUR) on the scale below. You can work quickly; your first feeling is generally best.

$\begin{array}{rrrrrrrrr}\begin{array}{c}\text { Strongly } \\ \text { Oppose }\end{array} & 1 & 2 & 3 & 4 & 5 & 6 & 7 & \text { Strongly } \\ & & & & & & & & \end{array}$

1. An ideal society requires some groups to be on top and others to be on the bottom.

2. Some groups of people are simply inferior to other groups.

3. No one group should dominate in society.

4. Groups at the bottom are just as deserving as groups at the top.

5. Group equality should not be our primary goal.

6. It is unjust to try to make groups equal.

7. We should do what we can to equalize conditions for different groups.

8. We should work to give all groups an equal chance to succeed.

Right Wing Authoritarianism (Altemeyer, 1998)

Please circle your response, using the scale below.

1

2

3

Strongly Moderatel

Disagree y Disagree

Disagree
4

Neither

Disagree

Nor Agree
5

6

7

$\begin{array}{ccc}\text { Slightly } & \text { Moderatel } & \text { Strongly } \\ \text { Agree } & \mathbf{y} & \text { Agree } \\ & \text { Agree } & \end{array}$

1. Gays and lesbians are just as healthy and moral as anybody else.
1
2
3
4
5
6
7

2. Atheists and others who have rebelled against the established religions are no doubt every bit as good and virtuous as those who attend church regularly.

1

2
3
5

6

7 
3. There are many radical, immoral people in our country today who are trying to ruin it for their godless purposes, whom the authorities should put out of action.
1
2
3
4
5
6
7

4. Our country will be destroyed someday if we do not smash the perversions eating away at our moral and traditional beliefs.
1
2
3
4
5
6
7

5. The situation in our country is getting so serious, the strongest methods would be justified if they eliminated the troublemakers and got us back to our true path.
1
2
3
4
5
6
7

6. Everyone should have their own lifestyle, religious beliefs, and sexual preferences, even if it makes them different from everyone else.
1
2
3
4
5
6
7

7. People should pay less attention to the Bible and the other old traditional forms of religious guidance, and instead develop their own personal standards of what is moral and immoral.
1
2
3
4
5
6
7

8. The only way our country can get through the crisis ahead is to get back to our traditional values, put some tough leaders in power, and silence the troublemakers spreading bad ideas.
1
2
3
4
5
6
7

9. There is nothing wrong with premarital sexual intercourse.
1
2
3
4
5
6
7

10. What our country really needs, instead of more "civil rights" is a good, stiff dose of law and order. 
11. Some of the best people in our country are those who are challenging our government, criticizing religion, and ignoring the "normal way" things are supposed to be done.

12. The facts on crime, sexual immorality, and the recent public disorders all show that we have to crack down harder on deviant groups and trouble-makers if we are going to save our moral standards and preserve law and order.
1
2
3
4
5
6
7

Realistic Threat (Stephan \& Stephan, 2000)

We are interested in your opinions about others that play video games. Please indicate the degree to which you agree or disagree with each of the statements below, using the following scale:

$\begin{array}{llllllllllll}\text { Not at All } & 1 & 2 & 3 & 4 & 5 & 6 & 7 & 8 & 9 & 10 & \text { Extremely }\end{array}$

1. Female gamers get more from the video gaming community than they contribute.

2. The rise in the number of female gamers has compromised the quality and type of games that game developers make.

Symbolic Threat (Stephan \& Stephan, 2000)

We are interested in your opinions about others that play video games. Please indicate the degree to which you agree or disagree with each of the statements below, using the following scale:

$\begin{array}{llllllllllll}\text { Not at All } & 1 & 2 & 3 & 4 & 5 & 6 & 7 & 8 & 9 & 10 & \text { Extremely }\end{array}$

1. What female gamers value in their video games is problematic for long-time fans of video games.

2. Female gamers' views on video games can muzzle existing gamer's opinions. 
Intergroup Anxiety (Stephan \& Stephan, 1985)

If you were in a group of female gamers, would you feel...

\begin{tabular}{ccccccc} 
Not at all & & & \multicolumn{3}{c}{ Extremely } \\
$\mathbf{- 3}$ & $\mathbf{- 2}$ & $\mathbf{- 1}$ & $\mathbf{0}$ & $\mathbf{+ 1}$ & $\mathbf{+ 2}$ & $\mathbf{+ 3}$ \\
-3 & -2 & -1 & 0 & +1 & +2 & +3 \\
-3 & -2 & -1 & 0 & +1 & +2 & +3 \\
-3 & -2 & -1 & 0 & +1 & +2 & +3 \\
-3 & -2 & -1 & 0 & +1 & +2 & +3 \\
-3 & -2 & -1 & 0 & +1 & +2 & +3 \\
-3 & -2 & -1 & 0 & +1 & +2 & +3
\end{tabular}

Negative Stereotypes (Stephan et al., 1993)

Please estimate and select the percentage of female gamers who possess each of the following traits:

$\begin{array}{llllllllllll}\text { Talented } & 0 \% & 10 \% & 20 \% & 30 \% & 40 \% & 50 \% & 60 \% & 70 \% & 80 \% & 90 \% & 100 \%\end{array}$

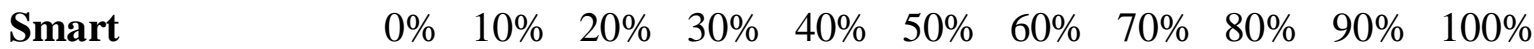

$\begin{array}{llllllllllll}\text { Friendly } & 0 \% & 10 \% & 20 \% & 30 \% & 40 \% & 50 \% & 60 \% & 70 \% & 80 \% & 90 \% & 100 \%\end{array}$

$\begin{array}{llllllllllll}\text { Attention-Seeking } & 0 \% & 10 \% & 20 \% & 30 \% & 40 \% & 50 \% & 60 \% & 70 \% & 80 \% & 90 \% & 100 \%\end{array}$

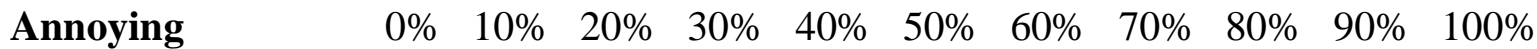

$\begin{array}{llllllllllll}\text { Unattractive } & 0 \% & 10 \% & 20 \% & 30 \% & 40 \% & 50 \% & 60 \% & 70 \% & 80 \% & 90 \% & 100 \%\end{array}$

Please rate how favorable each of the following traits are on a scale from 1 (VERY

FAVORABLE) to 10 (VERY FAVORABLE):

$\begin{array}{llllllllllll}\text { Not at All } & 1 & 2 & 3 & 4 & 5 & 6 & 7 & 8 & 9 & 10 & \text { Extremely }\end{array}$

1. Talented

2. Smart

3. Friendly

4. Attention-Seeking

5. Annoying

6. Unattractive 
Group Esteem Threat (Luhtanen \& Crocker, 1992)

We are all members of different social groups or social categories. Some of such social groups or categories pertain to gender, race, religion, nationality, ethnicity, and socioeconomic class. We would like you to consider your membership as a gamer, and respond to the following statements on the basis of how you feel about your gamer membership. There are no right or wrong answers to any of these statements; we are interested in your honest reactions and opinions. Please read each statement carefully, and respond by using the following scale:

1

Strongly

Disagree 2

3

Disagree

Disagree Somewhat
4

Neutral
5

Agree
6

7

Agree Strongly

Agree

1. Female gamers compromise the public's perceptions of gamers. ${ }^{2}$

2. My willingness to identify as a gamer is diminished in the presence of female gamers. $^{23}$

3. I feel good about being a gamer, regardless of the number of female gamers there are. $^{3}$

Group Dominance Threat (Charlesford \& Choma, 2013; Choma et al., 2016)

Please indicate the degree to which you agree or disagree with each of the statements below, using the following scale:

1

Strongly

Disagree
2

Disagree

Disagree

Somewhat
4

5

6

7
Agree Somewhat
Agree

Strongly

Agree

1. I would like to see female gamers respected and protected by the gaming community and industry.

2. I fear that the gaming community is not tolerant enough of female gamers.

Social Threat (Charlesford \& Choma, 2013; Choma et al., 2016)

Please indicate the degree to which you agree or disagree with each of the statements below, using the following scale:

1

Strongly
2

3

Disagree
Disagree Somewhat
4

Neutral

Agree Somewhat
7

Agree

Strongly 
1. It is clear that the welfare of the video gaming community is threatened by female gamers.

2. Female gamers undermine traditional gamer values.

Ambivalent Sexism Inventory (Glick \& Fiske, 1996; Rollero, Glick \& Stefano, 2014)

Below is a series of statements concerning men and women and their relationships in contemporary society. Please indicate the degree to which you agree or disagree with each statement using the following scale:

\section{1} 2 3

Disagree Disagree Disagree Agree
5

Agree
6

Agree

\section{Strongly Somewhat Slightly Slightly Somewhat Strongly \\ Strongly Somewhat Slightly Slightly Somewhat Strongly}

1. Many women have a quality of purity that few men possess.

2. Women should be cherished and protected by men.

3. Women seek to gain power by getting control over men.

4. Every man ought to have a woman whom he adores.

5. Men are incomplete without women.

6. Women exaggerate problems they have at work.

7. Once a woman gets a man to commit to her, she usually tries to put him on a tight leash.

8. When women lose to men in a fair competition, they typically complain about being discriminated against.

9. Many women get a kick out of teasing men by seeming sexually available and then refusing male advances.

10. Women, compared to men, tend to have a superior moral sensibility.

11. Men should be willing to sacrifice their own well being in order to provide financially for the women in their lives.

12. Feminists are making unreasonable demands of men. 


\section{Endorsement of Cyber Violence Against Women and Girls}

For each of the following statements, please indicate the extent you agree or disagree using the scale below. Please remember that there are no right or wrong answers, and that your first responses are usually the most accurate.

1

Disagree

Strongly
2

Disagree
3

Disagree Slightly
4

Neither Agree or Disagree
5

Agree

Slightly
6

Agree

Somewhat Strongly

1. Obtaining a woman's personal information online without her permission is never okay.

2. If I want to access information about a woman online, I can do what I need to do to acquire it.

3. Even if I can, I am not free to bypass security to get information about a woman online.

4. It is okay to gain access to a live stream of a woman's webcam without her knowledge.

5. It is acceptable to gain unauthorized access to a woman's information on the Internet.

6. If I have figured out the password to a woman's account or profile, I can do what I want with it.

7. To get information about a woman, it's okay to pose as her online.

8. It is okay to impersonate someone online to learn more about a woman.

9. Pretending to be someone else when contacting a woman on the Internet is not okay.

10. It is not appropriate to forge identifying information of a woman on the Internet to learn more about her.

11. If a woman has an online presence, it is okay for me to use information she puts out there to learn more about her.

12. It is not okay to use the Internet to track down a woman.

13. There is nothing wrong with using the Internet to trace a woman's history.

14. It is unacceptable to use the Internet to trace where a woman has been.

15. Continually monitoring the activities of a woman online is totally fine.

16. It is not okay to use GPS or location tracking to find out where a woman is right now.

17. I have the right to express my anger towards a woman online as frequently as I wish.

18. Just because someone is telling a woman what they think, it does not mean it is threatening or intimidating.

19. There is potential harm in repeatedly messaging, calling or texting a woman.

20. It is fine to use the Internet to contact a woman even though she does not want to be contacted.

21. It is okay to use the Internet to tell a woman what I think about her, regardless of if she wants to know or not.

22. Spamming a woman's e-mail, social media, phone or voicemail with messages is wrong.

23. There is no problem with telling a woman to kill herself on the Internet.

24. It's okay to create new, anonymous online profiles or accounts for the sole purpose of using them to spam a woman with messages. 
25. There's nothing wrong with threatening real life violence to a woman on the Internet.

26. There's nothing wrong with making unprovoked sexual remarks towards or sexual requests of a woman on the Internet.

27. It is okay to trick a woman into a compromising situation using the Internet.

28. There is nothing wrong with using fake advertisements or profiles to trick a woman online.

29. It is unacceptable to bend the truth on the Internet to get the information you want out of a woman.

30. It is okay to mislead a woman online.

31. On the Internet, to convince a woman of something, sometimes you have to say things that are not entirely true.

32. It is okay to share intimate photos or videos of a woman online without her consent.

33. I do not have the right to spread fake stories or rumors about a woman online.

34. Twisting the truth about a woman online for my personal purposes is not right.

35. Threatening to release private information about a woman onto the Internet in order to get my way is acceptable thing to do.

36. I'm at liberty to send intimate photos of myself to any woman I choose.

37. Women have too much influence on the Internet today.

38. Women should know the risks of putting any information about themselves online.

39. With how pervasive Internet access is today, women should not be surprised if people use it to hunt down more information about them.

40. Women are fair and reasonable in the demands they express online.

41. Because of the lack of accountability and consequences online, it is acceptable to target and attack women online.

42. It is okay to express and perpetuate misogynistic beliefs online.

43. Greater anonymity on the Internet allows me to express my true feelings about women.

44. Attacking women online helps me gain followers, views, and/or popularity.

45. One of the advantages of attacking women online is that people acknowledge my opinions.

46. On the Internet, there is no issue with making sexual comments about women.

\section{Engagement in Cyber Violence Against Women and Girls}

We are interested in how often you participate in various online behaviours. Please indicate how often you engage in each behaviour using the following scale:

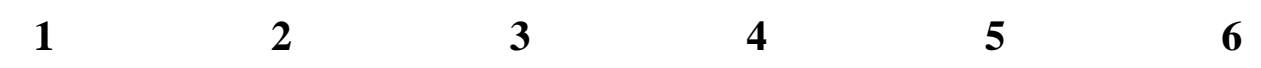

$\begin{array}{cccccc}\text { Never } & \begin{array}{c}\text { Very } \\ \text { Rarely }\end{array} & \text { Rarely } & \begin{array}{c}\text { Occasional } \\ \text { ly }\end{array} & \text { Frequently } & \begin{array}{c}\text { Very } \\ \text { Frequently }\end{array}\end{array}$

1. Gain access to a woman's online accounts or profiles.

2. Pretend to be someone I'm not when talking to a woman online.

3. Monitor a woman's activities online. 
4. Spam or repeatedly expressing anger towards a woman online.

5. Intentionally mislead a woman online.

6. Post rumours or intimate photos of a woman online.

\section{Comments on Cyber VAWG in the Gaming Community}

In the past few years, the video gaming community has seen several news stories involving female members experiencing repeated harassment from many others on social media.

Have you heard of such events occurring recently in the video gaming community?

$$
\begin{aligned}
& \square \text { Yes } \\
& \square \text { No }
\end{aligned}
$$

We are interested in hearing any opinions or perspectives you may have on these news stories. Please share any thoughts or feelings you have about these news stories:

\section{Demographics}

1. Please indicate your gender by checking one of the boxes: Male Female Other

2. Please state your age:

3. Please indicate your ethnic background by checking all that apply.

$\square$ White/Caucasian

$\square$ Black

$\square$ South Asian

$\square$ Chinese

$\square$ Korean

$\square$ Japanese

$\square$ Southeast Asian

$\square$ Filipino

$\square$ Arab/West Asian

$\square$ Latin American

$\square$ Other, please specify:

4. Please indicate what country you currently live in:

$\square$ Brazil 


$\square$ Canada
$\square$ China
$\square$ France
$\square$ Germany
$\square$ Japan
$\square$ Mexico
$\square$ Russia
$\square$ South Korea
$\square$ Spain
$\square$ United Kingdom
$\square$ United States
$\square$ Other, please specify:

5. What is the highest level of education that you have completed?
$\square$ Less than high school graduate
$\square$ High school graduate
$\square$ Some college or university
$\square$ Completed college or university (Bachelor's Degree)
$\square$ Master's degree
$\square$ Doctoral degree

6. What is your best estimate of your household's total annual income from all sources, before taxes, in the past year, in your country's currency?

$$
\begin{aligned}
& \square \text { Under } \$ 15,000 \\
& \square \$ 15,001-\$ 30,000 \\
& \square \$ 30,001-\$ 45,000 \\
& \square \$ 45,001-\$ 60,000 \\
& \square \$ 60,001-\$ 75,000
\end{aligned}
$$




\section{$\square \$ 75,001-\$ 100,000$ \\ $\square \$ 100,000-\$ 150,000$ \\ $\square$ Over $\$ 150,000$}

7. How did you first find out about this study? Please select all that apply. (Study 2 only)

$\square$ Forum or message board

$\square$ Facebook

$\square$ Twitter

$\square$ E-mail

$\square$ Text

$\square$ From a friend

$\square$ Other social media

$\square$ From an article or blog post

$\square$ Other, please specify 


\title{
Appendix I - Studies 2 \& 3 Debriefing Form
}

\author{
Project Title: The Roles of Ideology, Threat, and Sexist Attitudes in Cyber-Violence Against \\ Women and Girls \\ Principal Investigator: Social Psychology Lab
}

Today, there are more women and girls playing video games than ever before (Internet Advertising Bureau UK, 2014). Recently, there have been several news stories about women in video gaming communities and companies being repeatedly attacked online. Victims of cyberbullying are more often women than they are men (Guo, 2016), and cyberbullies are more likely to be men than women. Despite how common gendered online harassment is, there has been little research into why people might engage in these types of behaviours. The goal of this research is to: (1) develop a scale that measures people's opinions about cyber violence against women and girls, and (2) explore whether certain beliefs, attitudes or perceptions that people hold relate to their opinions of cyber violence against women and girls.

The questions about people's opinions of cyber violence against women and girls was developed to measure the 6 categories of behaviour that make up cyber violence against women and girls, according to a report by the United Nations in 2015. These 6 categories are hacking, impersonation, surveillance and tracking, harassment and spamming, recruitment, and malicious distribution.

In addition to these questions, participants also completed measures of ideology, specifically rightwing authoritarianism (RWA) and social dominance orientation (SDO). People higher in RWA strongly support maintaining existing social norms, strictly obey legitimate authorities (like the police), and endorse harsh authoritarian aggression (meaning people higher in RWA believe it is OK to harshly punish people who violate social norms). People higher in SDO believe that some social groups are better than others. People higher in RWA and SDO tend to hold negative attitudes toward groups such as immigrants, gays and lesbians, the homeless, and women. With respect to their attitudes toward women, people higher in RWA tend to endorse benevolent sexism which is a condescending type of sexism that promotes traditional gender roles. People higher in SDO often endorse hostile sexism which is an overtly hateful and belittling type of sexism (Glick \& Fiske, 1996; Sibley et al., 2007). This connection between beliefs (RWA, SDO) and sexist attitudes is partially because people may feel that other groups (e.g. female gamers) are troublesome to interact with, or have too much influence on their group identity, culture, or social norms (Duckitt \& Sibley, 2009; Riek et al., 2006). It should be noted that your participation in this study does not necessarily mean that you are high in RWA, SDO, or sexist attitudes, and you will neither be provided your scores on those measures or individually labelled as being high or low on any of them.

In this study, it is expected that strongly subscribing to RWA and SDO, holding sexist attitudes, and believing that female gamers are difficult to interact with and have too much influence, will lead to more favourable attitudes toward cyber behaviours such as hacking, impersonation, surveillance and tracking, harassment and spamming, recruitment and malicious distribution.

We believe that this research program is very important to the field of social psychology. We thank you so much for being a part of it.

Because anonymity is very important to this study, we ask that you please DO NOT DISCUSS any part of this study with anyone who is likely to take part in the study until after APRIL 29, 2017. If you wish to discuss the study with people who have already participated in the study, or people who never will participate (e.g., non-gamers), that is acceptable. Similarly, you are 
encouraged to share the link to this study with other gamers you may know as long as you do not discuss any parts of the study with them beforehand.

The study will be compromised if you discuss its procedures with potential participants. In psychological research, it is often very important that participants are unaware of the specific procedures and hypotheses of a study before they participate in it, otherwise participants may not respond in a natural way (either to be helpful or because they believe the researcher wishes them to respond in a specific way). We hope you have learned something about psychological research processes by taking part in this study. Please print or save this debriefing form for your own records.

We hope you will learn something about intergroup relations from participating in this research. For further reading on the topics studied by this research, please see the bottom of this page.

Thank you for your time and support in participating in this study! If you have any questions or concerns, please feel free to contact the researchers (see below).

After reading this debriefing form, you may choose to opt out of your responses being used in this study by selecting the box below:

I choose to withdraw my responses from use in this study.

If you having any questions regarding your rights as a human subject and participant in this study, you may contact the Ryerson University Research Ethics Board for information: Ryerson Ethics Board, c/o Office of the Vice President, Research and Innovation, Ryerson University, 350 Victoria Street Toronto, ON M5B 2K3, 416-979-5042 rebchair@ryerson.ca

Social Psychology Lab

Department of Psychology

Ryerson University

Toronto, Ontario

Email: gamerstudy@psych.ryerson.ca

References for further reading:

Duckitt, J., \& Sibley, C. G. (2009). A Dual-Process Motivational Model of Ideology, Politics, and Prejudice. Psychological Inquiry, 20(2-3), 98-109. http://doi.org/10.1080/10478400903028540

Shaw, A. (2012). Do you identify as a gamer? gender, race, sexuality, and gamer identity. New Media \& Society, 14(1), 28-44. doi:http://dx.doi.org/10.1177/1461444811410394

United Nations Entity for Gender Equality and the Empowerment of Women and United Nations Development Programme. (2015). Cyber Violence Against Women and Girls. Retrieved from http://www.unwomen.org/en/digital-library/publications/2015/9/cyber-violence-against-women-and-girls 


\section{Appendix J - Studies 2 \& 3 Incentive Draw Survey}

Thank you for taking part in the study. Below are a few questions to help enter you into the draw for store credit prizes.

1. Please provide a valid e-mail address, which will be used to contact you if you win one of the prizes:

2. Please provide your country (this is the region that the store credit will be bought for):

3. Please indicate the digital store you would prefer to receive credit for:

$\square$ Nintendo eShop

$\square$ Playstation Store

$\square$ Steam Store

$\square$ Xbox Games Store 


\section{Appendix K - Factor Loadings Table for the first PAF conducted in Study 2}

\begin{tabular}{|c|c|c|c|c|c|c|c|}
\hline \multirow[b]{2}{*}{ Item } & \multicolumn{7}{|c|}{ Factor } \\
\hline & 1 & 2 & 3 & 4 & 5 & 6 & 7 \\
\hline $\begin{array}{l}\text { It is okay to trick a woman into a } \\
\text { compromising situation using the Internet. } \\
\text { (RC) }\end{array}$ & .797 & .128 & -.158 & -.210 & -.098 & -.154 & -.038 \\
\hline $\begin{array}{l}\text { It's okay to create new, anonymous online } \\
\text { profiles or accounts for the sole purpose of } \\
\text { using them to spam a woman with } \\
\text { messages. (HS) }\end{array}$ & .756 & .178 & -.181 & -.187 & -.091 & -.116 & -.214 \\
\hline $\begin{array}{l}\text { There is nothing wrong with using fake } \\
\text { advertisements or profiles to trick a } \\
\text { woman online. (RC) }\end{array}$ & .751 & .171 & -.184 & -.181 & -.085 & -.096 & .014 \\
\hline $\begin{array}{l}\text { There's nothing wrong with making } \\
\text { unprovoked sexual remarks towards or } \\
\text { sexual requests of a woman on the } \\
\text { Internet. (HS) }\end{array}$ & .738 & -.227 & -.208 & -.103 & .035 & -.043 & .028 \\
\hline It is okay to mislead a woman online. (RC) & .694 & -.018 & -.016 & -.120 & -.024 & -.232 & .226 \\
\hline $\begin{array}{l}\text { It is fine to use the Internet to contact a } \\
\text { woman even though she does not want to } \\
\text { be contacted. (HS) }\end{array}$ & .691 & -.083 & -.083 & -.097 & .086 & .176 & -.065 \\
\hline $\begin{array}{l}\text { It is okay to use the Internet to tell a } \\
\text { woman what I think about her, regardless } \\
\text { of if she wants to know or not. (HS) }\end{array}$ & .660 & -.477 & .014 & -.059 & .089 & .134 & -.146 \\
\hline $\begin{array}{l}\text { To get information about a woman, it's } \\
\text { okay to pose as her online. (IM) }\end{array}$ & .660 & .441 & .215 & -.104 & .086 & -.094 & -.075 \\
\hline $\begin{array}{l}\text { There is no problem with telling a woman } \\
\text { to kill herself on the Internet. (HS) }\end{array}$ & .653 & -.168 & -.276 & -.135 & -.068 & .134 & .361 \\
\hline $\begin{array}{l}\text { Continually monitoring the activities of a } \\
\text { woman online is totally fine. (ST) }\end{array}$ & .645 & -.115 & .184 & -.027 & -.325 & -.058 & -.117 \\
\hline $\begin{array}{l}\text { If I have figured out the password to a } \\
\text { woman's account or profile, I can do what } \\
\text { I want with it. (IM) }\end{array}$ & .641 & .278 & .263 & -.134 & .156 & -.012 & .038 \\
\hline $\begin{array}{l}\text { It is okay to share intimate photos or } \\
\text { videos of a woman online without her } \\
\text { consent. (MD) }\end{array}$ & .633 & .347 & .270 & -.112 & .170 & .159 & -.018 \\
\hline $\begin{array}{l}\text { I do not have the right to spread fake } \\
\text { stories or rumors about a woman online. } \\
\text { (MD) }\end{array}$ & .633 & -.025 & -.235 & .202 & -.003 & -.131 & .055 \\
\hline $\begin{array}{l}\text { There is potential harm in repeatedly } \\
\text { messaging, calling or texting a woman. } \\
\text { (HS) }\end{array}$ & .632 & -.145 & -.173 & .009 & -.031 & .160 & -.125 \\
\hline I have the right to express my anger & .605 & -.475 & -.047 & -.039 & .176 & .049 & -.081 \\
\hline
\end{tabular}

towards a woman online as frequently as I wish. (HS) 
On the Internet, to convince a woman of something, sometimes you have to say things that are not entirely true. (RC)

It is okay to impersonate someone online to learn more about a woman. (IM)

I'm at liberty to send intimate photos of myself to any woman I choose. (MD)

$\begin{array}{lllllllll}\text { Obtaining a woman's personal information } & .575 & -.078 & .184 & .090 & .009 & -.044 & .317\end{array}$ online without her permission is never okay. (HC)

There is nothing wrong with using the Internet to trace a woman's history. (ST) Spamming a woman's e-mail, social media, phone or voicemail with messages is wrong. (HS)

If a woman has an online presence, it is okay for me to use information she puts out there to learn more about her. (ST) There's nothing wrong with threatening real life violence to a woman on the Internet. (HS)

It is not okay to use the Internet to track down a woman. (ST)

If I want to access information about a woman online, I can do what I need to do to acquire it. (HC)

It is okay to gain access to a live stream of a woman's webcam without her knowledge. (HC)

Twisting the truth about a woman online for my personal purposes is not right.

(MD)

It is not okay to use GPS or location

tracking to find out where a woman is right now. (ST)

Threatening to release private information about a woman onto the Internet in order to get my way is acceptable thing to do.

(MD)

It is acceptable to gain unauthorized access to a woman's information on the Internet. (HC)

Pretending to be someone else when contacting a woman on the Internet is not okay. (IM)

\begin{tabular}{|c|c|c|c|c|c|c|}
\hline .601 & -.146 & -.080 & -.064 & .022 & -.210 & -.034 \\
\hline .585 & .298 & .211 & -.017 & .072 & -.224 & -.115 \\
\hline .579 & -.134 & .002 & -.040 & .238 & .051 & -.116 \\
\hline .575 & -.078 & .184 & .090 & .009 & -.044 & .317 \\
\hline .571 & -.328 & .354 & .149 & -.133 & -.058 & .035 \\
\hline .554 & .274 & -.119 & .099 & .191 & .192 & .038 \\
\hline .490 & -.488 & .213 & -.031 & -.072 & -.151 & .024 \\
\hline .490 & .014 & -.255 & -.169 & -.205 & .214 & .093 \\
\hline .469 & -.064 & .193 & .284 & -.193 & .022 & .010 \\
\hline .457 & -.141 & .256 & .075 & .102 & .072 & .164 \\
\hline .433 & .218 & .053 & -.137 & -.345 & .219 & -.110 \\
\hline .432 & .142 & -.303 & .342 & -.035 & .033 & -.048 \\
\hline .431 & .123 & .030 & .322 & -.122 & .155 & .017 \\
\hline .419 & .278 & .225 & -.081 & .129 & .035 & .109 \\
\hline
\end{tabular}

$\begin{array}{lllllll}.403 & .230 & .060 & -.091 & -.148 & .127 & -.024\end{array}$ $\begin{array}{llllllll}.392 & .201 & -.228 & .348 & .184 & -.203 & -.004\end{array}$ 
It is unacceptable to bend the truth on the Internet to get the information you want out of a woman. (RC)

Even if I can, I am not free to bypass online. (HC)

It is not appropriate to forge identifying information of a woman on the Internet to learn more about her. (IM)

Just because someone is telling a woman what they think, it does not mean it is threatening or intimidating. (HS)

It is unacceptable to use the Internet to trace where a woman has been. (ST) security to get information about a woman

\begin{tabular}{lllllll}
.369 & -.001 & -.108 & .260 & -.029 & -.118 & .024 \\
.362 & .093 & .154 & .129 & .067 & .201 & .095 \\
& & & & & & \\
.325 & .266 & -.181 & .303 & .189 & .049 & .011 \\
& & & & & & \\
.345 & -.373 & .063 & .040 & .348 & .072 & -.161 \\
& & & & & & \\
.422 & -.030 & .149 & .452 & -.249 & .022 & -.130 \\
& & & & & & \\
\hline
\end{tabular}

Note . $(\mathrm{HC})=$ Hacking; $(\mathrm{IM})=$ Impersonation; $(\mathrm{ST})=$ Surveillance \& Tracking; $(\mathrm{HS})=$ Harassment \& Spamming; (RC) = Recruitment; (MD) = Malicious Distribution. 


\section{Appendix L - Factor Loadings Table for the first PAF conducted in Study 3}

\begin{tabular}{|c|c|c|c|c|c|c|c|}
\hline \multirow[b]{2}{*}{ Item } & \multicolumn{7}{|c|}{ Factor } \\
\hline & 1 & 2 & 3 & 4 & 5 & 6 & 7 \\
\hline $\begin{array}{l}\text { There is nothing wrong with using fake } \\
\text { advertisements or profiles to trick a woman } \\
\text { online. (RC) }\end{array}$ & .675 & -.106 & -.179 & -.005 & -.275 & .108 & -.051 \\
\hline $\begin{array}{l}t \text { is okay to trick a woman into a } \\
\text { RC) }\end{array}$ & .671 & -.082 & -.151 & -.053 & -.224 & .040 & -.076 \\
\hline $\begin{array}{l}\text { 's okay to create new, anonymous online } \\
\text { rofiles or accounts for the sole purpose of } \\
\text { sing them to spam a woman with } \\
\text { essages. (HS) }\end{array}$ & .649 & -.027 & -.172 & -.008 & -.019 & -.061 & -.101 \\
\hline lead a woman online. (RC) & .627 & .104 & -.124 & .042 & -.291 & .198 & .118 \\
\hline $\begin{array}{l}\text { o ge } \\
\text { kay }\end{array}$ & .618 & -.474 & .013 & -.258 & .177 & .053 & .064 \\
\hline $\begin{array}{l}\text { There's nothing wrong with making } \\
\text { unprovoked sexual remarks towards or } \\
\text { sexual requests of a woman on the Internet. } \\
\text { (HS) }\end{array}$ & .617 & .210 & -.244 & .006 & -.059 & -.039 & -.131 \\
\hline $\begin{array}{l}\text { is okay to impersonate someone online to } \\
\text { arn more about a woman. (IM) }\end{array}$ & .613 & -.292 & .025 & -.146 & .084 & .164 & .063 \\
\hline $\begin{array}{l}\text { f I have figured out the password to a } \\
\text { voman's account or profile, I can do what I } \\
\text { vant with it. (IM) }\end{array}$ & .591 & -.323 & .012 & -.215 & .109 & -.012 & .053 \\
\hline $\begin{array}{l}\text { t is okay to share intimate photos or videos } \\
\text { f a woman online without her consent. } \\
\text { MD) }\end{array}$ & .572 & -.165 & -.075 & -.068 & -.005 & -.079 & -.047 \\
\hline $\begin{array}{l}\text { Continually monitoring the activities of a } \\
\text { voman online is totally fine. (ST) }\end{array}$ & .527 & .137 & .181 & -.113 & -.058 & -.036 & -.025 \\
\hline $\begin{array}{l}\text { It is fine to use the Internet to contact a } \\
\text { woman even though she does not want to } \\
\text { be contacted. (HS) }\end{array}$ & .526 & .176 & -.076 & -.012 & .094 & -.021 & -.028 \\
\hline $\begin{array}{l}\text { Spamming a woman's e-mail, social media, } \\
\text { phone or voicemail with messages is } \\
\text { wrong. (HS) }\end{array}$ & .503 & -.024 & -.083 & .176 & .059 & -.168 & -.124 \\
\hline $\begin{array}{l}\text { ceptable to use the Internet to trace } \\
\text { voman has been. (ST) }\end{array}$ & .498 & .210 & . 476 & .029 & -.070 & -.128 & -.108 \\
\hline $\begin{array}{l}\text { On the Internet, to convince a woman of } \\
\text { something, sometimes you have to say } \\
\text { things that are not entirely true. (RC) }\end{array}$ & .484 & .064 & -.061 & -.020 & -.191 & .126 & .109 \\
\hline $\begin{array}{l}\text { Twisting the truth about a woman online for } \\
\text { my personal purposes is not right. (MD) }\end{array}$ & .479 & -.160 & -.019 & .302 & -.062 & -.222 & .304 \\
\hline
\end{tabular}


There's nothing wrong with threatening real life violence to a woman on the Internet. (HS)

I'm at liberty to send intimate photos of myself to any woman I choose. (MD) There is no problem with telling a woman to kill herself on the Internet. (HS)

There is nothing wrong with using the Internet to trace a woman's history. (ST) It is not okay to use the Internet to track down a woman. (ST)

Obtaining a woman's personal information online without her permission is never okay. (HC)

I do not have the right to spread fake stories or rumors about a woman online. (MD)

If I want to access information about a woman online, I can do what I need to do to acquire it. (HC)

Pretending to be someone else when contacting a woman on the Internet is not okay. (IM)

It is acceptable to gain unauthorized access to a woman's information on the Internet.

(HC)

It is okay to gain access to a live stream of a woman's webcam without her knowledge.

(HC)

It is not okay to use GPS or location

tracking to find out where a woman is right now. (ST)

There is potential harm in repeatedly messaging, calling or texting a woman. (HS)

Even if I can, I am not free to bypass security to get information about a woman online. (HC)

Threatening to release private information about a woman onto the Internet in order to get my way is acceptable thing to do. (MD) It is unacceptable to bend the truth on the Internet to get the information you want out of a woman. (RC)

It is okay to use the Internet to tell a woman what I think about her, regardless of if she wants to know or not. (HS) $\begin{array}{lllllll}.476 & -.069 & -.243 & -.018 & -.025 & -.093 & -.148\end{array}$

\begin{tabular}{|c|c|c|c|c|c|}
\hline $\begin{array}{ll}465 & .189\end{array}$ & -.121 & .014 & .072 & -.055 & -.055 \\
\hline .167 & -.253 & .030 & .019 & -.070 & -.060 \\
\hline .348 & .380 & -.190 & -.104 & -.001 & .017 \\
\hline .082 & .398 & .097 & -.036 & -.058 & -.094 \\
\hline .000 & .218 & -.053 & .043 & -.004 & .055 \\
\hline .039 & -.060 & .260 & .004 & -.184 & .330 \\
\hline .012 & .170 & -.112 & .053 & .005 & .063 \\
\hline $00 \quad-.106$ & .104 & .387 & .095 & .351 & -.074 \\
\hline $99-.171$ & .066 & -.166 & .093 & -.025 & .052 \\
\hline
\end{tabular}

$\begin{array}{lllllll}.399 & -.183 & .035 & -.170 & .105 & -.036 & .031\end{array}$

$\begin{array}{llllllll}.393 & -.062 & .302 & .185 & .032 & -.087 & -.108 \\ & & & & & & \\ .388 & .140 & -.123 & .099 & .111 & -.149 & -.107\end{array}$

$\begin{array}{lllllll}.384 & -.211 & .083 & .033 & .075 & -.024 & .023\end{array}$

$\begin{array}{llllllll}.322 & -.151 & -.067 & -.063 & .010 & -.035 & -.075\end{array}$

$\begin{array}{lllllll}.315 & .033 & .071 & .183 & -.031 & .055 & .141\end{array}$

$\begin{array}{lllllll}.422 & .441 & -.177 & -.018 & .250 & .080 & .042\end{array}$ 
I have the right to express my anger towards a woman online as frequently as I wish. (HS)

If a woman has an online presence, it is okay for me to use information she puts out there to learn more about her. (ST)

Just because someone is telling a woman what they think, it does not mean it is threatening or intimidating. (HS)

It is not appropriate to forge identifying information of a woman on the Internet to learn more about her. (IM)

Note . $(\mathrm{HC})=$ Hacking; $(\mathrm{IM})=$ Impersonation; $(\mathrm{ST})=$ Surveillance \& Tracking; $(\mathrm{HS})=$ Harassment \& Spamming; (RC) = Recruitment; (MD) = Malicious Distribution.

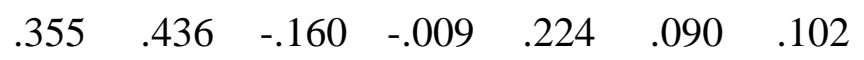

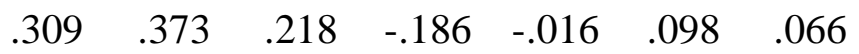

$\begin{array}{lllllll}.108 & .336 & -.093 & -.047 & .140 & .084 & .058\end{array}$

$\begin{array}{lllllll}.342 & -.204 & .135 & .409 & .156 & .183 & -.117\end{array}$ 


\section{References}

Abrams, D., Viki, G. T., Masser, B., \& Bohner, G. (2003). Perceptions of stranger and acquaintance rape: Role of benevolent and hostile sexism in victim blame and rape proclivity. Journal of Personality and Social Psychology, 84(1), 111-125. http://doi.org/10.1037/0022-3514.84.1.111

Adorno, T. W., Frenkel-Brunswik, E., Levinson, D. J., \& Sanford, R. N. . (1950). The authoritarian personality. New York: Harpers.

Altemeyer, B. (1981). Right-wing authoritarianism. Winnipeg, Manitoba, Canada: University of Manitoba Press.

Altemeyer, B. (1988). Enemies of freedom: Understanding right-wing authoritarianism. San Francisco, CA: Jossey-Bass. Retrieved from http://search.ebscohost.com/login.aspx?direct=true\&db=psyh\&AN=1988-98419000\&site $=$ ehost-live

Altemeyer, B. (1996). The authoritarian specter. Cambridge, MA: Harvard University Press. Altemeyer, B. (2006). The Authoritarians, 1-261.

Baker, A. L., Campbell, M., \& Baretto, E. (2013). Understanding Technology-Related Violence Against Women: Types of Violence and Women's Experiences. London, Ontario. Retrieved from http://www.vawlearningnetwork.ca/understanding-technology-related-violenceagainst-women-types-violence-and-womens-experiences

Barak, A. (2005). Sexual Harassment on the Internet. Social Science Computer Review, 23(1), 77-92. http://doi.org/10.1177/0894439304271540

Barlett, C., \& Coyne, S. M. (2014). A Meta-Analysis of Sex Differences in Cyber - Bullying Behavior: The Moderating Role of Age, 40(April), 474-488. 
http://doi.org/10.1002/ab.21555

Bates, C., \& Heaven, P. C. L. (2001). Attitudes to women in society: The role of social dominance orientation and social values. Journal of Community \& Applied Social Psychology, 11(November 1999), 43-49. http://doi.org/10.1002/casp.589.abs

Beere, C., King, D., Beere, D., \& King, L. (1984). The Sex-Role Egalitarianism Scale: A Measure of Attitudes Toward Equality Between the Sexes. Sex Roles 1, 10(7). http://doi.org/10.1017/CBO9781107415324.004

Behm-Morawitz, E., \& Schipper, S. (2015). Sexing the Avatar. Journal of Media Psychology, 114. http://doi.org/10.1027/1864-1105/a000152

Berne, S., Frisén, a., Schultze-Krumbholz, A., Scheithauer, H., Naruskov, K., Luik, P., ... Zukauskiene, R. (2013). Cyberbullying assessment instruments: A systematic review. Aggression and Violent Behavior, 18(2), 320-334. http://doi.org/10.1016/j.avb.2012.11.022

Branscombe, N. R., \& Ellemers, N. (1998). Coping with group-based discrimination:

Individualistic versus group-level strategies. In The target perspective (pp. 243-266). Retrieved from http://search.ebscohost.com/login.aspx?direct=true\&db=psyh\&AN=199807621-011\&site=ehost-live \&scope=site

Branscombe, N. R., Ellemers, N., Spears, R., \& Doosje, B. (1999). The context and content of social identity threat. In Social identity: Context, commitment, content (pp. 35-58). http://doi.org/MAI HM131 S58433 1999

Branscombe, N. R., \& Wann, D. (1994). Collective self-esteem consequences of outgroup derogation when a valued social identity is on trial. European Journal of Social Psychology, 24(6), 641-657.

Bustillos, A., \& Silván-ferrero, M. P. (2013). Attitudes Toward Peers With Physical Disabilities 
at High School: Applying the Integrated Threat Theory. http://doi.org/10.1177/0034355212451145

Calvete, E., Orue, I., Estévez, A., Villardón, L., Padilla, P., Estévez, A., ... Padilla, P. (2010). Cyberbullying in adolescents: Modalities and aggressors' profile. Computers in Human Behavior, 26(5), 1128-1135. http://doi.org/10.1016/j.chb.2010.03.017

Cappadocia, M. C., Craig, W. M., \& Pepler, D. (2013). Cyberbullying Prevalence, Stability, and Risk Factors During Adolescence. Canadian Journal of School Psychology, 28(2), 171192. http://doi.org/10.1177/0829573513491212

Carbone-Lopez, K., Esbensen, F. -a., \& Brick, B. T. (2010). Correlates and Consequences of Peer Victimization: Gender Differences in Direct and Indirect Forms of Bullying. Youth Violence and Juvenile Justice, 8(4), 332-350. http://doi.org/10.1177/1541204010362954

Charles-Toussaint, G. C., \& Crowson, H. M. (2010). Prejudice against international students: the role of threat perceptions and authoritarian dispositions in U.S. students. The Journal of Psychology, 144(5), 413-428. http://doi.org/10.1080/00223980.2010.496643

Charlesford, J., \& Choma, B. L. (2013). Intergroup threat scale.

Chisholm, J. F. (2006a). Cyberspace Violence against Girls and Adolescent Females, 89, 74-89. http://doi.org/10.1196/annals.1385.022

Chisholm, J. F. (2006b). Cyberspace Violence Against Girls and Adolescent Females. Annals of the New York Academy of Sciences, 1087, 74-89. http://doi.org/10.1196/annals.1385.022

Choma, B. L., Haji, R., Hodson, G., \& Hoffarth, M. (2016). Avoiding cultural contamination: Intergroup disgust sensitivity and religious identification as predictors of interfaith threat, faith-based policies, and islamophobia. Personality and Individual Differences, 95, 50-55. http://doi.org/10.1016/j.paid.2016.02.013 
Cleemput, V., \& Grigg, D. W. (2010). Cyber-Aggression: Definition and Concept of Cyberbullying. Australian Journal of Guidance and Counselling, 20(2), 143-156. http://doi.org/10.1375/ajgc.20.2.143

Cober, A., Ritter, B. A., Biber, J. K., Doverspike, D., Baznik, D., Cober, A., \& Ritter, B. A. (2002). Sexual harassment in online communications: Effects of gender and discourse medium. Cyberpsychology \& Behavior: The Impact of the Internet, Multimedia and Virtual Reality on Behavior and Society, 5(1), 33-42. http://doi.org/10.1089/109493102753685863

Cohrs, C. (2013). Threat and authoritarianism: some theoretical and methodological comments. International Journal of Psychology: Journal International de Psychologie, 48(1), 50-4. http://doi.org/10.1080/00207594.2012.732699

Cohrs, C., \& Asbrock, F. (2009). Right-wing authoritarianism, social dominance orientation and prejudice against threatening and competitive ethnic groups. European Journal of Social Psychology, 39(2), 270-289. http://doi.org/10.1002/ejsp.545

Connor, R. A., Glick, P., \& Fiske, S. T. (2017). Ambivalent Sexism in the Twenty-First Century. In C. G. Sibley \& F. K. Barlow (Eds.), The Cambridge Handbook of The Psychology of Prejudice (pp. 295-320). Cambridge, UK: Cambridge University Press.

Costello, A. B., \& Osborne, J. W. (2005). Best Practices in Exploratory Factor Analysis : Four Recommendations for Getting the Most From Your Analysis.

Craig, M. A., \& Richeson, J. A. (2014). Not in my backyard! authoritarianism, social dominance orientation, and support for strict immigration policies at home and abroad. Political Psychology, 35(3), 417-429. http://doi.org/10.1111/pops.12078

Crowson, H. M. (2009). Predicting perceptions of symbolic and realistic threat from terrorists: The role of right-wing authoritarianism and social dominance orientation. Individual 
Differences Research, 7(2), 113-118.

Davies, M. (2004). Correlates of negative attitudes toward gay men: sexism, male role norms, and male sexuality. Journal of Sex Research, 41(3), 259-266.

http://doi.org/10.1080/00224490409552233

Dehue, F., Bolman, C., \& Völlink, T. (2008). Cyberbullying: youngsters' experiences and parental perception. Cyberpsychology \& Behavior : The Impact of the Internet, Multimedia and Virtual Reality on Behavior and Society, 11(2), 217-223.

http://doi.org/10.1089/cpb.2007.0008

Dhont, K., Roets, A., \& Van Hiel, A. (2013). The intergenerational transmission of need for closure underlies the transmission of authoritarianism and anti-immigrant prejudice. Personality and Individual Differences, 54(6), 779-784. http://doi.org/10.1016/j.paid.2012.12.016

Dhont, K., \& Van Hiel, A. (2012). Intergroup contact buffers against the intergenerational transmission of authoritarianism and racial prejudice. Journal of Research in Personality, 46(2), 231-234. http://doi.org/10.1016/j.jrp.2011.12.008

Dooley, J. J., Pyżalski, J., \& Cross, D. (2009). Cyberbullying Versus Face-to-Face Bullying. Zeitschrift Für Psychologie / Journal of Psychology, 217(4), 182-188.

http://doi.org/10.1027/0044-3409.217.4.182

Dru, V. (2007). Authoritarianism, social dominance orientation and prejudice: Effects of various self-categorization conditions. Journal of Experimental Social Psychology, 43(6), 877-883. http://doi.org/10.1016/j.jesp.2006.10.008

Duckitt, J. (2001). A dual-process cognitive-motivational theory of ideology and prejudice. In Advances in Experimental Social Psychology (33rd ed., pp. 41-113). San Diego, CA: 
Academic Press.

Duckitt, J. (2004). The cultural bases of ethnocentrism: Comparing White Afrikaners and European New Zealanders. In Y.-T. Lee, C. McCauley, F. Moghaddam, \& S. Worchel (Eds.), The psychology of ethnic and cultural conflict (1st ed., pp. 155-173). Westport, CT: Praeger Publishers.

Duckitt, J. (2006). Differential Effects of Right Wing Authoritarianism and Social Dominance Orientation on Outgroup Attitudes and Their Mediation by Threat From and Competitiveness to Outgroups. Personality and Social Psychology Bulletin, 32(5), 684-696. http://doi.org/10.1177/0146167205284282

Duckitt, J., \& Sibley, C. G. (2007). Right Wing Authoritarianism, Social Dominance Orientation and The Dimensions of Generalized Prejudice. European Journal of Personality, 21(2), 113-130. http://doi.org/10.1002/per.614

Duckitt, J., \& Sibley, C. G. (2009). A Dual-Process Motivational Model of Ideology, Politics, and Prejudice. Psychological Inquiry, 20(2-3), 98-109. http://doi.org/10.1080/10478400903028540

Duriez, B., \& Soenens, B. (2009). The intergenerational transmission of racism: The role of Right-Wing Authoritarianism and Social Dominance Orientation. Journal of Research in Personality, 43(5), 906-909. http://doi.org/10.1016/j.jrp.2009.05.014

Duriez, B., Soenens, B., \& Vansteenkiste, M. (2008). The intergenerational transmission of authoritarianism: The mediating role of parental goal promotion. Journal of Research in Personality, 42(3), 622-642. http://doi.org/10.1016/j.jrp.2007.08.007

Eagly, A. H., \& Mladinic, A. (1989). Gender stereotypes and attitudes toward women and men. Personality and Social Psychology Bulletin. http://doi.org/10.1177/0146167289154008 
Ekehammar Akrami, N., Gylje,M., Zakrisson, I., B. (2004). What matters most to perjudice: big five personality, social dominance orientation, or right-wing authoritarianism ? European Journal of Personality, 18(October 2003), 463-482. http://doi.org/10.1002/per.526

Entertainment Software Association. (2007). Essential facts about the computer and video game industry. Washington, DC. Retrieved from http://essentialfacts.theesa.com/Essential-Facts2016.pdf

Entertainment Software Association. (2014). Essential Facts About the Computer and Video Game Industry. Washington, DC. Retrieved from http://www.theesa.com/wpcontent/uploads/2014/10/ESA_EF_2014.pdf

Esses, V. M., Dovidio, J. F., Jackson, L. M., \& Armstrong, T. L. (2001). The Immigration Dilemma: The Role of Perceived Group Competition, Ethnic Prejudice, and National Identity. Journal of Social Issues, 57(3), 389-412. http://doi.org/10.1111/0022-4537.00220

European Union Agency for Fundamental Rights. (2014). Violence against women : An EU-wide survey. http://doi.org/10.2811/62230

Fabrigar, L. R., Wegener, D. T., Maccallum, R. C., \& Strahan, E. J. (1999). Evaluating the Use of Exploratory Factor Analysis in Psychological Research. Psychological Methods, 4(3), 272-299. http://doi.org/10.1037/1082-989X.4.3.272

Feldman, S., \& Stenner, K. (1997). Perceived Threat and Authoritarianism. Political Psychology, 18(4), 741-770. http://doi.org/10.1111/0162-895X.00077

Fichman, P., \& Sanfilippo, M. R. (2015). The Bad Boys and Girls of Cyberspace: How Gender and Context Impact Perception of and Reaction to Trolling. Social Science Computer Review, 33(2), 163-180. http://doi.org/10.1177/0894439314533169

Forero, R., Mclellan, L., Rissel, C., \& Bauman, A. (n.d.). Bullying behaviour and psychosocial 
health among school students in New South Wales, Australia : cross sectional survey.

Fox, J., \& Tang, W. (2014). Computers in Human Behavior Sexism in online video games: The role of conformity to masculine norms and social dominance orientation. Computers in Human Behavior, 33, 314-320. http://doi.org/10.1016/j.chb.2013.07.014

Glick, P., \& Fiske, S. T. (1996). The Ambivalent Sexism Inventory: Differentiating Hostile and Benevolent Sexism, 70(3), 491-512. http://doi.org/10.1037/0022-3514.70.3.491

Glick, P., \& Fiske, S. T. (1997). Hostile and Benevolent Sexism. Psychology of Women Quarterly, 21(1), 119-135. http://doi.org/10.1111/j.1471-6402.1997.tb00104.x

Glick, P., \& Fiske, S. T. (2001). An Ambivalent Alliance: Hostile and Benevolent Sexism as Complementary Justifications for Gender Inequality. American Psychologist, 56(2), 109118. http://doi.org/10.1037//O003-066X.56.2.1O9

Glick, P., Fiske, S. T., Mladinic, A., Saiz, J. L., Abrams, D., Masser, B., ... López López, W. (2000). Beyond Prejudice as Simple Antipathy : Hostile and Benevolent Sexism Across Cultures. Journal of Personality and Social Psychology, 79(5), 763-775. http://doi.org/10.1037/0022-3514.79.5.763

Goertzel, T. G. (1987). Authoritarianism of personality and political attitudes. Journal of Social Psychology, 127(1), 7-18.

Grundberg, S., \& Hansegard, J. (2014). Women Now Make Up Almost Half of Gamers. Retrieved May 2, 2017, from https://www.wsj.com/articles/gaming-no-longer-a-mansworld-1408464249

Guo, S. (2016). A Meta-Analysis of the Predictors of Cyberbullying Perpetration and Victimization. Psychology in the Schools, 53(4), 432-453. http://doi.org/10.1002/pits.21914 Henry, N., \& Powell, A. (2015). Embodied Harms: Gender, Shame, and Technology-Facilitated 
Sexual Violence. Violence Against Women, 21(6), 758-779.

http://doi.org/10.1177/1077801215576581

Ho, A. K., Sidanius, J., Kteily, N., Sheehy-Skeffington, J., Pratto, F., Henkel, K. E., ... Stewart, A. L. (2015). The Nature of Social Dominance Orientation: Theorizing and Measuring Preferences for Intergroup Inequality Using the New SDO 7 Scale. Journal of Personality and Social Psychology, 109(6), 1003-1028. http://doi.org/10.1037/pspi0000033

Internet Advertising Bureau UK. (2014). More women now play video games than men. London, Ontario. Retrieved from http://www.iabuk.net/about/press/archive/more-women-now-playvideo-games-than-men

Jackson, D. L. (2003). Revisiting Sample Size and Number of Parameter Estimates: Some Support for the N:q Hypothesis. Structural Equation Modeling, 10(1), 1-34. http://doi.org/10.1207/S15328007SEM1001

Jost, J. T., Glaser, J., Kruglanski, A. W., \& Sulloway, F. J. (2003). Political conservatism as motivated social cognition. Psychological Bulletin, 129(3), 339-375. http://doi.org/10.1037/0033-2909.129.3.339

Jost, J. T., Noorbaloochi, S., \& Van Bavel, J. J. (2014). The "chicken-and-egg" problem in political neuroscience. Behavioral and Brain Sciences, 37(3), 317-318. http://doi.org/10.1017/S0140525X13001192

Kilpatrick, D. G. (2004). What is violence against women: defining and measuring the problem. Journal of Interpersonal Violence, 19(11), 1209-34. http://doi.org/10.1177/0886260504269679

Kowalski, R. M., Giumetti, G. W., Schroeder, A. N., \& Lattanner, M. R. (2014). Bullying in the digital age: A critical review and meta-analysis of cyberbullying research among youth. 
Psychological Bulletin, 140(4), 1073-1137. http://doi.org/10.1037/a0035618

Levin, S. (2004). Perceived Group Status Differences and the Effects of Gender , Ethnicity, and Religion on Social. Political Psychology, 25(1), 31-48.

Li, Q. (2007). New bottle but old wine: A research of cyberbullying in schools. Computers in Human Behavior, 23(4), 1777-1791. http://doi.org/10.1016/j.chb.2005.10.005

Lippa, R., \& Arad, S. (1999). Gender, Personality, and Prejudice: The Display of Authoritarianism and Social Dominance in Interviews with College Men and Women. Journal of Research in Personality, 33(4), 463-493. http://doi.org/10.1006/jrpe.1999.2266

Luhtanen, R., \& Crocker, J. (1992). A collective self-esteem scale: Self-evaluation of one's social identity. Personality and Social Psychology Bulletin, 18(3), 302-318.

Matthews, M., Levin, S., \& Sidanius, J. (2009). A longitudinal test of the model of political conservatism as motivated social cognition. Political Psychology, 30(6), 921-936. http://doi.org/10.1111/j.1467-9221.2009.00733.x

McConahay, J. B. (1986). Modern racism, ambivalence, and the modern racism scale. In Prejudice, Discrimination, and Racism (pp. 91-125). San Diego, CA: Academic Press.

McFarland, S. G. (2005). On the eve of war: authoritarianism, social dominance, and American students' attitudes toward attacking Iraq. Personality and Social Psychology Bulletin, 31(3), 360-367. http://doi.org/10.1177/0146167204271596

McHugh, M. C., \& Frieze, I. H. (1997). THE MEASUREMENT OF GENDER-ROLE ATTITUDES A Review and Commentary. Psychology of Women Quarterly, 21(1), 1-16. http://doi.org/10.1111/j.1471-6402.1997.tb00097.x

Meeusen, C., \& Dhont, K. (2015). Parent-Child Similarity in Common and Specific Components of Prejudice: The Role of Ideological Attitudes and Political Discussion. European Journal 
of Personality, 598(July), 585-598. http://doi.org/10.1002/per.2011

Mirisola, A., \& Russo, S. (2014). Societal Threat to Safety , Compensatory Control, and RightWing Authoritarianism, 35(6). http://doi.org/10.1111/pops.12048

Morrison, K. R., \& Ybarra, O. (2008). The effects of realistic threat and group identification on social dominance orientation. Journal of Experimental Social Psychology, 44(1), 156-163. http://doi.org/10.1016/j.jesp.2006.12.006

Morrison, K. R., \& Ybarra, O. (2009). Symbolic threat and social dominance among liberals and conservatives: SDO reflects conformity to political values. European Journal of Social Psychology, 39(6), 1039-1052. http://doi.org/10.1002/ejsp

Nansel, T. R., Overpeck, M., Pilla, R. S., Ruan, W. J., Simons-Morton, B., \& Scheidt, P. (2001). Bullying behaviors among US youth: prevalence and association with psychosocial adjustment. Jama, 285(16), 2094-100. http://doi.org/10.1001/jama.285.16.2094

Olweus, D. (1993). Bullying at School: What We Know and What We Can Do. Cambridge, MA: Wiley-Blackwell.

Ouwerkerk, J. W., de Gilder, D., de Vries, N. K., Gilder, D. De, \& Vries, N. K. De. (2000). When the going gets tough, the tough get going: Social identification and individual effort in intergroup competition. Personality and Social Psychology Bulletin, 26(12), 1550-1559. http://doi.org/10.1177/01461672002612009

Parkins, I. S., Fishbein, H. D., \& Ritchey, P. N. (2006). The Influence of Personality and Social motivation on Workplace Bullying and Discrimination. Journal of Applied Social Psychology, 36(10), 2554-2577.

Pratto, F. (1999). The Puzzle of Continuing Group Inequality: Piecing Together Psychological, Social, and Cultural Forces in Social Dominance Theory. Advances in Experimental Social 
Psychology, 31(C), 191-263. http://doi.org/10.1016/S0065-2601(08)60274-9

Pratto, F., Liu, J. H., Levin, S., Sidanius, J., Shih, M., Bachrach, H., \& Hegarty, P. (2000). Social dominance orientation and the legitimization of inequality across cultures. Journal of CrossCultural Psychology, 31(3), 369-409. http://doi.org/10.1177/0022022100031003005

Pratto, F., Sidanius, J., \& Levin, S. (2006). Social dominance theory and the dynamics of intergroup relations: Taking stock and looking forward. European Review of Social Psychology, 17(1), 271-320. http://doi.org/10.1080/10463280601055772

Pratto, F., Sidanius, J., Stallworth, L. M., \& Malle, B. F. (1994). Social dominance orientation: A personality variable predicting social and political attitudes. Journal of Personality and Social Psychology, 67(4), 741-763. http://doi.org/10.1037/0022-3514.67.4.741

Pratto, F., \& Walker, A. (2004). The Bases of Gendered Power. In The psychology of gender (pp. 242-268).

Riek, B. M., Mania, E. W., \& Gaertner, S. L. (2006). Intergroup Threat and Outgroup Attitudes : A Meta-Analytic Review, 10(4), 336-353. http://doi.org/10.1016/j.ijintrel.2005.08.003

Rios, K. (2013). Journal of Experimental Social Psychology Right-wing authoritarianism predicts prejudice against " homosexuals" but not " gay men and lesbians ." Journal of Experimental Social Psychology, 49(6), 1177-1183. http://doi.org/10.1016/j.jesp.2013.05.013

Roccato, M., \& Ricolfi, L. (2005). On the Correlation Between Right-Wing Authoritarianism and Social Dominance Orientation. Basic and Applied Social Psychology, 27(3), 187-200. http://doi.org/10.1207/s15324834basp2703_1

Rollero, C., Glick, P., \& Tartaglia, S. (2014). Psychometric properties of short versions of the Ambivalent Sexism Inventory and Ambivalence Toward Men Inventory. TPM - Testing, 
Psychometrics, Methodology in Applied Psychology, 21(2), 149-159.

http://doi.org/10.4473/TPM21.2.3

Ruffman, T., Wilson, M., Henry, J. D., Dawson, A., Chen, Y., Kladnitski, N., ... Hunter, J. A. (2016). Age Differences in Right-Wing Authoritarianism and Their Relation to Emotion Recognition, 16(2), 226-236.

Russell, B. L., \& Trigg, K. Y. (2004). Tolerance of Sexual Harassment : An Examination of Gender Differences , ..., 50(April).

Sears, D. O. (1988). Symbolic racism. In P. A. Katz \& D. A. Taylor (Eds.), Eliminating racism: Profiles in controversy (pp. 53-84). New York, NY: Plenum Press.

Sherif, M., \& Sherif, C. W. (1969). Ingroup and intergroup relations: Experimental analysis. In M. Sherif \& C. W. Sherif (Eds.), Social Psychology (pp. 221-266). New York, NY: Harper \& Row.

Sibley, C. G., Robertson, A., \& Wilson, M. S. (2006). Social Dominance Orientation and RightWing Authoritarianism: Additive and Interactive Effects. Political Psychology, 27(5), 755768. http://doi.org/10.1111/j.1467-9221.2006.00531.x

Sibley, C. G., Wilson, M. S., \& Duckitt, J. (2007). Antecedents of Men's Hostile and Benevolent Sexism: The Dual Roles of Social Dominance Orientation and Right-Wing Authoritarianism. Personality and Social Psychology Bulletin, 33(2), 160-172. http://doi.org/10.1177/0146167206294745

Sidanius, J., Bobo, L., Pratto, F., \& Bobo, L. (1996). Racism, conservatism, Affirmative Action, and intellectual sophistication: A matter of principled conservatism or group dominance? Journal of Personality and Social Psychology, 70(3), 476-490. http://doi.org/10.1037/00223514.70.3.476 
Sidanius, J., Cotterill, S., Sheehy-Skeffington, J., Kteily, N., \& Carvacho, H. (2017). Social Dominance Theory: Explorations in the Psychology of Oppression. In C. G. Sibley \& F. K. Barlow (Eds.), The Cambridge Handbook of The Psychology of Prejudice (pp. 149-187). Cambridge, UK: Cambridge University Press.

Sidanius, J., Levin, S., Liu, J., \& Pratto, F. (2000). Social dominance orientation, antiegalitarianism and the political psychology of gender: an extension and cross-cultural replication. European Journal of Social Psychology, 30(1), 41-67. http://doi.org/10.1002/(SICI)1099-0992(200001/02)30:1<41::AID-EJSP976>3.0.CO;2-O

Sidanius, J., \& Pratto, F. (1999). Social dominance: An intergroup theory of social hierarchy and oppression. Cambridge, UK: Cambridge University Press. http://doi.org/10.2307/2655372

Smith, P. K., Mahdavi, J., Carvalho, M., Fisher, S., Russell, S., \& Tippett, N. (2008). Cyberbullying: Its nature and impact in secondary school pupils. Journal of Child Psychology and Psychiatry, 49(4), 376-385. http://doi.org/10.1111/j.14697610.2007.01846.x

Spence, J. T., \& Helmreich, R. (1972). Who Likes Competent Women? Competence, Sex-Role Congruence of Interests, and Subjects' Attitudes Toward Women as Determinants of Interpersonal Attraction. Journal of Applied Social Psychology, 2(3), 197-213. http://doi.org/10.1111/j.1559-1816.1972.tb01272.x

Stephan, W. G. (2014). Intergroup Anxiety: Theory, Research, and Practice. Personality and Social Psychology Review : An Official Journal of the Society for Personality and Social Psychology, Inc, 18(3), 239-255. http://doi.org/10.1177/1088868314530518

Stephan, W. G., \& Stephan, C. W. (1985). Intergroup anxiety. Journal of Social Issues, 41(3), 157-175. http://doi.org/10.1111/j.1540-4560.1985.tb01134.x 
Stephan, W. G., \& Stephan, C. W. (2000). An integrated threat theory of prejudice. In Reducing prejudice and discrimination (pp. 23-45).

Stephan, W. G., Ybarra, O., \& Bachman, G. (1999). Prejudice toward immigrants. Journal of Applied Social Psychology, 29(11), 2221-2237. http://doi.org/10.1111/j.1559-

1816.1999.tb00107.x

Stephan, W. G., Ybarra, O., Martnez, C. M., Schwarzwald, J., \& Tur-Kaspa, M. (1998).

Prejudice toward Immigrants to Spain and Israel: An Integrated Threat Theory Analysis. Journal of Cross-Cultural Psychology. http://doi.org/10.1177/0022022198294004

Stone, W. F., Lederer, G., \& Christie, R. (1993). Strength and weakness: The authoritarian personality today. New York, NY: Springer-Verlag Publishing.

Swim, J. K., Aikin, K. J., Hall, W. S., \& Hunter, B. A. (1995). Sexism and Racism : OldFashioned and Modern Prejudices, 68(2), 199-214.

Tajfel, H., \& Turner, J. C. (1986). The social identity theory of intergroup behavior. In Psychology of Intergroup Relations (Vol. 2nd ed., pp. 7-24). http://doi.org/10.1111/j.17519004.2007.00066.x

Tang, W. Y., \& Fox, J. (2016). Men's harassment behavior in online video games: Personality traits and game factors. Aggressive Behavior, 9999, 1-9. http://doi.org/10.1002/ab.21646

Thomas, H. J., Connor, J. P., \& Scott, J. G. (2015). Integrating Traditional Bullying and Cyberbullying : Challenges of Definition and Measurement in Adolescents - a Review. Educational Psychology Review, 27(1), 135-152. http://doi.org/10.1007/s10648-014-9261-7

Tokunaga, R. S. (2010). Following you home from school: A critical review and synthesis of research on cyberbullying victimization. Computers in Human Behavior, 26(3), 277-287. http://doi.org/10.1016/j.chb.2009.11.014 
Turner, J. C., Brown, R. J., \& Tajfel, H. (1979). Social comparison and group interest in ingroup favouritism. European Journal of Social Psychology, 9(February 1978), 187-204. http://doi.org/10.1002/ejsp.2420090207

Uenal, F. (2016). Disentangling Islamophobia: The Differential Effects of Symbolic, Realistic, and Terroristic Threat Perceptions as Mediators Between Social Dominance Orientation and Islamophobia. Journal of Social and Political Psychology, 4(1), 66-90. http://doi.org/10.5964/jspp.v4i1.463

United Nations Entity for Gender Equality and the Empowerment of Women and United Nations Development Programme. (2015). Cyber Violence Against Women and Girls. Retrieved from http://www.unwomen.org/en/digital-library/publications/2015/9/cyber-violenceagainst-women-and-girls

Verkuyten, M., \& Hagendoom, L. (1998). Prejudice and self-categorization: The variable role of authoritarianism and in-group stereotypes. Personality and Social Psychology Bulletin, 21(1), 99-110.

Viki, T., Abrams, D., Viki, G. T., \& Abrams, D. (2002). But She Was Unfaithful : Benevolent Sexism and Reactions to Rape Victims Who Violate Traditional Gender Role Expectations But She Was Unfaithful : Benevolent Sexism and Reactions to Rape Victims Who Violate Traditional Gender Role Expectations, (January 2016). http://doi.org/10.1023/A

Wagner, U., van Dick, R., \& Zick, A. (2001). Sozialpsychologische analysen und erklärungen von fremdenfeindlichkeit in deutschland. Zeitschrift Für Sozialpsychologie, 32(2), 59-79. http://doi.org/10.1024//0044-3514.32.2.59

Wann, D. L. (1994). outgroup derogation when a valued social, 24(March 1992).

Whitley, B. E., \& Lee, S. E. (2000). The Relationship of Authoritarianism and Related 
Constructs to Attitudes Toward Homosexuality. Journal of Applied Social Psychology, 30(1), 144-170. http://doi.org/10.1111/j.1559-1816.2000.tb02309.x

Willard, N. E. (2007). Cyberbullying and Cyberthreats: Responding to the Challenge of Online Social Aggression, Threats, and Distress. Cyberbullying and cyberthreats: Responding to the challenge of online social aggression, threats, and distress.

Ybarra, M. L., Ph, D., Boyd, D., Ph, D., Korchmaros, J. D., Ph, D., .. A, M. (2012). Defining and measuring cyberbullying within the larger context of bullying victimization. Journal of Adolescent Health, 51(1), 53-58. http://doi.org/10.1016/j.jadohealth.2011.12.031

Yee, N. (2017). Beyond 50/50: Breaking Down The Percentage of Female Gamers by Genre. Retrieved from http://quanticfoundry.com/2017/01/19/female-gamers-by-genre/ 